\title{
CONTROL SYSTEM DEPENDING ON A PARAMETER ${ }^{1}$
}

\author{
Vladimir N. Ushakov ${ }^{\dagger}$, Aleksandr A. Ershov ${ }^{\dagger \dagger}$, Andrey V. Ushakov ${ }^{\dagger \dagger}$, \\ Oleg A. Kuvshinov ${ }^{\dagger \dagger \dagger}$ \\ Krasovskii Institute of Mathematics and Mechanics, \\ Ural Branch of the Russian Academy of Sciences, \\ 16 S. Kovalevskaya Str., Ekaterinburg, 620108, Russia \\ †ushak@imm.uran.ru, ††ale10919@yandex.ru, †† aushakov.pk@gmail.com, \\ ††††okuvshinov@inbox.ru
}

\begin{abstract}
A nonlinear control system depending on a parameter is considered in a finite-dimensional Euclidean space and on a finite time interval. The dependence on the parameter of the reachable sets and integral funnels of the corresponding differential inclusion system is studied. Under certain conditions on the control system, the degree of this dependence on the parameter is estimated. Problems of targeting integral funnels to a target set in the presence of an obstacle in strict and soft settings are considered. An algorithm for the numerical solution of this problem in the soft setting has been developed. An estimate of the error of the developed algorithm is obtained. An example of solving a specific problem for a control system in a two-dimensional phase space is given.
\end{abstract}

Keywords: Control system, Differential inclusion, Reachable set, Integral funnel, Parameter dependence, Approximation.

\section{Introduction}

A nonlinear control system depending on a parameter is considered in a finite-dimensional Euclidean space and on a finite time interval.

The reachable sets and integral funnels of the differential inclusion corresponding to the system are studied. The problems related to the study of reachable sets and integral funnels of dynamical systems are closely intertwined with numerous problems in the theory of dynamical systems including those that arise in control theory and the theory of differential games [5, 6, 10-13, 16, 17]. Various theoretical approaches and associated computational methods [1-3, 5-14, 16-21] are used in the study of reachable sets, their construction, and estimation. These control problems and differential games include, for example, various types of approach problems, resolving constructions of which include one of the main components that are called solvability sets, i.e., the sets of those positions of the control system from which the approach problem is solvable [10-13]. For many problems, these sets can be described quite simply in terms of reachable sets and integral funnels $[1,2,5-9,12,13,16-21]$. Some problems can be formulated as problems of the theory of controllability of dynamical systems [19].

In this paper, we study the dependence on a parameter of reachable sets and integral funnels: the degree of this dependence on the parameter is estimated under certain conditions imposed on the control system. We introduce systems of sets in the phase space that approximate reachable sets and integral funnels on a given time interval corresponding to a finite partition of this interval. In this case, the degree of dependence on the parameter of the approximating system of sets is first estimated, and then this estimate is used to estimate the dependence on the parameter of

\footnotetext{
${ }^{1}$ This research was supported by the Russian Science Foundation (project no. 19-11-00105).
} 
the reachable sets and integral funnels of the differential inclusion. This approach is natural and especially useful for studying specific applied control problems, when, in the end, one has to deal not with ideal reachable sets and integral funnels, but with their approximations corresponding to a discrete representation of the time interval.

\section{Estimates of reachable sets and integral funnels of differential inclusions}

Consider a control system $\Sigma$

$$
\frac{d x}{d t}=f_{\alpha}(t, x, u), \quad u \in P \in \operatorname{comp}\left(\mathbb{R}^{p}\right)
$$

on a time interval $\left[t_{0}, \vartheta\right], t_{0}<\vartheta<\infty$; here $x \in \mathbb{R}^{n}$ is the phase vector of $\Sigma, u$ is the control vector, $\alpha$ is a parameter from a set $\mathscr{L} \in \operatorname{comp}\left(\mathbb{R}^{l}\right) ; \operatorname{comp}\left(\mathbb{R}^{k}\right)$ is the set of compact subsets of $\mathbb{R}^{k}$ with the Hausdorff metric

$$
d\left(X^{(1)}, X^{(2)}\right)=\max \left(h\left(X^{(1)}, X^{(2)}\right), \quad h\left(X^{(2)}, X^{(1)}\right)\right), \quad h\left(X^{(1)}, X^{(2)}\right)=\max _{x^{(1)} \in X^{(1)}} \rho\left(x^{(1)}, X^{(2)}\right)
$$

is the Hausdorff deviation of $X^{(1)}$ from $X^{(2)}$, where

$$
\rho\left(x^{(1)}, X^{(2)}\right)=\min _{x^{(2)} \in X^{(2)}}\left\|x^{(1)}-x^{(2)}\right\| .
$$

We assume that the system $\Sigma$ satisfies the following conditions.

A. The function $f_{\alpha}(t, x, u)$ is defined on $\left[t_{0}, \vartheta\right] \times \mathbb{R}^{n} \times P \times \mathscr{L}$ and, for any bounded and closed domain $D \subset\left[t_{0}, \vartheta\right] \times \mathbb{R}^{n}$, there are a continuous function $\omega^{*}(r), r \in(0, \infty)\left(\omega^{*}(r) \downarrow 0, r \downarrow 0\right)$ and a continuous function $L(t) \in(0, \infty), t \in\left[t_{0}, \vartheta\right]$, satisfying the relations

$$
\begin{gathered}
\left\|f_{\alpha}(t, x, u)-f_{\beta}(\tau, x, u)\right\| \leqslant \omega^{*}(|t-\tau|+\|\alpha-\beta\|), \\
(t, x) \in D, \quad(\tau, x) \in D, \quad u \in P, \quad \alpha, \beta \in \mathscr{L} ; \\
\left\|f_{\alpha}(t, x, u)-f_{\alpha}(t, y, u)\right\| \leqslant L(t)\|x-y\|, \\
(t, x) \in D, \quad(t, y) \in D, \quad u \in P, \quad \alpha \in \mathscr{L} .
\end{gathered}
$$

B. There is $\gamma \in(0, \infty)$ such that

$$
\left\|f_{\alpha}(t, x, u)\right\| \leqslant \gamma(1+\|x\|), \quad(t, x, u) \in\left[t_{0}, \vartheta\right] \times \mathbb{R}^{n} \times P, \quad \alpha \in \mathscr{L} .
$$

We introduce a multivalued mapping

$$
\begin{gathered}
(t, x) \mapsto F_{\alpha}(t, x)=\operatorname{co} \mathcal{F}_{\alpha}(t, x), \\
\mathcal{F}_{\alpha}(t, x)=\left\{f_{\alpha}(t, x, u): u \in P\right\} \in \operatorname{comp}\left(\mathbb{R}^{n}\right), \\
(t, x) \in\left[t_{0}, \vartheta\right] \times \mathbb{R}^{n}, \quad \alpha \in \mathscr{L} .
\end{gathered}
$$

The mapping $(t, x) \mapsto F_{\alpha}(t, x) \in \operatorname{comp}\left(\mathbb{R}^{n}\right)$ satisfies the following conditions.

$\mathbf{A}^{*}$. For any bounded and closed domain $D \subset\left[t_{0}, \vartheta\right] \times \mathbb{R}^{n}$, there are a continuous function $\omega^{*}(r)$, $r \in(0, \infty)\left(\omega^{*}(r) \downarrow 0, r \downarrow 0\right)$ and a continuous function $L(t) \in(0, \infty), t \in\left[t_{0}, \vartheta\right]$, satisfying the relations

$$
\begin{gathered}
d\left(F_{\alpha}(t, x), F_{\beta}(\tau, x)\right) \leqslant \omega^{*}(|t-\tau|+\|\alpha-\beta\|), \\
(t, x) \in D, \quad(\tau, x) \in D, \quad \alpha, \beta \in \mathscr{L} ; \\
d\left(F_{\alpha}(t, x), F_{\alpha}(t, y)\right) \leqslant L(t)\|x-y\|, \\
(t, x) \in D, \quad(t, y) \in D, \quad \alpha \in \mathscr{L} .
\end{gathered}
$$


$\mathbf{B}^{*}$. There is $\gamma \in(0, \infty)$ such that

$$
h\left(F_{\alpha}(t, x),\{\mathbf{0}\}\right) \leqslant \gamma \cdot(1+\|x\|), \quad(t, x, \alpha) \in\left[t_{0}, \vartheta\right] \times \mathbb{R}^{n} \times \mathscr{L} ;
$$

here $\mathbf{0}$ is the null-vector in $\mathbb{R}^{n}$.

Let us introduce on $\left[t_{0}, \vartheta\right]$ the differential inclusion

$$
\frac{d x}{d t} \in F_{\alpha}(t, x), \quad \alpha \in \mathscr{L}
$$

that satisfies the system $\Sigma$.

Let $t_{*}$ and $t^{*}\left(t_{*}<t^{*}\right)$ be from $\left[t_{0}, \vartheta\right], x_{*} \in \mathbb{R}^{n}, X_{*} \in \operatorname{comp}\left(\mathbb{R}^{n}\right)$, and $\alpha \in \mathscr{L}$.

Let us introduce the notation:

- $X_{\alpha}\left(t^{*}, t_{*}, x_{*}\right)$ is the reachable set of the differential inclusion (1.4) at the time $t^{*}$ with the initial point $x\left(t_{*}\right)=x_{*}$;

- $X_{\alpha}\left(t^{*}, t_{*}, X_{*}\right)=\bigcup_{x_{*} \in X_{*}} X_{\alpha}\left(t^{*}, t_{*}, x_{*}\right)$ is the reachable set of the differential inclusion (1.4) at the time $t^{*}$ with the initial set $X_{*}$.

It is known that $X_{\alpha}\left(t^{*}, t_{*}, X_{*}\right) \in \operatorname{comp}\left(\mathbb{R}^{n}\right)$, the mapping $\left(t^{*}, t_{*}, X_{*}\right) \mapsto X_{\alpha}\left(t^{*}, t_{*}, X_{*}\right)$ is continuous in $t^{*}$ on $\left[t_{*}, \vartheta\right]$ for fixed $\left(t_{*}, X_{*}\right) \in\left[t_{0}, \vartheta\right] \times \operatorname{comp}\left(\mathbb{R}^{n}\right)$ in the Hausdorff metric, and also $X_{\alpha}\left(t^{*}, t_{*}, X_{*}\right)$ continuously depends on $X_{*}$ for fixed $t_{*}, t^{*}$, and $\alpha$.

The mapping $\alpha \mapsto X_{\alpha}\left(t^{*}, t_{*}, X_{*}\right)$ is also continuous on $\mathscr{L}$ for fixed $\left(t^{*}, t_{*}, X_{*}\right), t_{0} \leqslant t_{*}<t^{*} \leqslant \vartheta$, and $X_{*} \in \operatorname{comp}\left(\mathbb{R}^{n}\right)$.

Let us refine the continuous dependence of $\alpha \mapsto X_{\alpha}\left(t^{*}, t_{*}, X_{*}\right)$ on the set $\mathscr{L}$. To do this, we derive an upper bound for the Hausdorff distance

$$
d\left(X_{\alpha}\left(t^{*}, t_{*}, X_{*}\right), X_{\beta}\left(t^{*}, t_{*}, X_{*}\right)\right), \quad \alpha, \beta \in \mathscr{L},
$$

which we represent as a function of $\|\alpha-\beta\|$.

It is known that, under the conditions $\mathbf{A}^{*}$ and $\mathbf{B}^{*}$, the reachable set $X_{\alpha}\left(t^{*}, t_{*}, X_{*}\right)$ satisfies the equality

$$
X_{\alpha}\left(t^{*}, t_{*}, X_{*}\right)=\lim _{\Delta=\Delta(\Gamma) \downarrow 0} \tilde{X}_{\alpha}^{\Gamma^{*}}\left(t^{*}\right) .
$$

Here $\widetilde{X}_{\alpha}^{\Gamma_{*}}\left(t^{*}\right) \subset \mathbb{R}^{n}, \alpha \in \mathscr{L}$ are the sets corresponding to the partition

$$
\begin{gathered}
\Gamma_{*}=\left\{\tau_{0}=t_{*}, \tau_{1}, \ldots, \tau_{i}, \ldots, \tau_{N}=t^{*}\right\} \\
\left(\tau_{i+1}-\tau_{i}=\Delta=\Delta\left(\Gamma_{*}\right)=N^{-1}\left(t^{*}-t_{*}\right), \quad i=\overline{0, N-1}\right)
\end{gathered}
$$

of the interval $\left[t_{*}, t^{*}\right]$ defined by the equality $\widetilde{X}_{\alpha}^{\Gamma_{*}}\left(t^{*}\right)=\widetilde{X}_{\alpha}^{\Gamma_{*}}\left(t_{N}\right)$ and the recurrence relations

$$
\widetilde{X}_{\alpha}^{\Gamma_{*}}\left(\tau_{0}\right)=X_{*}, \quad \widetilde{X}_{\alpha}^{\Gamma_{*}}\left(\tau_{i+1}\right)=\widetilde{X}_{\alpha}\left(\tau_{i+1}, \tau_{i}, \widetilde{X}_{\alpha}^{\Gamma_{*}}\left(\tau_{i}\right)\right), \quad i=\overline{0, N-1},
$$

where

$$
\begin{gathered}
\widetilde{X}_{\alpha}\left(\tau^{*}, \tau_{*}, W_{*}\right)=\left\{x^{*} \in \mathbb{R}^{n}: x^{*}=w_{*}+\left(\tau^{*}-\tau_{*}\right) f_{*}, w_{*} \in W_{*}, f_{*} \in F_{\alpha}\left(\tau_{*}, w_{*}\right)\right\}, \\
t_{*} \leqslant \tau_{*}<\tau^{*} \leqslant t^{*}, \quad W_{*} \in \operatorname{comp}\left(\mathbb{R}^{n}\right) .
\end{gathered}
$$

Taking into account the condition $\mathbf{B}^{*}$ and the size of the compact set $X_{*}$, we can specify a bounded and closed domain $D \subset\left[t_{0}, \vartheta\right] \times \mathbb{R}^{n}$ containing all sets arising in the subsequent reasoning 
and estimates in the space $\left[t_{0}, \vartheta\right] \times \mathbb{R}^{n}$. We assume that functions $\omega^{*}(r), r \in(0, \infty)$, and $L(t)$, $t \in\left[t_{0}, \vartheta\right]$, corresponding to this domain $D$ are used in further estimates.

We first estimate quantity (1.5) for a one-point set $X_{*}=\left\{x_{*}\right\},\left(t_{*}, x_{*}\right) \in D$.

When deriving an estimate for quantity (1.6), we will apply the so-called "step-by-step" reasoning scheme and "step-by-step" estimates, that is, we will move through the steps $\left[\tau_{i}, \tau_{i+1}\right]$, $i=\overline{0, N-1}$, of the partition $\Gamma_{*}$.

We start deriving an estimate with the interval $\left[\tau_{0}, \tau_{1}\right]$ of the partition $\Gamma_{*}$. Let us find an upper bound for the Hausdorff deviation

$$
h\left(\widetilde{X}_{\alpha}^{\Gamma_{*}}\left(\tau_{1}\right), \widetilde{X}_{\beta}^{\Gamma_{*}}\left(\tau_{1}\right)\right), \quad \alpha, \beta \in \mathscr{L}
$$

here $\widetilde{X}_{\alpha}^{\Gamma_{*}}\left(\tau_{1}\right)=\widetilde{X}_{\alpha}\left(\tau_{1}, \tau_{0}, x_{*}\right)$ and $\widetilde{X}_{\beta}^{\Gamma_{*}}\left(\tau_{1}\right)=\widetilde{X}_{\beta}\left(\tau_{1}, \tau_{0}, x_{*}\right)$.

In $\widetilde{X}_{\alpha}^{\Gamma_{*}}\left(\tau_{1}\right)$, we choose a point $x\left(\tau_{1}\right)$ such that $\rho\left(x\left(\tau_{1}\right), \widetilde{X}_{\beta}^{\Gamma_{*}}\left(\tau_{1}\right)\right)=h\left(\widetilde{X}_{\alpha}^{\Gamma_{*}}\left(\tau_{1}\right), \widetilde{X}_{\beta}^{\Gamma_{*}}\left(\tau_{1}\right)\right)$. The point $x\left(\tau_{1}\right)$ is representable as

$$
x\left(\tau_{1}\right)=x_{*}+\Delta f_{\alpha}\left(\tau_{0}\right), \quad f_{\alpha}\left(\tau_{0}\right) \in F_{\alpha}\left(\tau_{0}, x_{*}\right) .
$$

Let us choose a vector $f_{\beta}\left(\tau_{0}\right)$ in $F_{\beta}\left(\tau_{0}, x_{*}\right)$ closest to $f_{\alpha}\left(\tau_{0}\right)$. The following estimate is valid:

$$
\left\|f_{\alpha}\left(\tau_{0}\right)-f_{\beta}\left(\tau_{0}\right)\right\|=\rho\left(f_{\alpha}\left(\tau_{0}\right), F_{\beta}\left(\tau_{0}, x_{*}\right)\right) \leqslant h\left(F_{\alpha}\left(\tau_{0}, x_{*}\right), F_{\beta}\left(\tau_{0}, x_{*}\right)\right) \leqslant \omega^{*}(\|\alpha-\beta\|) .
$$

In $\widetilde{X}_{\beta}^{\Gamma_{*}}\left(\tau_{1}\right)$, we consider the point $y\left(\tau_{1}\right)=x_{*}+\Delta f_{\beta}\left(\tau_{0}\right), \Delta=\Delta\left(\Gamma_{*}\right)$. There is an estimate

$$
\left\|x\left(\tau_{1}\right)-y\left(\tau_{1}\right)\right\| \leqslant \Delta \omega^{*}(\|\alpha-\beta\|) .
$$

The definition of the point $x\left(\tau_{1}\right)$ and the inclusion $y\left(\tau_{1}\right) \in \tilde{X}_{\beta}^{\Gamma_{*}}\left(\tau_{1}\right)$ imply the estimate

$$
h\left(\tau_{1}\right) \leqslant \Delta \omega^{*}(\|\alpha-\beta\|) ;
$$

here $h\left(\tau_{1}\right)=h\left(\widetilde{X}_{\alpha}^{\Gamma_{*}}\left(\tau_{1}\right), \widetilde{X}_{\beta}^{\Gamma_{*}}\left(\tau_{1}\right)\right)$.

Let us turn to the next interval $\left[\tau_{1}, \tau_{2}\right]$ of the partition $\Gamma_{*}$ and consider the sets $\widetilde{X}_{\alpha}^{\Gamma_{*}}\left(\tau_{2}\right)=\widetilde{X}_{\alpha}\left(\tau_{2}, \tau_{1}, \widetilde{X}_{\alpha}^{\Gamma_{*}}\left(\tau_{1}\right)\right)$ and $\widetilde{X}_{\beta}^{\Gamma_{*}}\left(\tau_{2}\right)=\widetilde{X}_{\beta}\left(\tau_{2}, \tau_{1}, \widetilde{X}_{\beta}^{\Gamma_{*}}\left(\tau_{1}\right)\right)$.

In $\widetilde{X}_{\alpha}^{\Gamma_{*}}\left(\tau_{2}\right)$, we choose a point $x\left(\tau_{2}\right)$ such that

$$
\rho\left(x\left(\tau_{2}\right), \widetilde{X}_{\beta}^{\Gamma^{*}}\left(\tau_{2}\right)\right)=h\left(\widetilde{X}_{\alpha}^{\Gamma_{*}}\left(\tau_{2}\right), \widetilde{X}_{\beta}^{\Gamma_{*}}\left(\tau_{2}\right)\right) .
$$

The point $x\left(\tau_{2}\right)$ is representable as

$$
x\left(\tau_{2}\right)=x_{*}\left(\tau_{1}\right)+\Delta f_{\alpha}\left(\tau_{1}\right), \quad x_{*}\left(\tau_{1}\right) \in \widetilde{X}_{\alpha}^{\Gamma_{*}}\left(\tau_{1}\right), \quad f_{\alpha}\left(\tau_{1}\right) \in F_{\alpha}\left(\tau_{1}, x_{*}\left(\tau_{1}\right)\right) .
$$

Let us choose a point $y_{*}\left(\tau_{1}\right)$ in $\widetilde{X}_{\beta}^{\Gamma_{*}}\left(\tau_{1}\right)$ closest to $x_{*}\left(\tau_{1}\right)$ :

$$
\left\|x_{*}\left(\tau_{1}\right)-y_{*}\left(\tau_{1}\right)\right\|=\rho\left(x_{*}\left(\tau_{1}\right), \tilde{X}_{\beta}^{\Gamma_{*}}\left(\tau_{1}\right)\right) .
$$

The following estimate is valid:

$$
\left\|x_{*}\left(\tau_{1}\right)-y_{*}\left(\tau_{1}\right)\right\| \leqslant h\left(\tau_{1}\right) .
$$

Let us choose a vector $f_{\beta}\left(\tau_{1}\right)$ in $F_{\beta}\left(\tau_{1}, y_{*}\left(\tau_{1}\right)\right)$ closest to $f_{\alpha}\left(\tau_{1}\right)$. By (1.2) and (1.3), the following inequalities hold:

$$
\begin{gathered}
\left\|f_{\alpha}\left(\tau_{1}\right)-f_{\beta}\left(\tau_{1}\right)\right\| \leqslant h\left(F_{\alpha}\left(\tau_{1}, x_{*}\left(\tau_{1}\right)\right), F_{\beta}\left(\tau_{1}, y_{*}\left(\tau_{1}\right)\right)\right) \\
\leqslant d\left(F_{\alpha}\left(\tau_{1}, x_{*}\left(\tau_{1}\right)\right), F_{\beta}\left(\tau_{1}, y_{*}\left(\tau_{1}\right)\right)\right) \leqslant \omega^{*}(\|\alpha-\beta\|)+L\left(\tau_{1}\right) h\left(\tau_{1}\right) .
\end{gathered}
$$


We introduce the point

$$
y\left(\tau_{2}\right)=y_{*}\left(\tau_{1}\right)+\Delta f_{\beta}\left(\tau_{1}\right), \quad y_{*}\left(\tau_{1}\right) \in \tilde{X}_{\beta}^{\Gamma_{*}}\left(\tau_{1}\right), \quad f_{\beta}\left(\tau_{1}\right) \in F_{\beta}\left(\tau_{1}, y_{*}\left(\tau_{1}\right)\right) .
$$

The points $x\left(\tau_{2}\right)$ and $y\left(\tau_{2}\right)$ satisfy the inequalities

$$
\begin{gathered}
\left\|x\left(\tau_{2}\right)-y\left(\tau_{2}\right)\right\| \leqslant\left\|x_{*}\left(\tau_{1}\right)-y_{*}\left(\tau_{1}\right)\right\|+\Delta\left\|f_{\alpha}\left(\tau_{1}\right)-f_{\beta}\left(\tau_{1}\right)\right\| \\
\leqslant h\left(\tau_{1}\right)+\Delta \cdot\left(\omega^{*}(\|\alpha-\beta\|)+L\left(\tau_{1}\right) h\left(\tau_{1}\right)\right) \\
\leqslant \Delta \omega^{*}(\|\alpha-\beta\|)+e^{L\left(\tau_{1}\right) \Delta_{1}} \cdot h\left(\tau_{1}\right),
\end{gathered}
$$

where $\Delta_{1}=\Delta=\Delta\left(\Gamma_{*}\right)$.

Considering (1.8) and the inclusion $y\left(\tau_{2}\right) \in \widetilde{X}_{\beta}^{\Gamma_{*}}\left(\tau_{2}\right)=\widetilde{X}_{\beta}\left(\tau_{2}, \tau_{1}, \widetilde{X}_{\beta}^{\Gamma_{*}}\left(\tau_{1}\right)\right)$, we obtain

$$
h\left(\widetilde{X}_{\alpha}^{\Gamma_{*}}\left(\tau_{2}\right), \widetilde{X}_{\beta}^{\Gamma_{*}}\left(\tau_{2}\right)\right) \leqslant\left\|x\left(\tau_{2}\right)-y\left(\tau_{2}\right)\right\| .
$$

Estimates (1.9) and (1.10) imply that

$$
h\left(\widetilde{X}_{\alpha}^{\Gamma_{*}}\left(\tau_{2}\right), \widetilde{X}_{\beta}^{\Gamma^{*}}\left(\tau_{2}\right)\right) \leqslant \Delta \omega^{*}(\|\alpha-\beta\|)+e^{L\left(\tau_{1}\right) \Delta_{1}} h\left(\tau_{1}\right) .
$$

Consider the next interval $\left[\tau_{2}, \tau_{3}\right]$ of the partition $\Gamma_{*}$ and the sets $\widetilde{X}_{\alpha}^{\Gamma_{*}}\left(\tau_{3}\right)=\widetilde{X}_{\alpha}\left(\tau_{3}, \tau_{2}, \widetilde{X}_{\alpha}^{\Gamma_{*}}\left(\tau_{2}\right)\right)$ and $\widetilde{X}_{\beta}^{\Gamma *}\left(\tau_{3}\right)=\widetilde{X}_{\beta}\left(\tau_{3}, \tau_{2}, \widetilde{X}_{\beta}^{\Gamma *}\left(\tau_{2}\right)\right)$.

Let us find an upper bound for the Hausdorff deviation

$$
h\left(\widetilde{X}_{\alpha}^{\Gamma^{*}}\left(\tau_{3}\right), \widetilde{X}_{\beta}^{\Gamma^{*}}\left(\tau_{3}\right)\right), \quad \alpha, \beta \in \mathscr{L} .
$$

To do this, we choose a point $x\left(\tau_{3}\right)$ in the $\widetilde{X}_{\alpha}^{\Gamma_{*}}\left(\tau_{3}\right)$ such that

$$
\rho\left(x\left(\tau_{3}\right), \widetilde{X}_{\beta}^{\Gamma_{*}}\left(\tau_{3}\right)\right)=h\left(\widetilde{X}_{\alpha}^{\Gamma_{*}}\left(\tau_{3}\right), \widetilde{X}_{\beta}^{\Gamma_{*}}\left(\tau_{3}\right)\right) .
$$

The point $x\left(\tau_{3}\right)$ is representable as

$$
x\left(\tau_{3}\right)=x_{*}\left(\tau_{2}\right)+\Delta f_{\alpha}\left(\tau_{2}\right), \quad x_{*}\left(\tau_{2}\right) \in \widetilde{X}_{\alpha}^{\Gamma_{*}}\left(\tau_{2}\right), \quad f_{\alpha}\left(\tau_{2}\right) \in F_{\alpha}\left(\tau_{2}, x_{*}\left(\tau_{2}\right)\right) .
$$

Let us choose a point $y_{*}\left(\tau_{2}\right)$ in $\widetilde{X}_{\beta}^{\Gamma_{*}}\left(\tau_{2}\right)$ closest to the point $x_{*}\left(\tau_{2}\right)$ :

$$
\left\|x_{*}\left(\tau_{2}\right)-y_{*}\left(\tau_{2}\right)\right\|=\rho\left(x_{*}\left(\tau_{2}\right), \widetilde{X}_{\beta}^{\Gamma_{*}}\left(\tau_{2}\right)\right) .
$$

The following inequality is valid

$$
\left\|x_{*}\left(\tau_{2}\right)-y_{*}\left(\tau_{2}\right)\right\| \leqslant h\left(\widetilde{X}_{\alpha}^{\Gamma_{*}}\left(\tau_{2}\right), \widetilde{X}_{\beta}^{\Gamma_{*}}\left(\tau_{2}\right)\right) .
$$

Let us choose a vector $f_{\beta}\left(\tau_{2}\right)$ in $F_{\beta}\left(\tau_{2}, y_{*}\left(\tau_{2}\right)\right)$ closest to $f_{\alpha}\left(\tau_{2}\right)$. We obtain the estimate

$$
\begin{gathered}
\left\|f_{\alpha}\left(\tau_{2}\right)-f_{\beta}\left(\tau_{2}\right)\right\| \leqslant h\left(F_{\alpha}\left(\tau_{2}, x_{*}\left(\tau_{2}\right)\right), F_{\beta}\left(\tau_{2}, y_{*}\left(\tau_{2}\right)\right)\right) \\
\leqslant d\left(F_{\alpha}\left(\tau_{2}, x_{*}\left(\tau_{2}\right)\right), F_{\beta}\left(\tau_{2}, y_{*}\left(\tau_{2}\right)\right)\right) \\
\leqslant \omega^{*}(\|\alpha-\beta\|)+L\left(\tau_{2}\right)\left\|x_{*}\left(\tau_{2}\right)-y_{*}\left(\tau_{2}\right)\right\| .
\end{gathered}
$$

Consider the point $y\left(\tau_{3}\right)=y_{*}\left(\tau_{2}\right)+\Delta f_{\beta}\left(\tau_{2}\right)$ in $\widetilde{X}_{\beta}^{\Gamma_{*}}\left(\tau_{3}\right)$. The points $x\left(\tau_{3}\right)$ and $y\left(\tau_{3}\right)$ satisfy the inequalities

$$
\begin{gathered}
\left\|x\left(\tau_{3}\right)-y\left(\tau_{3}\right)\right\| \leqslant\left\|x_{*}\left(\tau_{2}\right)-y_{*}\left(\tau_{2}\right)\right\|+\Delta \cdot\left(\omega^{*}(\|\alpha-\beta\|)+L\left(\tau_{2}\right)\left\|x_{*}\left(\tau_{2}\right)-y_{*}\left(\tau_{2}\right)\right\|\right) \\
\leqslant \Delta \cdot \omega^{*}(\|\alpha-\beta\|)+e^{L\left(\tau_{2}\right) \Delta_{2}} h\left(\widetilde{X}_{\alpha}^{\Gamma_{*}}\left(\tau_{2}\right), \widetilde{X}_{\beta}^{\Gamma_{*}}\left(\tau_{2}\right)\right) \\
\leqslant \Delta \cdot \omega^{*}(\|\alpha-\beta\|)+e^{L\left(\tau_{2}\right) \Delta_{2}}\left(\Delta \cdot \omega^{*}(\|\alpha-\beta\|)+e^{L\left(\tau_{1}\right) \Delta_{1}} h\left(\tau_{1}\right)\right),
\end{gathered}
$$


where $\Delta_{2}=\Delta=\Delta\left(\Gamma_{*}\right)$.

As a result, we get

$$
\left\|x\left(\tau_{3}\right)-y\left(\tau_{3}\right)\right\| \leqslant\left(1+e^{L\left(\tau_{2}\right) \Delta_{2}}\right) \cdot \Delta \omega^{*}(\|\alpha-\beta\|)+e^{L\left(\tau_{1}\right) \Delta_{1}+L\left(\tau_{2}\right) \Delta_{2}} \cdot h\left(\tau_{1}\right) .
$$

Considering (1.12) and the inclusion $y\left(\tau_{3}\right) \in \widetilde{X}_{\beta}^{\Gamma^{*}}\left(\tau_{3}\right)$, we get

$$
h\left(\widetilde{X}_{\alpha}^{\Gamma_{*}}\left(\tau_{3}\right), \widetilde{X}_{\beta}^{\Gamma^{*}}\left(\tau_{3}\right)\right) \leqslant\left\|x\left(\tau_{3}\right)-y\left(\tau_{3}\right)\right\| .
$$

From (1.13) and (1.14), it follows that

$$
h\left(\widetilde{X}_{\alpha}^{\Gamma_{*}}\left(\tau_{3}\right), \widetilde{X}_{\beta}^{\Gamma_{*}}\left(\tau_{3}\right)\right) \leqslant\left(1+e^{L\left(\tau_{2}\right) \Delta_{2}}\right) \cdot \Delta \omega^{*}(\|\alpha-\beta\|)+e^{L\left(\tau_{1}\right) \Delta_{1}+L\left(\tau_{2}\right) \Delta_{2}} \cdot h\left(\tau_{1}\right) .
$$

For a final understanding of the structure of the estimate of the quantity $h\left(\widetilde{X}_{\alpha}^{\Gamma_{*}}\left(\tau_{i+1}\right), \widetilde{X}_{\beta}^{\Gamma_{*}}\left(\tau_{i+1}\right)\right)$, $i=\overline{0, N-1}$, we consider the next interval $\left[\tau_{3}, \tau_{4}\right]$ of the partition $\Gamma_{*}$ and the sets

$$
\tilde{X}_{\alpha}^{\Gamma_{*}}\left(\tau_{4}\right)=\tilde{X}_{\alpha}\left(\tau_{4}, \tau_{3}, \tilde{X}_{\alpha}^{\Gamma_{*}}\left(\tau_{3}\right)\right) \quad \text { and } \quad \tilde{X}_{\beta}^{\Gamma_{*}}\left(\tau_{4}\right)=\tilde{X}_{\beta}\left(\tau_{4}, \tau_{3}, \widetilde{X}_{\beta}^{\Gamma_{*}}\left(\tau_{3}\right)\right) .
$$

Let us estimate from above the quantity

$$
h\left(\widetilde{X}_{\alpha}^{\Gamma_{*}}\left(\tau_{4}\right), \widetilde{X}_{\beta}^{\Gamma_{*}}\left(\tau_{4}\right)\right), \quad \alpha, \beta \in \mathscr{L} .
$$

To do this, we choose a point $x\left(\tau_{4}\right)$ in $\widetilde{X}_{\alpha}^{\Gamma_{*}}\left(\tau_{4}\right)$ such that

$$
\rho\left(x\left(\tau_{4}\right), \widetilde{X}_{\beta}^{\Gamma_{*}}\left(\tau_{4}\right)\right)=h\left(\widetilde{X}_{\alpha}^{\Gamma_{*}}\left(\tau_{4}\right), \widetilde{X}_{\beta}^{\Gamma_{*}}\left(\tau_{4}\right)\right) .
$$

The point $x\left(\tau_{4}\right)$ is representable in the form

$$
x\left(\tau_{4}\right)=x_{*}\left(\tau_{3}\right)+\Delta f_{\alpha}\left(\tau_{3}\right), \quad x_{*}\left(\tau_{3}\right) \in \tilde{X}_{\alpha}^{\Gamma_{*}}\left(\tau_{3}\right), \quad f_{\alpha}\left(\tau_{3}\right) \in F_{\alpha}\left(\tau_{3}, x_{*}\left(\tau_{3}\right)\right) .
$$

Let us choose a point $y_{*}\left(\tau_{3}\right)$ in $\widetilde{X}_{\beta}^{\Gamma_{*}}\left(\tau_{3}\right)$ closest to $x_{*}\left(\tau_{3}\right)$ :

$$
\left\|x_{*}\left(\tau_{3}\right)-y_{*}\left(\tau_{3}\right)\right\|=\rho\left(x_{*}\left(\tau_{3}\right), \widetilde{X}_{\beta}^{\Gamma_{*}}\left(\tau_{3}\right)\right) .
$$

The following inequality holds:

$$
\left\|x_{*}\left(\tau_{3}\right)-y_{*}\left(\tau_{3}\right)\right\| \leqslant h\left(\widetilde{X}_{\alpha}^{\Gamma_{*}}\left(\tau_{3}\right), \widetilde{X}_{\beta}^{\Gamma_{*}}\left(\tau_{3}\right)\right) .
$$

Let us choose a vector $f_{\beta}\left(\tau_{3}\right)$ in $F_{\beta}\left(\tau_{3}, y_{*}\left(\tau_{3}\right)\right)$ closest to $f_{\alpha}\left(\tau_{3}\right)$.

By relations (1.2) and (1.3), the following estimate is valid:

$$
\begin{gathered}
\left\|f_{\alpha}\left(\tau_{3}\right)-f_{\beta}\left(\tau_{3}\right)\right\| \leqslant h\left(F_{\alpha}\left(\tau_{3}, x_{*}\left(\tau_{3}\right)\right), F_{\beta}\left(\tau_{3}, y_{*}\left(\tau_{3}\right)\right)\right) \leqslant d\left(F_{\alpha}\left(\tau_{3}, x_{*}\left(\tau_{3}\right)\right), F_{\beta}\left(\tau_{3}, y_{*}\left(\tau_{3}\right)\right)\right) \\
\leqslant \omega^{*}(\|\alpha-\beta\|)+L\left(\tau_{3}\right)\left\|x_{*}\left(\tau_{3}\right)-y_{*}\left(\tau_{3}\right)\right\| .
\end{gathered}
$$

Let us choose the point $y\left(\tau_{4}\right)=y_{*}\left(\tau_{3}\right)+\Delta f_{\beta}\left(\tau_{3}\right)$ in $\tilde{X}_{\beta}^{\Gamma_{*}}\left(\tau_{4}\right)$.

Taking into account (1.15) and (1.16), we obtain

$$
\begin{gathered}
\left\|x\left(\tau_{4}\right)-y\left(\tau_{4}\right)\right\| \leqslant\left\|x_{*}\left(\tau_{3}\right)-y_{*}\left(\tau_{3}\right)\right\|+\Delta\left\|f_{\alpha}\left(\tau_{3}\right)-f_{\beta}\left(\tau_{3}\right)\right\| \\
\leqslant\left\|x_{*}\left(\tau_{3}\right)-y_{*}\left(\tau_{3}\right)\right\|+\Delta \omega^{*}(\|\alpha-\beta\|)+L\left(\tau_{3}\right)\left\|x_{*}\left(\tau_{3}\right)-y_{*}\left(\tau_{3}\right)\right\| \leqslant \\
\leqslant \Delta \omega^{*}(\|\alpha-\beta\|)+e^{L\left(\tau_{3}\right) \Delta_{3}}\left(\left(1+e^{L\left(\tau_{2}\right) \Delta_{2}}\right) \cdot \Delta \omega^{*}(\|\alpha-\beta\|)+e^{L\left(\tau_{1}\right) \Delta_{1}+L\left(\tau_{2}\right) \Delta_{2}} \cdot h\left(\tau_{1}\right)\right), \\
\Delta_{3}=\Delta=\Delta\left(\Gamma_{*}\right) .
\end{gathered}
$$


As a result, we get the estimate

$$
\begin{aligned}
\left\|x\left(\tau_{4}\right)-y\left(\tau_{4}\right)\right\| \leqslant & \Delta \omega^{*}(\|\alpha-\beta\|) \cdot\left(1+e^{L\left(\tau_{3}\right) \Delta_{3}}+e^{L\left(\tau_{3}\right) \Delta_{3}+L\left(\tau_{2}\right) \Delta_{2}}\right) \\
& +e^{L\left(\tau_{3}\right) \Delta_{3}+L\left(\tau_{2}\right) \Delta_{2}+L\left(\tau_{1}\right) \Delta_{1}} \cdot h\left(\tau_{1}\right) .
\end{aligned}
$$

Further, taking into account the choice of the points $x\left(\tau_{4}\right)$ and $y\left(\tau_{4}\right)$, we obtain

$$
h\left(\widetilde{X}_{\alpha}^{\Gamma_{*}}\left(\tau_{4}\right), \widetilde{X}_{\beta}^{\Gamma_{*}}\left(\tau_{4}\right)\right) \leqslant\left\|x\left(\tau_{4}\right)-y\left(\tau_{4}\right)\right\| .
$$

The latter two inequalities imply the estimate

$$
\begin{aligned}
h\left(\widetilde{X}_{\alpha}^{\Gamma_{*}}\left(\tau_{4}\right), \widetilde{X}_{\beta}^{\Gamma_{*}^{*}}\left(\tau_{4}\right)\right) & \leqslant\left(1+e^{L\left(\tau_{3}\right) \Delta_{3}}+e^{L\left(\tau_{3}\right) \Delta_{3}+L\left(\tau_{2}\right) \Delta_{2}}\right) \cdot \Delta \omega^{*}(\|\alpha-\beta\|)+ \\
& +e^{L\left(\tau_{3}\right) \Delta_{3}+L\left(\tau_{2}\right) \Delta_{2}+L\left(\tau_{1}\right) \Delta_{1}} \cdot h\left(\tau_{1}\right) .
\end{aligned}
$$

Analyzing estimates (1.11), (1.15), and (1.17), we conclude that the interval $\left[\tau_{i}, \tau_{i+1}\right]$, $i=\overline{1, N-1}$, of the partition $\Gamma_{*}$ corresponds to the following estimate of the Hausdorff deviation $h\left(\widetilde{X}_{\alpha}^{\Gamma_{*}}\left(\tau_{i+1}\right), \widetilde{X}_{\beta}^{\Gamma_{*}}\left(\tau_{i+1}\right)\right)$ of the set $\widetilde{X}_{\alpha}^{\Gamma_{*}}\left(\tau_{i+1}\right)=\widetilde{X}_{\alpha}\left(\tau_{i+1}, \tau_{i}, \widetilde{X}_{\alpha}^{\Gamma_{*}}\left(\tau_{i}\right)\right)$ from the set $\widetilde{X}_{\beta}^{\Gamma_{*}}\left(\tau_{i+1}\right)=\widetilde{X}_{\beta}\left(\tau_{i+1}, \tau_{i}, \widetilde{X}_{\beta}^{\Gamma_{*}}\left(\tau_{i}\right)\right)$ :

$$
\begin{gathered}
h\left(\widetilde{X}_{\alpha}^{\Gamma_{*}}\left(\tau_{i+1}\right), \widetilde{X}_{\beta}^{\Gamma_{*}}\left(\tau_{i+1}\right)\right) \leqslant\left(1+e^{\sum_{k=i}^{i} L\left(\tau_{k}\right) \Delta_{k}}+e^{\sum_{k=i-1}^{i} L\left(\tau_{k}\right) \Delta_{k}}+\right. \\
\left.+e^{\sum_{k=i-2}^{i} L\left(\tau_{k}\right) \Delta_{k}}+\ldots+e^{\sum_{k=1}^{i} L\left(\tau_{k}\right) \Delta_{k}}\right) \cdot h\left(\tau_{1}\right) .
\end{gathered}
$$

Further, given that $h\left(\tau_{1}\right)=h\left(\widetilde{X}_{\alpha}^{\Gamma_{*}}\left(\tau_{1}\right), \widetilde{X}_{\beta}^{\Gamma^{*}}\left(\tau_{1}\right)\right)$ satisfies (1.7), from (1.18) we obtain the following estimate:

$$
\begin{gathered}
h\left(\widetilde{X}_{\alpha}^{\Gamma_{*}}\left(\tau_{i+1}\right), \widetilde{X}_{\beta}^{\Gamma_{*}}\left(\tau_{i+1}\right)\right) \leqslant\left(1+e^{\sum_{k=i}^{i} L\left(\tau_{k}\right) \Delta_{k}}+e^{\sum_{k=i-1}^{i} L\left(\tau_{k}\right) \Delta_{k}}+\right. \\
\left.+e^{\sum_{k=i-2}^{i} L\left(\tau_{k}\right) \Delta_{k}}+\ldots+e^{\sum_{k=1}^{i} L\left(\tau_{k}\right) \Delta_{k}}\right) \Delta \omega^{*}(\|\alpha-\beta\|) .
\end{gathered}
$$

Let us supplement the estimate (1.19) with a comment related to the function $L(t)$ continuous on the interval $\left[t_{0}, \vartheta\right]$, which was introduced in the condition $\mathbf{B}$.

Remark 1. In numerous studies devoted to nonlinear control systems described by ordinary differential equations, the condition of the local Lipschitz property of its right-hand side with respect to the phase variable is introduced as one of the main conditions imposed on the system. In this case, often in the process of studying control problems for such systems, it becomes necessary to choose in the space of positions of the control system a domain $D$ that would contain all the components of the resolving structure (resolving sets, trajectories of systems, phase constraints, etc.). In other words, quite often, when studying and solving control problems, it is necessary to choose a domain $D$ in the space of positions of the system, in which the problem is solved. In this case, the Lipschitz constant $L$ corresponding to this domain $D$ is used for constructing a solution and justifying its correctness. However, the introduced domain $D$ may turn out to be large, and the corresponding constant $L$ may also turn out to be large. In this case, the estimates justifying the correctness of the solution of the control problem in which this constant $L$ is involved may turn out to be rough. For various reasons, these estimates in a specific control problem (with a specific control system) may be unsatisfactory from the point of view of the person solving the problem and counting on finer estimates. In this regard, taking into consideration the conditions imposed on the nonlinear control system (1.1), in this paper, instead of the traditional local Lipschitz condition with the Lipschitz constant $L$, we introduce a continuous function $L(t) \in(0, \infty)$ on $\left[t_{0}, \vartheta\right]$, which is more suitable for the dynamics of (1.1). Estimate (1.19) of $h\left(\widetilde{X}_{\alpha}^{\Gamma_{*}}\left(\tau_{i+1}\right), \widetilde{X}_{\beta}^{\Gamma_{*}}\left(\tau_{i+1}\right)\right)$ is more accurate in the sense that, for each interval $\left[\tau_{i}, \tau_{i+1}\right]$ of the partition $\Gamma_{*}$, the step-by-step estimates involve 
its own value $L\left(\tau_{i}\right) \in(0, \infty)$, which is close to $L(t), t \in\left[\tau_{i}, \tau_{i+1}\right]$, for small $\Delta=\Delta\left(\Gamma_{*}\right)$, and not some constant $L \in(0, \infty)$ common to all $\left[\tau_{i}, \tau_{i+1}\right]$ from the interval $\left[t_{0}, \vartheta\right]$. Note, however, that this reasoning assumes that the domain $D$ is in the position space of the system and the corresponding function $L(t)$ on $\left[t_{0}, \vartheta\right]$ is chosen sufficiently adequately to the dynamics of the control system. So, for example, in control problems related to the study of reachable sets and integral funnels, the domain $D$ should track more or less accurately the dynamics of reachable sets and, therefore, the spatial structure of integral funnels.

Thus, in many specific control problems, the problem of choosing the domain $D$ and the corresponding function $L(t), t \in\left[t_{0}, \vartheta\right]$, in our opinion, is very significant, since the accuracy of the estimates related to solving problems depends on this.

Obviously, one of the ways to solve this problem in each specific task related to the study of reachable sets and integral funnels is to form the domain $D$ and the function $L(t), t \in\left[t_{0}, \vartheta\right]$, in a step-by-step procedure (by time layers $\left[\tau_{i}, \tau_{i+1}\right] \times \mathbb{R}^{n}, i=0,1, \ldots, N-1$ ) along with the construction of reachable sets.

Let us now return to estimate (1.19) and present some roughness of this estimate in a simpler form.

Replacing in (1.19) 1 and the exponents $e^{\sum_{k=r}^{i} L\left(\tau_{k}\right) \Delta_{k}}, r=\overline{1, i}$, by the exponent $e^{\sum_{k=0}^{i} L\left(\tau_{k}\right) \Delta_{k}}$, we get the estimate

$$
h\left(\widetilde{X}_{\alpha}^{\Gamma_{*}}\left(\tau_{i+1}\right), \widetilde{X}_{\beta}^{\Gamma_{*}}\left(\tau_{i+1}\right)\right) \leqslant e^{\sum_{k=0}^{i} L\left(\tau_{k}\right) \Delta_{k}} \cdot(i+1) \Delta \omega^{*}(\|\alpha-\beta\|)
$$

i.e.,

$$
h\left(\widetilde{X}_{\alpha}^{\Gamma_{*}}\left(\tau_{i+1}\right), \widetilde{X}_{\beta}^{\Gamma_{*}^{*}}\left(\tau_{i+1}\right)\right) \leqslant e^{\sum_{k=0}^{i} L\left(\tau_{k}\right) \Delta_{k}} \cdot\left(\tau_{i+1}-\tau_{0}\right) \omega^{*}(\|\alpha-\beta\|) .
$$

In particular, the following estimate holds:

$$
h\left(\widetilde{X}_{\alpha}^{\Gamma_{*}}\left(t^{*}\right), \widetilde{X}_{\beta}^{\Gamma_{*}}\left(t^{*}\right)\right) \leqslant e^{\sum_{k=0}^{N-1} L\left(\tau_{k}\right) \Delta_{k}} \cdot\left(t^{*}-t_{*}\right) \omega^{*}(\|\alpha-\beta\|) .
$$

Replacing in estimates (1.19)-(1.21) the numbers $L\left(\tau_{k}\right), k=\overline{0, N-1}$, with some $L$ satisfying the inequality $0<\max _{t \in\left[t_{0}, \vartheta\right]} L(t) \leqslant L<\infty$, we obtain the following estimates for $i \in \overline{1, N-1}$ and $\alpha$, $\beta$ from $\mathscr{L}$, respectively:

$$
\begin{gathered}
h\left(\widetilde{X}_{\alpha}^{\Gamma_{*}}\left(\tau_{i+1}\right), \widetilde{X}_{\beta}^{\Gamma_{*}}\left(\tau_{i+1}\right)\right) \leqslant \sum_{k=0}^{i} e^{L k \Delta} \Delta \omega^{*}(\|\alpha-\beta\|), \\
h\left(\widetilde{X}_{\alpha}^{\Gamma_{*}}\left(\tau_{i+1}\right), \widetilde{X}_{\beta}^{\Gamma^{*}}\left(\tau_{i+1}\right)\right) \leqslant e^{L \cdot\left(\tau_{i+1}-\tau_{0}\right)}\left(\tau_{i+1}-\tau_{0}\right) \omega^{*}(\|\alpha-\beta\|), \\
h\left(\widetilde{X}_{\alpha}^{\Gamma_{*}^{*}}\left(t^{*}\right), \widetilde{X}_{\beta}^{\Gamma^{*}}\left(t^{*}\right)\right) \leqslant e^{L \cdot\left(t^{*}-t_{*}\right)}\left(t^{*}-t_{*}\right) \omega^{*}(\|\alpha-\beta\|) .
\end{gathered}
$$

Reasoning similar to those given above for $h\left(\widetilde{X}_{\alpha}^{\Gamma_{*}}\left(\tau_{i+1}\right)\right.$ and $\left.\widetilde{X}_{\beta}^{\Gamma_{*}^{*}}\left(\tau_{i+1}\right)\right)$ yields estimates for $h\left(\widetilde{X}_{\beta}^{\Gamma_{*}}\left(\tau_{i+1}\right), \widetilde{X}_{\alpha}^{\Gamma_{*}}\left(\tau_{i+1}\right)\right)$ similar to (1.19)-(1.24). Taking this into account, we come to the following statement.

Lemma 1. Assume that $\left[t_{*}, t^{*}\right] \subset\left[t_{0}, \vartheta\right], X_{*} \in \operatorname{comp}\left(\mathbb{R}^{n}\right), \Gamma_{*}=\left\{\tau_{0}=t_{*}, \tau_{1}, \ldots, \tau_{i}, \ldots, \tau_{N}=t^{*}\right\}$ $\left(\tau_{i+1}-\tau_{i}=\Delta_{i}=\Delta, i=\overline{0, N-1}\right)$, and $\left\{\widetilde{X}_{\alpha}^{\Gamma_{*}}\left(\tau_{i}\right): \tau_{i} \in \Gamma_{*}\right\}$ is the system of sets (1.6) approximating the reachable set $X_{\alpha}\left(t^{*}, t_{*}, X_{*}\right), \alpha \in \mathscr{L}$, of the differential inclusion (1.4). Then, under the conditions $\boldsymbol{A}$ and $\boldsymbol{B}$ on system (1.1), the following estimates hold:

$$
\begin{gathered}
d\left(\widetilde{X}_{\alpha}^{\Gamma_{*}}\left(\tau_{i+1}\right), \widetilde{X}_{\beta}^{\Gamma_{*}}\left(\tau_{i+1}\right)\right) \leqslant\left(1+\sum_{s=0}^{i-1} e^{\sum_{k=i-s}^{i} L\left(\tau_{k}\right) \Delta_{k}}\right) \Delta \omega^{*}(\|\alpha-\beta\|), \\
d\left(\widetilde{X}_{\alpha}^{\Gamma_{*}}\left(\tau_{i+1}\right), \widetilde{X}_{\beta}^{\Gamma_{*}}\left(\tau_{i+1}\right)\right) \leqslant e^{\sum_{k=0}^{i} L\left(\tau_{k}\right) \Delta_{k}}\left(\tau_{i+1}-\tau_{0}\right) \omega^{*}(\|\alpha-\beta\|),
\end{gathered}
$$




$$
\begin{aligned}
& d\left(\widetilde{X}_{\alpha}^{\Gamma_{*}}\left(t^{*}\right), \widetilde{X}_{\beta}^{\Gamma_{*}}\left(t^{*}\right)\right) \leqslant e^{\sum_{k=0}^{N-1} L\left(\tau_{k}\right) \Delta_{k}}\left(t^{*}-t_{*}\right) \omega^{*}(\|\alpha-\beta\|), \\
& d\left(\widetilde{X}_{\alpha}^{\Gamma_{*}}\left(\tau_{i+1}\right), \widetilde{X}_{\beta}^{\Gamma_{*}^{*}}\left(\tau_{i+1}\right)\right) \leqslant \sum_{k=0}^{i} e^{L k \Delta} \Delta \omega^{*}(\|\alpha-\beta\|), \\
& d\left(\widetilde{X}_{\alpha}^{\Gamma_{*}}\left(\tau_{i+1}\right), \widetilde{X}_{\beta}^{\Gamma_{*}}\left(\tau_{i+1}\right)\right) \leqslant e^{L \cdot\left(\tau_{i+1}-\tau_{0}\right)} \cdot\left(\tau_{i+1}-\tau_{0}\right) \omega^{*}(\|\alpha-\beta\|), \\
& d\left(\widetilde{X}_{\alpha}^{\Gamma_{*}}\left(t^{*}\right), \widetilde{X}_{\beta}^{\Gamma_{*}}\left(t^{*}\right)\right) \leqslant e^{L\left(t^{*}-t_{*}\right)}\left(t^{*}-t_{*}\right) \omega^{*}(\|\alpha-\beta\|) .
\end{aligned}
$$

From estimate (1.28), we derive estimates (1.29) and (1.30).

Let us write one more important estimate that follows from (1.28):

$$
d\left(\tilde{X}_{\alpha}^{\Gamma_{*}}\left(\tau_{i+1}, \tilde{X}_{\beta}^{\Gamma_{*}}\left(\tau_{i+1}\right)\right) \leqslant \frac{e^{(i+1) L \Delta}-1}{e^{L \Delta}-1} \Delta \omega^{*}(\|\alpha-\beta\|), \quad i=\overline{0, N-1} .\right.
$$

Let us estimate from above the right-hand side of this inequality, assuming that, along with the conditions $\mathbf{A}$ and $\mathbf{B}$ on system (1.1), the following condition on the partition $\Gamma_{*}$ of the time interval $\left[t_{*}, t^{*}\right]$ holds.

C. The diameter of the partition $\Gamma_{*}$ satisfies the relation

$$
0<\Delta=\Delta\left(\Gamma_{*}\right)<L^{-1} \ln \left(1+\frac{3}{2} L \Delta\right) .
$$

Under the condition $\mathbf{C}$, the following inequalities are valid:

$$
\begin{gathered}
\frac{e^{(i+1) L \Delta}-1}{e^{L \Delta}-1}<\frac{e^{L \Delta} \cdot e^{L\left(\tau_{i+1}-\tau_{0}\right)}-1}{L \Delta} \\
<\frac{(1+3 / 2 \cdot L \Delta) e^{L\left(\tau_{i+1}-\tau_{0}\right)}-1}{L \Delta}=\frac{e^{L\left(\tau_{i+1}-\tau_{0}\right)}-1}{L \Delta}+\frac{3}{2} e^{L\left(\tau_{i+1}-\tau_{0}\right)} .
\end{gathered}
$$

Taking this inequality into account, we obtain

$$
\begin{gathered}
d\left(\widetilde{X}_{\alpha}^{\Gamma_{*}^{*}}\left(\tau_{i+1}\right), \widetilde{X}_{\beta}^{\Gamma_{*}}\left(\tau_{i+1}\right)\right)<L^{-1}\left(e^{L\left(\tau_{i+1}-\tau_{0}\right)}-1\right) \omega^{*}(\|\alpha-\beta\|)+ \\
+\frac{3}{2} e^{L\left(\tau_{i+1}-\tau_{0}\right)} \Delta \omega^{*}(\|\alpha-\beta\|), \quad i=\overline{0, N-1}
\end{gathered}
$$

As a result, the following statement is true.

Theorem 1. Let $\left[t_{*}, t^{*}\right] \subset\left[t_{0}, \vartheta\right]$ and $X_{*} \in \operatorname{comp}\left(\mathbb{R}^{n}\right)$. Then, under the conditions $\boldsymbol{A}, \boldsymbol{B}$, and $\boldsymbol{C}$ on system (1.1), the sets $X_{\alpha}\left(t^{*}\right)=X_{\alpha}\left(t^{*}, t_{*}, X_{*}\right)$ and $X_{\beta}\left(t^{*}\right)=X_{\beta}\left(t^{*}, t_{*}, X_{*}\right)$, $\alpha$ and $\beta$ from $\mathscr{L}$, satisfy estimate (1.31).

Obviously, for small $\Delta=\Delta\left(\Gamma_{*}\right)$, the strict estimate (1.31) will turn into the following estimate:

$$
d\left(\widetilde{X}_{\alpha}^{\Gamma_{*}}\left(\tau_{i+1}\right), \widetilde{X}_{\beta}^{\Gamma_{*}}\left(\tau_{i+1}\right)\right) \leqslant L^{-1}\left(e^{L\left(\tau_{i+1}-\tau_{0}\right)}-1\right) \omega^{*}(\|\alpha-\beta\|), \quad i=\overline{0, N-1} .
$$

In particular, the following statement is true.

Assertion 1. Assume that $\left[t_{*}, t^{*}\right] \subset\left[t_{0}, \vartheta\right]$ and $X_{*} \in \operatorname{comp}\left(\mathbb{R}^{n}\right)$. Then, under the conditions $\boldsymbol{A}, \boldsymbol{B}$, and $\boldsymbol{C}$ on system (1.1), the sets $X_{\alpha}\left(t^{*}\right)=X_{\alpha}\left(t^{*}, t_{*}, X_{*}\right)$ and $X_{\beta}\left(t^{*}\right)=X_{\beta}\left(t^{*}, t_{*}, X_{*}\right)$, $\alpha$ and $\beta$ from $\mathscr{L}$, satisfy the estimate

$$
d\left(\widetilde{X}_{\alpha}^{\Gamma_{*}}\left(t^{*}\right), \widetilde{X}_{\beta}^{\Gamma_{*}}\left(t^{*}\right)\right) \leqslant L^{-1}\left(e^{L\left(t^{*}-t_{*}\right)}-1\right) \omega^{*}(\|\alpha-\beta\|) .
$$


The question arises, at what ratios between the numbers $L$ and $\left(t^{*}-t_{*}\right)$ one or another of estimates (1.30) and (1.32) is better. To answer it, let us compare the numbers $L^{-1}\left(e^{L\left(t^{*}-t_{*}\right)}-1\right)$ and $e^{L\left(t^{*}-t_{*}\right)}\left(t^{*}-t_{*}\right)$, i.e., compare $e^{L\left(t^{*}-t_{*}\right)}-1$ and $e^{L\left(t^{*}-t_{*}\right)} L\left(t^{*}-t_{*}\right)$.

Assuming that $L\left(t^{*}-t_{*}\right)=\rho>0$, we come to the comparison of $e^{\rho} \cdot(1-\rho)$ and 1 for $\rho \geqslant 0$.

Since the function $e^{\rho} \cdot(1-\rho)$ equals 1 for $\rho=0$ and decreases on $[0, \infty)$, we get

$$
e^{\rho} \cdot(1-\rho)<1, \quad \rho>0
$$

and therefore

$$
L^{-1}\left(e^{L\left(t^{*}-t_{*}\right)}-1\right)<e^{L\left(t^{*}-t_{*}\right)}\left(t^{*}-t_{*}\right)
$$

for every $L \in(0, \infty)$ and $\left(t^{*}-t_{*}\right)>0$.

This means that estimate (1.32) is more precise than estimate (1.30) for sufficiently small $\Delta=\Delta\left(\Gamma_{*}\right)$.

We have considered the case $X_{*}=\left\{x_{*}\right\},\left(t_{*}, x_{*}\right) \in D$, and received estimates (1.25)-(1.30). Estimates (1.25)-(1.30) are also true in the general case $X_{*} \in \operatorname{comp}\left(\mathbb{R}^{n}\right),\left(t_{*}, X_{*}\right) \subset D$.

Bearing in mind the general case, we choose from (1.25)-(1.30) estimate (1.27) for the following reasons. Along with the sets $\widetilde{X}_{\alpha}^{\Gamma_{*}}\left(t^{*}\right)$ and $\widetilde{X}_{\beta}^{\Gamma_{*}}\left(t^{*}\right)$ included in $(1.27)$, consider the reachable sets $X_{\alpha}\left(t^{*}\right)=X_{\alpha}\left(t^{*}, t_{*}, X_{*}\right)$ and $X_{\beta}\left(t^{*}\right)=X_{\beta}\left(t^{*}, t_{*}, X_{*}\right)$ of the differential inclusion (1.4).

We are looking for upper bounds for the values $d\left(X_{\alpha}\left(t^{*}\right), \widetilde{X}_{\alpha}^{\Gamma_{*}}\left(t^{*}\right)\right)$ and $d\left(X_{\beta}\left(t^{*}\right), \tilde{X}_{\beta}^{\Gamma_{*}}\left(t^{*}\right)\right)$, where $\alpha$ and $\beta$ are from $\mathscr{L}$. It is known that, under the conditions $\mathbf{A}$ and $\mathbf{B}$ on system (1.1), these estimates are of the form

$$
\begin{aligned}
& d\left(X_{\alpha}\left(t^{*}\right), \widetilde{X}_{\alpha}^{\Gamma_{*}}\left(t^{*}\right)\right) \leqslant e^{L \cdot\left(t^{*}-t_{*}\right)}\left(t^{*}-t_{*}\right)\left(\omega^{*}(\Delta)+L K \Delta\right), \\
& d\left(X_{\beta}\left(t^{*}\right), \widetilde{X}_{\beta}^{\Gamma_{*}^{*}}\left(t^{*}\right)\right) \leqslant e^{L \cdot\left(t^{*}-t_{*}\right)}\left(t^{*}-t_{*}\right)\left(\omega^{*}(\Delta)+L K \Delta\right) ;
\end{aligned}
$$

here $L \in(0, \infty)$ is defined on p. $127, K=\max _{(t, x, u, \alpha) \in D \times P \times \mathscr{L}}\left\|f_{\alpha}(t, x, u)\right\| \in(0, \infty)$, and $\Delta=\Delta\left(\Gamma_{*}\right)$.

Remark 2. It can be shown that, along with estimates (1.33), there are more subtle estimates:

$$
\begin{aligned}
& d\left(X_{\alpha}\left(t^{*}\right), \widetilde{X}_{\alpha}^{\Gamma_{*}}\left(t^{*}\right)\right) \leqslant e^{\int_{t_{*}^{*}}^{t^{*}} L(t) d t}\left(t^{*}-t_{*}\right)\left(\omega^{*}(\Delta)+L K \Delta\right), \\
& d\left(X_{\beta}\left(t^{*}\right), \widetilde{X}_{\beta}^{\Gamma_{*}}\left(t^{*}\right)\right) \leqslant e^{\int_{t_{*}^{*}}^{t^{*}} L(t) d t}\left(t^{*}-t_{*}\right)\left(\omega^{*}(\Delta)+L K \Delta\right) .
\end{aligned}
$$

Taking into account (1.27) and (1.33), we get

$$
\begin{gathered}
d\left(X_{\alpha}\left(t^{*}\right), X_{\beta}\left(t^{*}\right)\right) \leqslant d\left(X_{\alpha}\left(t^{*}\right), \tilde{X}_{\alpha}^{\Gamma_{*}}\left(t^{*}\right)\right)+d\left(\tilde{X}_{\alpha}^{\Gamma_{*}}\left(t^{*}\right), \tilde{X}_{\beta}^{\Gamma_{*}^{*}}\left(t^{*}\right)\right)+d\left(\widetilde{X}_{\beta}^{\Gamma_{*}}\left(t^{*}\right), X_{\beta}\left(t^{*}\right)\right) \\
\leqslant e^{\sum_{k=1}^{N-1} L\left(\tau_{k}\right) \Delta_{k}} \cdot\left(t^{*}-t_{*}\right) \omega^{*}(\|\alpha-\beta\|)+2 e^{L \cdot\left(t^{*}-t_{*}\right)} \cdot\left(\omega^{*}(\Delta)+L K \Delta\right),
\end{gathered}
$$

where $\alpha$ and $\beta$ from $\mathscr{L}$.

Since this estimate holds for any partitions $\Gamma_{*}$ of the interval $\left[t_{*}, t^{*}\right]$, letting the diameter $\Delta=\Delta\left(\Gamma_{*}\right)$ of the partition $\Gamma_{*}$ tend to zero, we obtain

$$
d\left(X_{\alpha}\left(t^{*}\right), X_{\beta}\left(t^{*}\right)\right) \leqslant e^{\int_{t_{*}^{*}}^{t^{*}} L(t) d t} \cdot\left(t^{*}-t_{*}\right) \cdot \omega^{*}(\|\alpha-\beta\|)
$$

here $\int_{t_{*}}^{t^{*}} L(t) d t$ is the Riemann integral of the function $L(t)$ over the interval $\left[t_{*}, t^{*}\right] \subset\left[t_{0}, \vartheta\right]$.

Now let us turn to the interval $\left[t_{0}, \vartheta\right]$, on which the control system (1.1) and the differential inclusion (1.4) are initially considered. 
Assume that in the previous calculations $t_{*}=t_{0}, t^{*}=t \in\left[t_{0}, \vartheta\right], X_{*}=X^{(0)} \in \operatorname{comp}\left(\mathbb{R}^{n}\right)$, and $\left(t_{0}, X^{(0)}\right) \subset D$, where $X^{(0)}$ is the initial set for system (1.1) and the differential inclusion (1.4), so that the reachable sets $X_{\alpha}(t)$ and $X_{\beta}(t)$ of the differential inclusion (1.4) become $X_{\alpha}(t)=X_{\alpha}\left(t, t_{0}, X^{(0)}\right)$ and $X_{\beta}(t)=X_{\beta}\left(t, t_{0}, X^{(0)}\right)$.

For these sets, we write estimate (1.34):

$$
d\left(X_{\alpha}(t), X_{\beta}(t)\right) \leqslant e^{\int_{t_{0}}^{t} L(\tau) d \tau} \cdot\left(t-t_{0}\right) \omega^{*}(\|\alpha-\beta\|),
$$

where $t \in\left[t_{0}, \vartheta\right]$ and $\alpha, \beta \in \mathscr{L}$.

We also introduce the partition $\Gamma=\left\{t_{0}, t_{1}, \ldots, t_{i}, \ldots, t_{N}=\vartheta\right\}$ of the interval $\left[t_{0}, \vartheta\right]$ with the diameter $\Delta=\Delta(\Gamma)=t_{i+1}-t_{i}=N^{-1}\left(\vartheta-t_{0}\right)$.

Along with the reachable sets $X_{\alpha}(t), \alpha \in \mathscr{L}, t \in\left[t_{0}, \vartheta\right]$, we consider the integral funnel

$$
X_{\alpha}\left(t_{0}, X^{(0)}\right)=\bigcup_{t \in\left[t_{0}, \vartheta\right]}\left(t, X_{\alpha}(t)\right), \quad \alpha \in \mathscr{L},
$$

of the differential inclusion (1.4).

Assume that

$$
X_{\alpha}^{\Gamma}\left(t_{0}, X^{(0)}\right)=\bigcup_{t_{i} \in \Gamma}\left(t_{i}, X_{\alpha}\left(t_{i}\right)\right), \tilde{X}_{\alpha}^{\Gamma}\left(t_{0}, X^{(0)}\right)=\bigcup_{t_{i} \in \Gamma}\left(t_{i}, \tilde{X}_{\alpha}^{\Gamma}\left(t_{i}\right)\right)
$$

are sets in $D$, where $\widetilde{X}_{\alpha}^{\Gamma}\left(t_{i}\right)$ are defined on p. 122 by the recurrent relations with $\tau_{0}=t_{0}$ and $\tilde{X}_{\alpha}^{\Gamma}\left(t_{0}\right)=\tilde{X}_{\alpha}^{\Gamma}\left(\tau_{0}\right)=X^{(0)}$.

Here the sets $X_{\alpha}^{\Gamma}\left(t_{0}, X^{(0)}\right)$ and $\widetilde{X}_{\alpha}^{\Gamma}\left(t_{0}, X^{(0)}\right)$ are some approximations of the integral funnel $X_{\alpha}\left(t_{0}, X^{(0)}\right), \alpha \in \mathscr{L}$, discrete by the parameter $t \in\left[t_{0}, \vartheta\right]$.

From the estimate

$$
d\left(X_{\alpha}\left(t_{i}\right), \widetilde{X}_{\alpha}^{\Gamma}\left(t_{i}\right)\right) \leqslant e^{L \cdot\left(t_{i}-t_{0}\right)}\left(t_{i}-t_{0}\right)\left(\omega^{*}(\Delta)+L K \Delta\right), \quad i=\overline{1, N}, \quad \alpha \in \mathscr{L},
$$

we obtain the estimate

$$
d\left(X_{\alpha}^{\Gamma}\left(t_{0}, X^{(0)}\right), \widetilde{X}_{\alpha}^{\Gamma}\left(t_{0}, X^{(0)}\right)\right) \leqslant e^{L \cdot\left(\vartheta-t_{0}\right)}\left(\vartheta-t_{0}\right)\left(\omega^{*}(\Delta)+L K \Delta\right) ;
$$

here $L$ is defined on p. 127 and $K$ on p. 129.

Since the following inequality holds for each interval $\left[t_{i}, t_{i+1}\right]$ of the partition $\Gamma$, every $t \in$ $\left[t_{i}, t_{i+1}\right]$, and every $\alpha \in \mathscr{L}:$

$$
d\left(\left(t, X_{\alpha}(t)\right),\left(t_{i}, X_{\alpha}\left(t_{i}\right)\right)\right) \leqslant(1+K) \Delta,
$$

we have

$$
d\left(X_{\alpha}\left(t_{0}, X^{(0)}\right), X_{\alpha}^{\Gamma}\left(t_{0}, X^{(0)}\right)\right) \leqslant(1+K) \Delta .
$$

Considering estimates (1.36) and (1.37), we get

$$
d\left(X_{\alpha}\left(t_{0}, X^{(0)}\right), \widetilde{X}_{\alpha}^{\Gamma}\left(t_{0}, X^{(0)}\right)\right) \leqslant e^{L \cdot\left(\vartheta-t_{0}\right)}\left(\vartheta-t_{0}\right)\left(\omega^{*}(\Delta)+L K \Delta\right)+(1+K) \Delta .
$$

Obviously, using the technique of obtaining estimates described above, we can replace estimate (1.38) with a more accurate one:

$$
\begin{gathered}
d\left(X_{\alpha}\left(t_{0}, X^{(0)}\right), \widetilde{X}_{\alpha}^{\Gamma}\left(t_{0}, X^{(0)}\right)\right) \leqslant e^{\sum_{i=0}^{N-1} L\left(t_{i}\right) \Delta_{i}} \cdot\left(\vartheta-t_{0}\right)\left(\omega^{*}(\Delta)+L K \Delta\right)+(1+K) \Delta, \\
\Delta_{i}=\Delta=\Delta(\Gamma), \quad i \in \overline{0, N-1}, \quad \alpha \in \mathscr{L} .
\end{gathered}
$$

Inequality (1.35) implies the following statement for the integral funnels $X_{\alpha}\left(t_{0}, X^{(0)}\right)$ and $X_{\beta}\left(t_{0}, X^{(0)}\right)$.

Theorem 2. Let the control system (1.1) satisfy the conditions $\boldsymbol{A}, \boldsymbol{B}$, and $\boldsymbol{C}$, and let $X^{(0)} \in \operatorname{comp}\left(\mathbb{R}^{n}\right)$. Then the integral funnels $X_{\alpha}\left(t_{0}, X^{(0)}\right)$ and $X_{\beta}\left(t_{0}, X^{(0)}\right)$ satisfy the inequality

$$
d\left(X_{\alpha}\left(t_{0}, X^{(0)}\right), X_{\beta}\left(t_{0}, X^{(0)}\right)\right) \leqslant e^{\int_{t_{0}}^{\vartheta} L(t) d t} \cdot\left(\vartheta-t_{0}\right) \omega^{*}(\|\alpha-\beta\|), \quad \alpha, \beta \in \mathscr{L} .
$$




\section{Problems of targeting integral funnels to target sets in $\mathbb{R}^{2}$}

In this section, we restrict ourselves to considering system (1.1) and the differential inclusion (1.4) in the space $\mathbb{R}^{2}$. Let us study problems of targeting integral funnels $X_{\alpha}\left(t_{0}, x_{0}\right), \alpha \in \mathscr{L}, x_{0} \in$ $X^{(0)}$, and their approximations $\widetilde{X}_{\alpha}^{\Gamma}\left(t_{0}, x_{0}\right)$ to target sets in $\mathbb{R}^{2}$. We formulate some of these problems using the concept of the area of a set in $\mathbb{R}^{2}$. In this regard, we will study questions concerning the approximate calculation of the areas of reachable sets $X_{\alpha}\left(t, t_{0}, x_{0}\right), x_{0} \in X^{(0)} \in \operatorname{comp}\left(\mathbb{R}^{2}\right)$, and sets associated with $X_{\alpha}\left(t, t_{0}, x_{0}\right)$. In this case, we use the estimates of the Hausdorff distances obtained in Section 1.

Let us start the study of targeting problems by considering the individual integral funnels $X_{\alpha}\left(t_{0}, X^{(0)}\right), X^{(0)} \in \operatorname{comp}\left(\mathbb{R}^{2}\right)$. Of course, the funnels $X_{\alpha}\left(t_{0}, x_{0}\right), \alpha \in \mathscr{L}, x_{0} \in X^{(0)}$, also belong to the class of these funnels. Thus, the estimates of the Hausdorff distances obtained for integral funnels $X_{\alpha}\left(t_{0}, X^{(0)}\right), \alpha \in \mathscr{L}$, also hold for funnels $X_{\alpha}\left(t_{0}, x_{0}\right), \alpha \in \mathscr{L}$.

Let us take an arbitrary funnel $X_{\alpha}\left(t_{0}, X^{(0)}\right), \alpha \in \mathscr{L}, X^{(0)} \in \operatorname{comp}\left(\mathbb{R}^{2}\right)$, and its approximating set $\widetilde{X}_{\alpha}^{\Gamma}\left(t_{0}, X^{(0)}\right)=\bigcup_{t_{i} \in \Gamma}\left(t_{i}, \widetilde{X}_{\alpha}^{\Gamma}\left(t_{i}\right)\right)$ in $D$ corresponding to the partition $\Gamma=\left\{t_{0}, t_{1}, \ldots, t_{i}, \ldots, t_{N}=\vartheta\right\}$ $\left(t_{i+1}-t_{i}=\Delta_{i}=\Delta=\Delta(\Gamma), i=\overline{0, N-1}\right)$.

The mismatch between the time sections $X_{\alpha}\left(t_{i}\right)$ and $\widetilde{X}_{\alpha}^{\Gamma}\left(t_{i}\right), t_{i} \in \Gamma$, of the sets $X_{\alpha}\left(t_{0}, X^{(0)}\right)$ and $\widetilde{X}_{\alpha}^{\Gamma}\left(t_{0}, X^{(0)}\right)$ is restricted by the estimate

$$
d\left(X_{\alpha}\left(t_{i}\right), \widetilde{X}_{\alpha}^{\Gamma}\left(t_{i}\right)\right) \leqslant e^{\sum_{j=0}^{N-1} L\left(t_{j}\right) \Delta_{j}} \cdot\left(K \Delta \sum_{j=0}^{N-1} L\left(t_{j}\right) \Delta_{j}+\left(t_{j}-t_{0}\right) \omega^{*}(\Delta)\right) .
$$

Along with the set $\widetilde{X}_{\alpha}^{\Gamma}\left(t_{0}, X^{(0)}\right)$ and its sections $\widetilde{X}_{\alpha}^{\Gamma}\left(t_{i}\right), t_{i} \in \Gamma$, we consider the set $\widetilde{X}_{\beta}^{\Gamma}\left(t_{0}, X^{(0)}\right)$, $\beta \in \mathscr{L}$, and its sections $\widetilde{X}_{\beta}^{\Gamma}\left(t_{i}\right), t_{i} \in \Gamma$. The following estimate is valid:

$$
d\left(\widetilde{X}_{\alpha}^{\Gamma}\left(t_{i}\right), \widetilde{X}_{\beta}^{\Gamma}\left(t_{i}\right)\right) \leqslant e^{\sum_{j=0}^{N-1} L\left(t_{j}\right) \Delta_{j}} \cdot\left(t_{i}-t_{0}\right) \omega^{*}(\|\alpha-\beta\|) .
$$

Estimates (2.1) and (2.2) implpy

$$
d\left(X_{\alpha}\left(t_{i}\right), \tilde{X}_{\beta}^{\Gamma}\left(t_{i}\right)\right) \leqslant \varkappa(\Delta,\|\alpha-\beta\|),
$$

where

$$
\begin{gathered}
\varkappa(\Delta, \rho)=e^{\sum_{j=0}^{N-1} L\left(t_{j}\right) \Delta_{j}}\left(\left(\vartheta-t_{0}\right) \omega^{*}(\rho)+\left(\vartheta-t_{0}\right) \omega^{*}(\Delta)+K \Delta \sum_{j=0}^{N-1} L\left(t_{j}\right) \Delta_{j}\right), \\
\alpha, \beta \in \mathscr{L}, \quad t_{i} \in \Gamma, \quad \rho \in(0, \infty) .
\end{gathered}
$$

We will use estimates (2.1)-(2.3) for studying problems of targeting integral funnels to target sets. These estimates will also be taken into account when estimating the mismatch of sets of the type of reachable sets in $\mathbb{R}^{2}$.

Let us formulate these targeting problems.

Assume that a finite set $\mathcal{T}$ of times $\eta_{1}, \eta_{2}, \ldots, \eta_{N_{*}}$ from the interval $\left[t_{0}, \vartheta\right]$ is given and the partition $\Gamma=\left\{t_{0}, t_{1}, \ldots, t_{i}, \ldots, t_{N}=\vartheta\right\}$ considered previously contains this set $\mathcal{T}$.

Assume that compact sets $X^{(0)}, X^{(\vartheta)}$, and $\Phi^{(k)}$ in $\mathbb{R}^{2}$ are given, where each set $\Phi^{(k)}$ corresponds to its time $\eta_{k} \in \mathcal{T}$; moreover, the sets $X^{(0)}, X^{(\vartheta)}$, and $\Phi^{(k)}, \eta_{k} \in \mathcal{T}$, have rectifiable boundaries $\partial X^{(0)}, \partial X^{(\vartheta)}$, and $\partial \Phi^{(k)}, \eta_{k} \in \mathcal{T}$.

Here we assume that $\Phi^{(k)}=\Phi\left(\eta_{k}\right), \eta_{k} \in \mathcal{T}$, where the set $\Phi(t) \in \operatorname{comp}\left(\mathbb{R}^{2}\right), t \in\left[t_{0}, \vartheta\right]$, is interpreted by us as an obstacle to system (1.1). 
Problem 1 on targeting integral funnels (strict setting). It is required to find a pair $\left(\alpha_{*}, x_{*}\right) \in \mathscr{L} \times X^{(0)}$ such that the following relations hold:

$$
X^{(\vartheta)} \subset X_{\alpha_{*}}\left(\vartheta, t_{0}, x_{*}\right), \quad \Phi^{(k)} \cap X_{\alpha_{*}}\left(\eta_{k}, t_{0}, x_{*}\right)=\varnothing, \quad \eta_{k} \in \mathcal{T} .
$$

Exact computation of the sets $X_{\alpha}\left(t_{i}, t_{0}, x_{0}\right), \alpha \in \mathscr{L}, t_{i} \in \Gamma, x_{0} \in X^{(0)}$ is not possible due to the complexity of the system dynamics (1.1). In particular, it is impossible to compute the sets $X_{\alpha_{*}}\left(\vartheta, t_{0}, x_{*}\right)$ and $X_{\alpha_{*}}\left(\eta_{k}, t_{0}, x_{*}\right), \eta_{k} \in \mathcal{T}$. Also in the case when, for example, one of the sets $\mathscr{L}$ and $X^{(0)}$ is infinite, the complete enumeration of all pairs $(\alpha, x) \in \mathscr{L} \times X^{(0)}$ is impossible.

Therefore, it makes sense to go from the statement of Problem 1 to a statement in terms of the sets $\widetilde{X}_{\alpha}^{\Gamma}\left(t_{i}, t_{0}, x_{0}\right), \alpha \in \mathscr{L}, t_{i} \in \Gamma, x_{0} \in X^{(0)}$. Moreover, under the sets $\widetilde{X}_{\alpha}^{\Gamma}\left(t_{i}, t_{0}, x_{0}\right)$ we understand time sections of the sets $\widetilde{X}_{\alpha}^{\Gamma}\left(t_{0}, x_{0}\right), \alpha \in \mathscr{L}, x_{0} \in X^{(0)}$, corresponding to the times $t_{i} \in \Gamma$.

More precisely, we assume that there are given $\varepsilon, \rho$, and $\sigma$ from $(0, \infty)$ and finite sets corresponding to the numbers $\rho$ and $\sigma$ in the sets $\mathscr{L}$ and $X^{(0)}$, a $\rho$-net $\mathscr{L}^{(\rho)}=\left\{\alpha^{(r)}: r=\overline{1, r_{*}}\right\}$ and a $\sigma$-net $X^{(\sigma)}=\left\{x^{(s)}: s=\overline{1, s_{*}}\right\}$.

Problem $1^{(\varepsilon)}$ on targeting integral funnels. It is required to find a pair $\left(\alpha^{(r)}, x^{(s)}\right) \in$ $\mathscr{L}^{(\rho)} \times X^{(\sigma)}$ such that the following relations hold:

$$
X^{(\vartheta)} \subset \widetilde{X}_{\alpha^{(r)}}^{\Gamma}\left(\vartheta, t_{0}, x^{(s)}\right)_{\varepsilon}, \quad \Phi_{\varepsilon}^{(k)} \cap \widetilde{X}_{\alpha^{(r)}}^{\Gamma}\left(\eta_{k}, t_{0}, x^{(s)}\right)=\varnothing, \quad \eta_{k} \in \mathcal{T} .
$$

For Problems 1 or $1^{(\varepsilon)}$ formulated for a particular system (1.1), it may turn out that there is no solution. Taking into account such situations, we formulate the targeting problem in a less strict setting, using the concept of the area of a set in $\mathbb{R}^{2}$. At the same time, we assume that such a formulation does not contradict the meaning of the original real targeting problem.

First, we give a statement in terms of ideal reachable sets $X_{\alpha}\left(t_{i}, t_{0}, x_{0}\right), \alpha \in \mathscr{L}, x_{0} \in X^{(0)}$, $t_{i} \in \Gamma$.

Let us introduce the notation

$$
\begin{aligned}
J^{(1)}(\alpha, x) & =\sum_{\eta_{k} \in \mathcal{T}} s\left(\Phi^{(k)} \backslash X_{\alpha}\left(\eta_{k}, t_{0}, x\right)\right), \\
J^{(2)}(\alpha, x) & =s\left(X^{(\vartheta)} \cap X_{\alpha}\left(\vartheta, t_{0}, x\right)\right), \\
\alpha & \in \mathscr{L}, \quad x \in X^{(0)}
\end{aligned}
$$

here $s(Y)$ is the area of the set $Y \in \operatorname{comp}\left(\mathbb{R}^{2}\right)$, by which we mean the Lebesgue measure (see, e.g., [4, Ch. 2, Sect. 2.5]) of the compact set $Y$ in $\mathbb{R}^{2}$.

Let us fix $\lambda_{1}$ and $\lambda_{2}$ from $[0,1], \lambda_{1}+\lambda_{2}=1$.

Let us clarify once again that, under a strict setting of the problem of targeting integral funnels of the differential inclusion (1.4), we mean a setting in which an integral funnel $X_{\alpha}\left(t_{0}, x^{(0)}\right), \alpha \in \mathscr{L}$, should not intersect an obstacle $\Phi(t), t \in\left[t_{0}, \vartheta\right]$; in the worst case, it can only touch its boundary $\partial \Phi(t), t \in\left[t_{0}, \vartheta\right]$. In this case, the integral funnel $X_{\alpha}\left(t_{0}, x^{(0)}\right), \alpha \in \mathscr{L}$, must completely cover the target set $X^{(\vartheta)}$ at the terminal time $\vartheta$.

The soft setting of the targeting problem allows the integral funnel $X_{\alpha}\left(t_{0}, x^{(0)}\right)$ to creep on the obstacle $\Phi(t), t \in\left[t_{0}, \vartheta\right]$ and admits incomplete coverage of the target set $X^{(\vartheta)}$ by the integral funnel $X_{\alpha}\left(t_{0}, x^{(0)}\right)$ (more precisely, by its latter section $X_{\alpha}\left(\vartheta, t_{0}, x^{(0)}\right)$ ) at the time $\vartheta$. However, this involves some quantitative estimates of the effectiveness of the integral funnel $X_{\alpha}\left(t_{0}, x^{(0)}\right)$ when solving the problem of targeting $X^{(\vartheta)}$. These quantitative estimates are associated with calculating the areas of sets in the space $\mathbb{R}^{2}$.

We assume that $J(\alpha, x)=\lambda_{1} J^{(1)}(\alpha, x)+\lambda_{2} J^{(2)}(\alpha, x)$. 
Problem 2 on targeting integral funnels (soft setting). It is required to find a pair $\left(\alpha^{*}, x^{*}\right) \in \mathscr{L} \times X^{(0)}$ such that the following relation is true:

$$
J\left(\alpha^{*}, x^{*}\right)=\max _{(\alpha, x) \in \mathscr{L} \times X^{(0)}} J(\alpha, x) .
$$

Since we are not able to solve Problem 2 exactly for the same reasons as Problem 1, we formulate and solve some approximation problem in which, instead of the sets $\mathscr{L}$ and $X^{(0)}$, in the cases where they are not finite, there are their finite nets $\mathscr{L}^{(\rho)}$ and $X^{(\sigma)}$ and, instead of (ideal) reachable sets $X_{\alpha}\left(t, t_{0}, x_{0}\right), \alpha \in \mathscr{L}, x_{0} \in X^{(0)}$, there are their approximations $\widetilde{X}_{\alpha^{(r)}}^{\Gamma}\left(t_{i}, t_{0}, x^{(s)}\right),\left(\alpha^{(r)}, x^{(s)}\right) \in$ $\mathscr{L}^{(\rho)} \times X^{(\sigma)}$

Let us introduce the notation

$$
\begin{gathered}
\widetilde{J}_{\Gamma}^{(1)}(\beta, y)=\sum_{\eta_{k} \in \mathcal{T}} s\left(\Phi^{(k)} \backslash \widetilde{X}_{\beta}^{\Gamma}\left(\eta_{k}, t_{0}, y\right)\right), \\
\widetilde{J}_{\Gamma}^{(2)}(\beta, y)=s\left(X^{(\vartheta)} \cap \widetilde{X}_{\beta}^{\Gamma}\left(\vartheta, t_{0}, y\right)\right), \\
(\beta, y) \in \mathscr{L}^{(\rho)} \times X^{(\sigma)} .
\end{gathered}
$$

We assume that $\widetilde{J}_{\Gamma}(\beta, y)=\lambda_{1} \widetilde{J}_{\Gamma}^{(1)}(\beta, y)+\lambda_{2} \widetilde{J}_{\Gamma}^{(2)}(\beta, y)$.

Problem 3 on targeting integral funnels (soft setting). It is required to find a pair $\left(\beta^{*}, y^{*}\right) \in \mathscr{L}^{(\rho)} \times X^{(\sigma)}$ such that the following relation holds:

$$
\widetilde{J}_{\Gamma}\left(\beta^{*}, y^{*}\right)=\max _{(\beta, y) \in \mathscr{L}^{(\rho)} \times X^{(\sigma)}} \widetilde{J}_{\Gamma}(\beta, y) .
$$

Let us show that, for small $\rho$ and $\sigma$ from $(0, \infty)$, the solution of the approximation Problem 3 is close to the solution of Problem 2. This circumstance justifies replacing Problem 2 with Problem 3. In this case, we understand the proximity of solutions as the proximity of optimal values (2.4) and (2.5) in Problems 2 and 3 and the proximity of optimal pairs in $\mathscr{L} \times X^{(0)}$ and $\mathscr{L}^{(\rho)} \times X^{(\sigma)}$.

So, consider first pairs $(\alpha, x)$ and $(\beta, y)$, where $(\alpha, x)$ is chosen in $\mathscr{L} \times X^{(0)}$ arbitrarily and the pair $(\beta, y) \in \mathscr{L}^{(\rho)} \times X^{(\sigma)}$ is such that $\|\alpha-\beta\| \leqslant \rho$ and $\|x-y\| \leqslant \sigma$.

Let us find an upper bound for the Hausdorff distance

$$
d\left(X_{\alpha}\left(\eta_{k}, t_{0}, x\right), \widetilde{X}_{\beta}^{\Gamma}\left(\eta_{k}, t_{0}, y\right)\right), \quad \eta_{k} \in \mathcal{T} .
$$

In view of $(2.3)$ and the estimate

$$
d\left(\tilde{X}_{\beta}^{\Gamma}\left(\eta_{k}, t_{0}, x\right), \widetilde{X}_{\beta}^{\Gamma}\left(\eta_{k}, t_{0}, y\right)\right) \leqslant e^{\sum_{j=0}^{N-1} L\left(t_{j}\right) \Delta_{j}} \cdot\|x-y\| \leqslant e^{\sum_{j=0}^{N-1} L\left(t_{j}\right) \Delta_{j}} \cdot \sigma,
$$

we have

$$
\begin{gathered}
d\left(X_{\alpha}\left(\eta_{k}, t_{0}, x\right), \tilde{X}_{\beta}^{\Gamma}\left(\eta_{k}, t_{0}, y\right)\right) \leqslant d\left(X_{\alpha}\left(\eta_{k}, t_{0}, x\right), \widetilde{X}_{\beta}^{\Gamma}\left(\eta_{k}, t_{0}, x\right)\right)+d\left(\widetilde{X}_{\beta}^{\Gamma}\left(\eta_{k}, t_{0}, x\right), \widetilde{X}_{\beta}^{\Gamma}\left(\eta_{k}, t_{0}, y\right)\right) \\
\leqslant \varkappa(\Delta, \rho)+e^{\sum_{j=0}^{N-1} L\left(t_{j}\right) \Delta_{j}} \cdot \sigma, \quad \eta_{k} \in \mathcal{T} .
\end{gathered}
$$

For simplicity, we introduce the notation

$$
\varkappa^{\Delta}(\rho, \sigma)=\varkappa(\Delta, \rho)+e^{\sum_{j=0}^{N-1} L\left(t_{j}\right) \Delta_{j}} \cdot \sigma, \quad \rho, \sigma \in(0, \infty) .
$$

Finally, for pairs $(\alpha, x) \in \mathscr{L} \times X^{(0)}$ and $(\beta, y) \in \mathscr{L}^{(\rho)} \times X^{(\sigma)}$ such that $\|\alpha-\beta\| \leqslant \rho$ and $\|x-y\| \leqslant \sigma$, we have the estimate

$$
d\left(X_{\alpha}\left(\eta_{k}, t_{0}, x\right), \tilde{X}_{\beta}^{\Gamma}\left(\eta_{k}, t_{0}, y\right)\right) \leqslant \varkappa^{\Delta}(\rho, \sigma) .
$$


Let us describe the function $\varkappa^{\Delta}(\rho, \sigma)$ in more detail and estimate it from above. The following representation is valid:

$$
\varkappa^{\Delta}(\rho, \sigma)=e^{\sum_{j=0}^{N-1} L\left(t_{j}\right) \Delta_{j}} \cdot\left(\left(\vartheta-t_{0}\right) \omega^{*}(\rho)+\left(\vartheta-t_{0}\right) \omega^{*}(\Delta)+K \Delta \sum_{j=0}^{N-1} L\left(t_{j}\right) \Delta_{j}+\sigma\right) .
$$

Since, by the condition $\mathbf{A}$, the function $L(t) \in(0, \infty)$ is continuous on $\left[t_{0}, \vartheta\right]$, the following estimate is valid for $L \in\left(\max _{t \in\left[t_{0}, \vartheta\right]} L(t), \infty\right)$ :

$$
\varkappa^{\Delta}(\rho, \sigma) \leqslant e^{L \cdot\left(\vartheta-t_{0}\right)}\left(\left(\vartheta-t_{0}\right) \omega^{*}(\rho)+\left(\vartheta-t_{0}\right) \omega^{*}(\Delta)+L K\left(\vartheta-t_{0}\right) \Delta+\sigma\right) .
$$

This estimate implies the limit equality $\lim _{\Delta \downarrow 0, \rho \downarrow 0, \sigma \downarrow 0} \varkappa^{\Delta}(\rho, \sigma)=0$.

We supplement the conditions $\mathbf{A}, \mathbf{B}$ and $\mathbf{C}$ with the following condition.

D. The lengths of the boundaries $\partial X^{(0)}, \partial X^{(\vartheta)}, \partial \Phi^{(k)}, \partial X_{\alpha}\left(t_{i}, t_{0}, x\right)$, and $\partial \widetilde{X}_{\beta}^{\Gamma}\left(t_{i}, t_{0}, y\right)((\alpha, x) \in$ $\left.\mathscr{L} \times X^{(0)},(\beta, y) \in \mathscr{L}^{(\rho)} \times X^{(\sigma)}, \eta_{k} \in \mathcal{T}, t_{i} \in \Gamma\right)$ are bounded from above by some $l^{*} \in(0, \infty)$.

The condition $\mathbf{D}$ holds for many problems on guiding integral funnels, since the lengths of the boundaries $\partial X^{(0)}, \partial X^{(\vartheta)}$, and $\partial \Phi^{(k)}\left(\eta_{k} \in \mathcal{T}\right)$ are bounded, and the lengths of the boundaries $\partial X_{\alpha}\left(t_{i}, t_{0}, x\right)$ and $\partial \widetilde{X}_{\beta}^{\Gamma}\left(t_{i}, t_{0}, y\right), t_{i} \in \Gamma$, do not increase abruptly with increasing the times $t_{i}$. So, for example, the set $X_{\alpha}\left(t, t_{0}, x\right), \alpha \in \mathscr{L}, x \in X^{(0)}$, continuously depends on $t$ on $\left[t_{0}, \vartheta\right]$ (see Sect. 1 , p. 122) and the set $\partial X_{\alpha}\left(t, t_{0}, x\right)$ also continuously depends on $t$ on $\left[t_{0}, \vartheta\right]$ for many control problems. In these problems, it continuously depends on $t$ and the length of the boundary $\partial X_{\alpha}\left(t, t_{0}, x\right)$.

Let

$$
U_{\alpha}\left(\eta_{k}\right)=\operatorname{cl}\left(X_{\alpha}\left(\eta_{k}, t_{0}, x\right)_{\varkappa}(\rho, \sigma) \backslash X_{\alpha}\left(\eta_{k}, t_{0}, x\right)\right)
$$

be the $\varkappa^{\Delta}(\rho, \sigma)$-layer around the set $X_{\alpha}\left(\eta_{k}, t_{0}, x\right)$, and let

$$
\widetilde{U}_{\beta}^{\Gamma}\left(\eta_{k}\right)=\operatorname{cl}\left(\left(\widetilde{X}_{\beta}^{\Gamma}\left(\eta_{k}, t_{0}, y\right)_{\varkappa^{\Delta}(\rho, \sigma)} \backslash \widetilde{X}_{\beta}^{\Gamma}\left(\eta_{k}, t_{0}, y\right)\right)\right.
$$

be the $\varkappa^{\Delta}(\rho, \sigma)$-layer around the set $\widetilde{X}_{\beta}^{\Gamma}\left(\eta_{k}, t_{0}, y\right)$.

Estimate (2.6) implies

$$
\begin{aligned}
& X_{\alpha}\left(\eta_{k}, t_{0}, x\right) \subset \widetilde{X}_{\beta}^{\Gamma}\left(\eta_{k}, t_{0}, y\right) \cup \widetilde{U}_{\beta}^{\Gamma}\left(\eta_{k}\right), \\
& \widetilde{X}_{\beta}^{\Gamma}\left(\eta_{k}, t_{0}, y\right) \subset X_{\alpha}\left(\eta_{k}, t_{0}, x\right) \cup U_{\alpha}\left(\eta_{k}\right) .
\end{aligned}
$$

From inclusions (2.7), we obtain

$$
\begin{array}{ll}
X_{\alpha}\left(\eta_{k}, t_{0}, x\right) \cap \Phi^{(k)} \subset\left(\widetilde{X}_{\beta}^{\Gamma}\left(\eta_{k}, t_{0}, y\right) \cap \Phi^{(k)}\right) \cup\left(\widetilde{U}_{\beta}^{\Gamma}\left(\eta_{k}\right) \cap \Phi^{(k)}\right), & \eta_{k} \in \mathcal{T}, \\
\widetilde{X}_{\beta}^{\Gamma}\left(\eta_{k}, t_{0}, y\right) \cap \Phi^{(k)} \subset\left(X_{\alpha}\left(\eta_{k}, t_{0}, x\right) \cap \Phi^{(k)}\right) \cup\left(U_{\alpha}\left(\eta_{k}\right) \cap \Phi^{(k)}\right), & \eta_{k} \in \mathcal{T} .
\end{array}
$$

Inclusions (2.8) imply the following inequalities for the areas:

$$
\begin{array}{ll}
s\left(X_{\alpha}\left(\eta_{k}, t_{0}, x\right) \cap \Phi^{(k)}\right) \leqslant s\left(\widetilde{X}_{\beta}^{\Gamma}\left(\eta_{k}, t_{0}, y\right) \cap \Phi^{(k)}\right)+s\left(\widetilde{U}_{\beta}^{\Gamma}\left(\eta_{k}\right)\right), & \eta_{k} \in \mathcal{T}, \\
s\left(\widetilde{X}_{\beta}^{\Gamma}\left(\eta_{k}, t_{0}, y\right) \cap \Phi^{(k)}\right) \leqslant s\left(X_{\alpha}\left(\eta_{k}, t_{0}, x\right) \cap \Phi^{(k)}\right)+s\left(U_{\alpha}\left(\eta_{k}\right)\right), & \eta_{k} \in \mathcal{T} .
\end{array}
$$

From inequalities (2.9), we derive the estimate

$$
\begin{gathered}
\left|s\left(X_{\alpha}\left(\eta_{k}, t_{0}, x\right) \cap \Phi^{(k)}\right)-s\left(\widetilde{X}_{\beta}^{\Gamma}\left(\eta_{k}, t_{0}, y\right) \cap \Phi^{(k)}\right)\right| \leqslant \\
\leqslant \max \left(s\left(U_{\alpha}\left(\eta_{k}\right), s\left(\widetilde{U}_{\beta}^{\Gamma}\left(\eta_{k}\right)\right)\right), \quad \eta_{k} \in \mathcal{T} .\right.
\end{gathered}
$$


Let us make a short note about the layers surrounding compact sets in $\mathbb{R}^{2}$; these layers include the sets $U_{\alpha}\left(\eta_{k}\right)$ and $\widetilde{U}_{\beta}^{\Gamma}\left(\eta_{k}\right), \eta_{k} \in \mathcal{T}$.

It is known (see., e.g., [15]) that if $X \in \operatorname{comp}\left(\mathbb{R}^{2}\right)$ is a convex set, then the area $s\left(U_{\varepsilon}\right)$ of the $\varepsilon$-layer $U_{\varepsilon}=\operatorname{cl}\left(X_{\varepsilon} \backslash X\right)$ surrounding $X$ and the length $l(\partial X)$ of the boundary $\partial X$ of the set $X$ are connected as follows:

$$
s\left(U_{\varepsilon}\right)=l(\partial X) \cdot \varepsilon+\pi \cdot \varepsilon^{2} .
$$

If the set $X \in \operatorname{comp}\left(\mathbb{R}^{2}\right)$ is not convex and connected, then the area $s\left(U_{\varepsilon}\right)$ can satisfy the inequality

$$
s\left(U_{\varepsilon}\right) \leqslant l(\partial X) \cdot \varepsilon+\pi \cdot \varepsilon^{2},
$$

which we will use to estimate the areas $s\left(U_{\alpha}\left(\eta_{k}\right), s\left(\widetilde{U}_{\beta}^{\Gamma}\left(\eta_{k}\right)\right), \tau_{k} \in \mathcal{T}\right.$.

Remark 3. We will give examples of non-convex sets for which equality (2.11) turns into inequality (2.12) and examples of non-convex sets for which equality (2.11) is satisfied. We will also demonstrate that the connectedness condition is necessary.

Example 1. Consider the simplest example of a convex set: $X=\left\{x=\left(x_{1}, x_{2}\right):\|x\| \leqslant R\right\}$, where $R>0$ is the radius of the disk $X$ (Fig. 1).

In this case, equality (2.11) is easily verified by direct computation. Indeed,

$$
s\left(U_{\varepsilon}\right)=\pi(R+\varepsilon)^{2}-\pi R^{2}=2 \pi R \varepsilon+\pi \varepsilon^{2} .
$$

Here the length of the boundary $\partial X$ is equal to $l(\partial X)=2 \pi R$ in full accordance with (2.11).

Example 2. Consider a non-convex set (Fig. 2)

$$
\begin{gathered}
X=\left\{x=\left(x_{1}, x_{2}\right): \max \left\{\left|x_{1}\right|,\left|x_{2}\right|\right\} \leqslant R,\right. \\
\left.\left\|x-A^{*}\right\|>R,\left\|x-B^{*}\right\|>R,\left\|x-C^{*}\right\|>R,\left\|x-D^{*}\right\|>R\right\},
\end{gathered}
$$

where $A^{*}=(-R,-R), B^{*}=(-R, R), C^{*}=(R, R)$, and $D^{*}=(R,-R), R>0$.

We denote by $K=\left\{x=\left(x_{1}, x_{2}\right): \max \left\{\left|x_{1}\right|,\left|x_{2}\right|\right\} \leqslant R\right\}$ the square $A^{*} B^{*} C^{*} D^{*}$. In this case, the $\varepsilon$-layer $U_{\varepsilon}$ consists of four semidisks of radius $\varepsilon$ centered in the middle of the segments $A^{*} B^{*}$, $B^{*} C^{*}, C^{*} D^{*}$, and $A^{*} D^{*}$ and the four curvilinear sets

$$
\begin{aligned}
& U_{1}=\left(B\left(A^{*}, R\right) \backslash B\left(A^{*}, R-\varepsilon\right)\right) \cap K, \\
& U_{2}=\left(B\left(B^{*}, R\right) \backslash B\left(B^{*}, R-\varepsilon\right)\right) \cap K, \\
& U_{3}=\left(B\left(C^{*}, R\right) \backslash B\left(C^{*}, R-\varepsilon\right)\right) \cap K,
\end{aligned}
$$

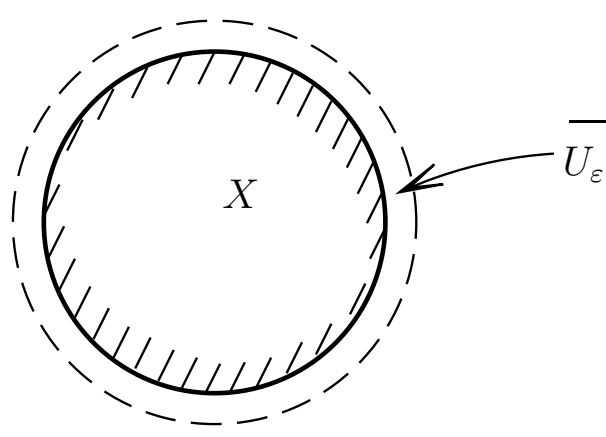

Figure 1. Example 1: the simplest convex set in the form of a disk.

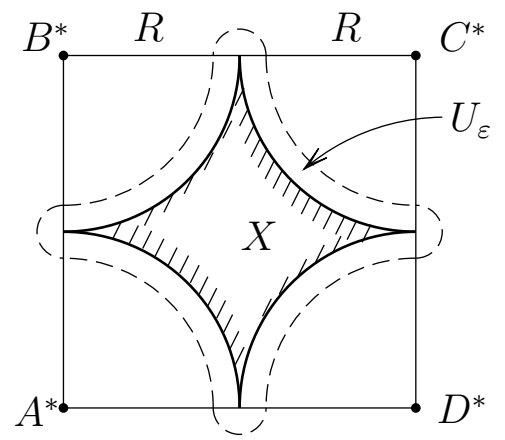

Figure 2. Example 2: a simple non-convex set. 


$$
U_{4}=\left(B\left(D^{*}, R\right) \backslash B\left(D^{*}, R-\varepsilon\right)\right) \cap K,
$$

where $B(a, r)=\left\{x=\left(x_{1}, x_{2}\right):\|x-a\| \leqslant r\right\}$ denotes the closed disk of radius $r>0$ centered at a point $a \in \mathbb{R}^{2}$.

Obviously, the total area of the four semidisks is $2 \pi \varepsilon^{2}$, and the sum of the areas of the curvilinear sets is

$$
s\left(U_{1}\right)+s\left(U_{2}\right)+s\left(U_{3}\right)+s\left(U_{4}\right)=\pi R^{2}-\pi(R-\varepsilon)^{2}=2 \pi R \varepsilon-\pi \varepsilon^{2} .
$$

As a result, we get

$$
s\left(U_{\varepsilon}\right)=2 \pi R \varepsilon+\pi \varepsilon^{2} .
$$

Since $l(\partial X)=2 \pi R$, equality (2.11) is satisfied in this case even though $X$ is a non-convex set.

Example 3. Consider another non-convex set $X=X_{1} \cup X_{1}$, (Fig. 3), where

$$
\begin{gathered}
X_{1}=\left\{x=\left(x_{1}, x_{2}\right): R \leqslant\left\|x-O_{1}\right\| \leqslant R+\mu, x_{2} \geqslant 0\right\}, \\
X_{2}=\left\{x=\left(x_{1}, x_{2}\right): R \leqslant\left\|x-O_{2}\right\| \leqslant R+\mu, x_{2} \leqslant 0\right\}, \\
O_{1}=(0,0), \quad O_{2}=(2 R+\mu, 0), \quad R>0, \quad \mu>0 .
\end{gathered}
$$

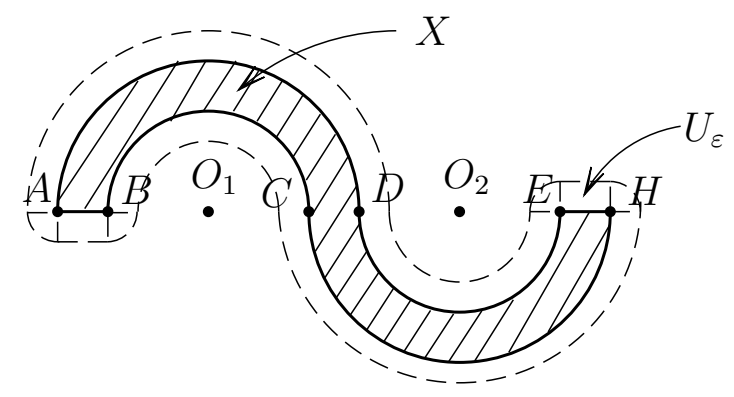

Figure 3. Example 3: a non-convex $S$-shaped set.

In this case, the $\varepsilon$-layer consists of four quarters of disk of radius $\varepsilon$ centered at the points $A$, $B, E$, and $H$, respectively, and four curvilinear sets adjacent to the $\operatorname{arcs} A D, B C, D E$, and $C H$.

It is easy to calculate that, firstly,

$$
l(\partial X)=2 \mu+2 \pi R+2 \pi(R+\mu)
$$

secondly,

$$
s\left(U_{\varepsilon}\right)=\pi \varepsilon^{2}+\pi(R+\mu+\varepsilon)^{2}-\pi(R+\mu)^{2}+\pi R^{2}-\pi(R-\varepsilon)^{2}=\pi \varepsilon^{2}+2 \pi(R+\mu) \varepsilon+2 \pi R \varepsilon .
$$

Thus, in this case, equality (2.11) holds despite the non-convexity of $X$.

Example 4. Let us give an example of a non-convex set $X$ for which equality (2.11) nevertheless turns into inequality (2.12). Let $X=K_{2} \backslash K_{1}$ (Fig. 4), where

$$
\begin{aligned}
& K_{1}=\left\{x=\left(x_{1}, x_{2}\right): \max \left\{\left|x_{1}\right|,\left|x_{2}\right|\right\} \leqslant 1\right\}, \\
& K_{2}=\left\{x=\left(x_{1}, x_{2}\right): \max \left\{\left|x_{1}\right|,\left|x_{2}\right|\right\} \leqslant 2\right\} .
\end{aligned}
$$

In this case, it is easy to calculate that

$$
l(\partial X)=8+4=12, \quad s\left(U_{\varepsilon}\right)=8 \varepsilon+\pi \varepsilon^{2}+4 \varepsilon-4 \varepsilon^{2}=12 \varepsilon-(4-\pi) \varepsilon^{2},
$$




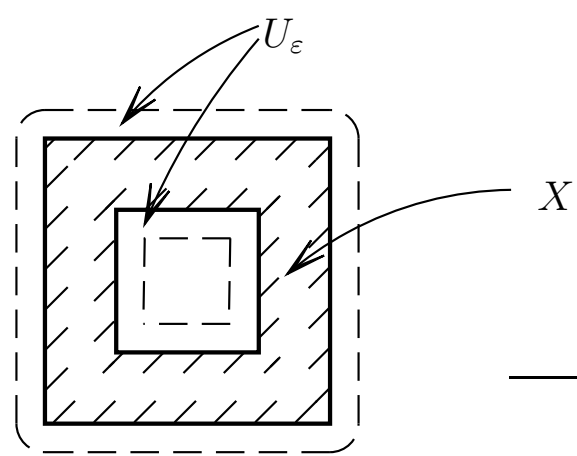

Figure 4. Example 4: a set with a cut-out square hole for which equality (2.11) is violated.

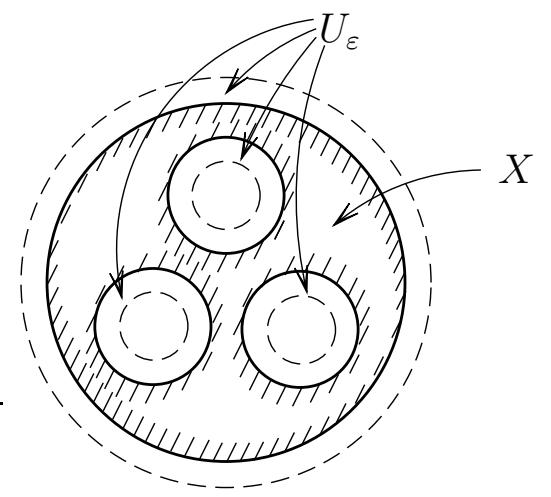

Figure 5. Example 5: a set with three holes for which equality (2.11) is violated to a large extent.

i.e., instead of equality (2.11), inequality (2.12) holds.

Example 5. Obviously, by increasing the number of "holes" inside the set $X$, one can increase the absolute value of the difference between $s\left(U_{\varepsilon}\right)$ and $l(\partial X) \cdot \varepsilon+\pi \varepsilon^{2}$. For example, the area of the $\varepsilon$-layer for the set $X$ shown in Fig. 5 (and consisting of a disk with three round holes)

$$
s\left(U_{\varepsilon}\right)=l(\partial X) \cdot \varepsilon-2 \pi \varepsilon^{2}
$$

regardless of the values of the radii of the disk and holes, provided that the radii of all holes are not less than $\varepsilon$.

Example 6. Note that the presence of "holes" inside $X$ is not necessary to violate equality (2.11). Fig. 6 shows a simply connected set $X$ for which

$$
s\left(U_{\varepsilon}\right)=l(\partial X) \cdot \varepsilon+5 \pi \varepsilon^{2}-16 \varepsilon^{2} .
$$

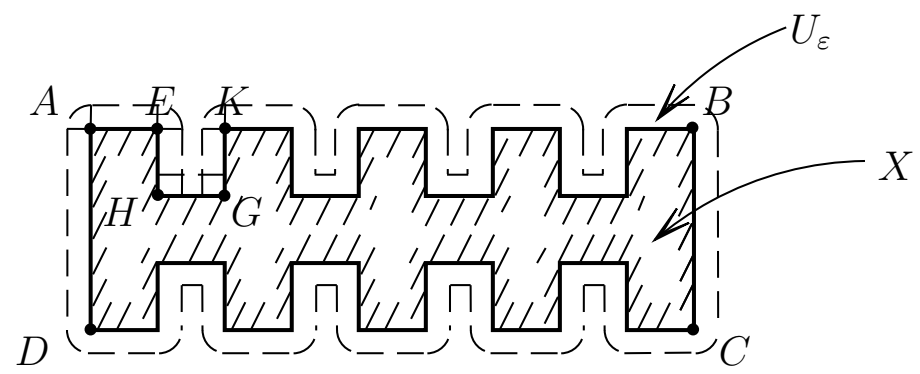

Figure 6. Example 6: a simply connected set with a negative quadratic addition in the formula for the $\varepsilon$-layer area.

Example \%. This example of the set $X$ consisting of two separate disks of radius $R$ (Fig. 7 ) shows that the connectedness condition for the set $X$ is necessary for inequality (2.12). Indeed, in this case,

$$
s\left(U_{\varepsilon}\right)=l(\partial X) \cdot \varepsilon+2 \pi \varepsilon^{2},
$$




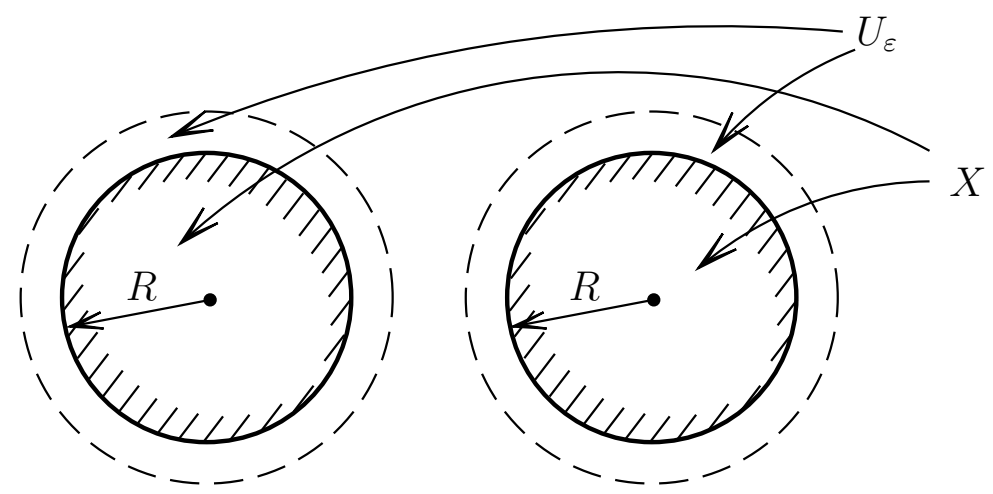

Figure 7. Example 7: a non-simply connected set for which inequality (2.12) is violated.

which violates inequality (2.12).

Example 8. In the last example, we will show that the addition to the main part $l(\partial X) \cdot \varepsilon$ in the expression for $s\left(U_{\varepsilon}\right)$ is not always proportional to $\varepsilon^{2}$. Indeed, let $X=B\left(O_{1}, R\right) \cup B\left(O_{2}, R\right)$, $0<\varepsilon<R$. In this case, the boundaries $\partial B\left(O_{1}, R\right)$ and $\partial B\left(O_{2}, R\right)$ (which are circles) intersect at two points $B$ and $D$. Define $\angle O_{1} O_{2} B=\varphi$ (Fig. 8).

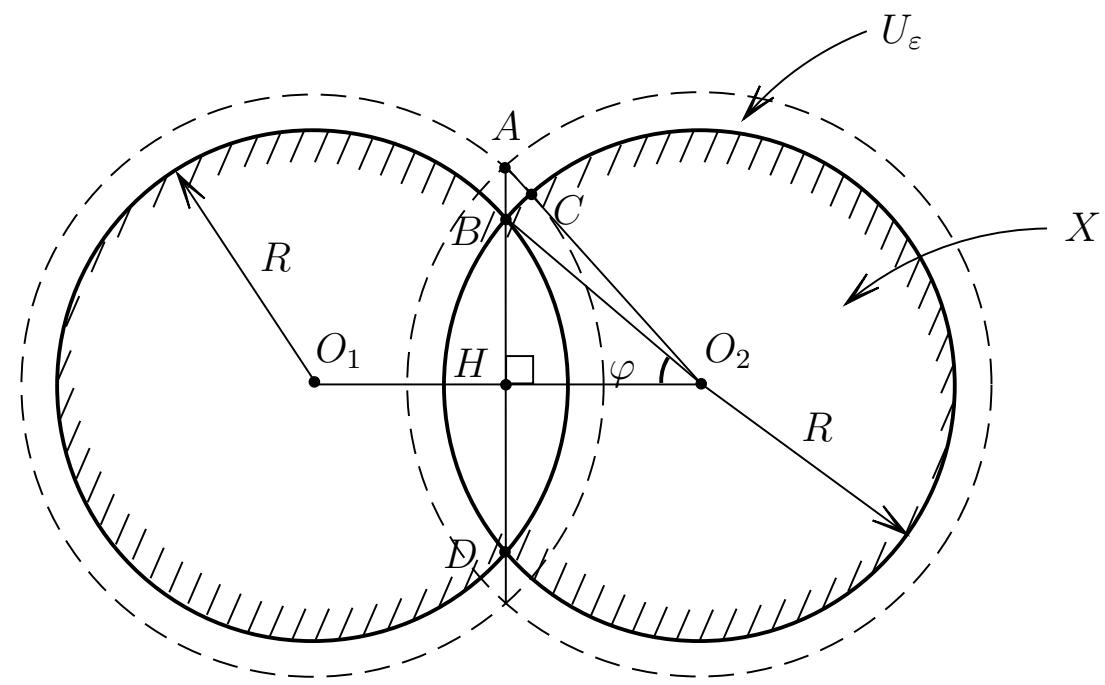

Figure 8. Example 8: two intersecting disks.

Then $l(\partial X)=4 R(\pi-\varphi)$.

From $\Delta \mathrm{BHO}_{2}$, we get $|\mathrm{BH}|=R \sin \varphi, \quad\left|\mathrm{HO}_{2}\right|=R \cos \varphi$.

Considering the right-angled triangle $\triangle A \mathrm{HO}_{2}$ and the Pythagorean theorem, we find that

$$
\left|A O_{2}\right|=R+\varepsilon, \quad|A H|=\sqrt{(R+\varepsilon)^{2}-R^{2} \cos ^{2} \varphi} .
$$

Further, the length of the segment $A B$ is

$$
|A B|=|A H|-|B H|=\sqrt{(R+\varepsilon)-R^{2} \cos ^{2} \varphi}-R \sin \varphi,
$$


the area of the triangle $\triangle A B O_{2}$ is

$$
s\left(\triangle A B O_{2}\right)=\frac{1}{2}|A B| \cdot\left|H O_{2}\right|=\frac{1}{2}\left(\sqrt{(R+\varepsilon)-R^{2} \cos ^{2} \varphi}-R \sin \varphi\right) R \cos \varphi,
$$

the value of the angle $\angle \mathrm{HO}_{2} \mathrm{~A}$ is

$$
\angle H O_{2} A=\arccos \frac{\left|H O_{2}\right|}{\left|A O_{2}\right|}=\arccos \left(\frac{R \cos \varphi}{R+\varepsilon}\right),
$$

and the value of the angle $\angle B O_{2} A$ is

$$
\angle B O_{2} A=\angle H O_{2} A-\varphi=\arccos \left(\frac{R \cos \varphi}{R+\varepsilon}\right)-\varphi .
$$

Consider the figure $A B C$ whose sides $A B$ and $A C \subset A O_{2}$ are segments and $B C$ is an arc of the circle centered at $\mathrm{O}_{2}$. Denote by $\mathrm{O}_{2} B C$ the sector based on the arc $B C$. The area of the figure $A B C$ is

$$
\begin{gathered}
s(A B C)=s\left(\triangle A B O_{2}\right)-s\left(O_{2} B C\right) \\
=\frac{1}{2}\left(\sqrt{(R+\varepsilon)^{2}-R^{2} \cos ^{2} \varphi}-R \sin \varphi\right) R \cos \varphi-\frac{1}{2} R^{2}\left(\arccos \left(\frac{R \cos \varphi}{R+\varepsilon}\right)-\varphi\right) .
\end{gathered}
$$

Taking into account the symmetry of the set $X$ about the lines $A D$ and $O_{1} O_{2}$, we obtain the area of the $\varepsilon$-layer:

$$
\begin{gathered}
s\left(U_{\varepsilon}\right)=4 s(A B C)+2 \cdot \frac{2 \pi-2 \varphi}{2}\left((R+\varepsilon)^{2}-R^{2}\right) \\
=2\left(\sqrt{(R+\varepsilon)^{2}-R^{2} \cos ^{2} \varphi}-R \sin \varphi\right) R \cos \varphi \\
+2 R^{2}\left(\varphi-\arccos \left(\frac{R \cos \varphi}{R+\varepsilon}\right)\right)+2\left(\pi-\arccos \left(\frac{R \cos \varphi}{R+\varepsilon}\right)\right)\left(2 R \varepsilon+\varepsilon^{2}\right)
\end{gathered}
$$

or

$$
\begin{gathered}
s\left(U_{\varepsilon}\right)=l(\partial X) \cdot \varepsilon+4\left(\varphi-\arccos \left(\frac{R \cos \varphi}{R+\varepsilon}\right)\right) R \varepsilon \\
+2\left(\sqrt{(R+\varepsilon)^{2}-R^{2} \cos ^{2} \varphi}-R \sin \varphi\right) R \cos \varphi \\
+2 R^{2}\left(\varphi-\arccos \left(\frac{R \cos \varphi}{R+\varepsilon}\right)\right)+2\left(\pi-\arccos \left(\frac{R \cos \varphi}{R+\varepsilon}\right)\right) \varepsilon^{2} .
\end{gathered}
$$

Note that, in the particular case $\varphi=0$,

$$
\begin{gathered}
s\left(U_{\varepsilon}\right)=l(\partial X) \cdot \varepsilon-4 R \arccos \left(\frac{R}{R+\varepsilon}\right) \varepsilon \\
+2\left(\sqrt{(R+\varepsilon)^{2}-R^{2}}\right) R-2 R^{2} \arccos \left(\frac{R}{R+\varepsilon}\right)+2\left(\pi-\arccos \left(\frac{R}{R+\varepsilon}\right)\right) \varepsilon^{2} \\
=l(\partial X) \cdot \varepsilon-\frac{8}{3} \sqrt{2 R} \cdot \varepsilon^{3 / 2}+2 \pi \varepsilon^{2}-\frac{14}{15} \frac{\sqrt{2} \varepsilon^{5 / 2}}{\sqrt{R}}+\frac{71}{420} \frac{\sqrt{2} \varepsilon^{7 / 2}}{R^{3 / 2}}+O\left(\varepsilon^{9 / 2}\right), \quad \varepsilon \rightarrow 0 .
\end{gathered}
$$

Thus, the addition to $l(\partial X)$ in the expression for $s\left(U_{\varepsilon}\right)$ may have rather complicated asymptotics and may not start with a term of the form $C \cdot \varepsilon^{2}$.

Remark 4. As can be seen from the examples, the question of the asymptotic behavior of the areas of the $\varepsilon$-layers $U_{\varepsilon}$ surrounding the set $X$ is nontrivial. It is related to questions of the geometric and topological structure of sets $X$ in $\mathbb{R}^{2}$ and has an independent meaning. 
In connection with Remark 3 , we introduce one more condition concerning the sets $X_{\alpha}\left(t_{i}, t_{0}, x\right)$ and $\widetilde{X}_{\beta}^{\Gamma}\left(t_{i}, t_{0}, y\right),(\alpha, x) \in \mathscr{L} \times X^{(0)},(\beta, y) \in \mathscr{L}^{(\rho)} \times X^{(\sigma)}, t_{i} \in \Gamma$.

E. The areas $s\left(U_{\alpha}\left(t_{i}\right)\right), s\left(\widetilde{U}_{\beta}^{\Gamma}\left(t_{i}\right)\right), \alpha, \beta \in \mathscr{L}, t_{i} \in \Gamma$, satisfy inequality $(2.12)$ for $\varepsilon=\varkappa^{\Delta}(\rho, \sigma)$ :

$$
\begin{aligned}
& s\left(U_{\alpha}\left(t_{i}\right)\right) \leqslant l\left(\partial X_{\alpha}\left(t_{i}, t_{0}, x\right)\right) \varepsilon+\pi \varepsilon^{2}, \\
& s\left(\widetilde{U}_{\beta}\left(t_{i}\right)\right) \leqslant l\left(\partial \widetilde{X}_{\beta}^{\Gamma}\left(t_{i}, t_{0}, y\right)\right) \varepsilon+\pi \varepsilon^{2} .
\end{aligned}
$$

Taking into account the definition of the sets $U_{\alpha}\left(\eta_{k}\right)$ and $\widetilde{U}_{\beta}^{\Gamma}\left(\eta_{k}\right), \eta_{k} \in \mathcal{T}$, and the condition $\mathbf{E}$, we obtain

$$
\begin{gathered}
\max \left(s\left(U_{\alpha}\left(\eta_{k}\right)\right), s\left(\widetilde{U}_{\beta}^{\Gamma}\left(\eta_{k}\right)\right)\right) \leqslant \max \left(l\left(\partial X_{\alpha}\left(\eta_{k}, t_{0}, x\right) \cap \Phi^{(k)}\right),\right. \\
\left.l\left(\partial \widetilde{X}_{\beta}^{\Gamma}\left(\eta_{k}, t_{0}, y\right) \cap \Phi^{(k)}\right)\right) \cdot \varkappa^{\Delta}(\rho, \sigma)+\pi \cdot \varkappa^{\Delta}(\rho, \sigma)^{2} \leqslant l^{*} \cdot \varkappa^{\Delta}(\rho, \sigma)+\pi \cdot \varkappa^{\Delta}(\rho, \sigma)^{2} .
\end{gathered}
$$

From (2.10) and (2.13), it follows that

$$
\left|s\left(X_{\alpha}\left(\eta_{k}, t_{0}, x\right) \cap \Phi^{(k)}\right)-s\left(\widetilde{X}_{\beta}^{\Gamma}\left(\eta_{k}, t_{0}, y\right) \cap \Phi^{(k)}\right)\right| \leqslant l^{*} \cdot \varkappa^{\Delta}(\rho, \sigma)+\pi \cdot \varkappa^{\Delta}(\rho, \sigma)^{2}, \quad \eta_{k} \in \mathcal{T} .
$$

From (2.14), it follows the estimate

$$
\left|s\left(\Phi^{(k)} \backslash X_{\alpha}\left(\eta_{k}, t_{0}, x\right)\right)-s\left(\Phi^{(k)} \backslash \tilde{X}_{\beta}^{\Gamma}\left(\eta_{k}, t_{0}, y\right)\right)\right| \leqslant l^{*} \cdot \varkappa^{\Delta}(\rho, \sigma)+\pi \cdot \varkappa^{\Delta}(\rho, \sigma)^{2}, \quad \eta_{k} \in \mathcal{T} .
$$

From (2.15), we obtain the estimate

$$
\left|\sum_{\eta_{k} \in \mathcal{T}} s\left(\Phi^{(k)} \backslash X_{\alpha}\left(\eta_{k}, t_{0}, x\right)\right)-\sum_{\eta_{k} \in \mathcal{T}} s\left(\Phi^{(k)} \backslash \widetilde{X}_{\beta}^{\Gamma}\left(\eta_{k}, t_{0}, y\right)\right)\right| \leqslant N_{*} \cdot\left(l^{*} \cdot \varkappa^{\Delta}(\rho, \sigma)+\pi \cdot \varkappa^{\Delta}(\rho, \sigma)^{2}\right),
$$

which can be written in the form

$$
\left|J^{(1)}(\alpha, x)-\widetilde{J}_{\Gamma}^{(1)}(\beta, y)\right| \leqslant N_{*} \cdot\left(l^{*} \cdot \varkappa^{\Delta}(\rho, \sigma)+\pi \cdot \varkappa^{\Delta}(\rho, \sigma)^{2}\right) .
$$

A similar scheme is used to derive the estimate

$$
\left|J^{(2)}(\alpha, x)-\widetilde{J}_{\Gamma}^{(2)}(\beta, y)\right| \leqslant l^{*} \cdot \varkappa^{\Delta}(\rho, \sigma)+\pi \cdot \varkappa^{\Delta}(\rho, \sigma)^{2} .
$$

From estimates (2.16) and (2.17), we obtain

$$
\left|J(\alpha, x)-\widetilde{J}_{\Gamma}(\beta, y)\right| \leqslant \zeta^{\Delta}(\rho, \sigma),
$$

where

$$
\zeta^{\Delta}(\rho, \sigma)=\left(N_{*}+1\right) \cdot\left(l^{*} \cdot \varkappa^{\Delta}(\rho, \sigma)+\pi \cdot \varkappa^{\Delta}(\rho, \sigma)^{2}\right) ;
$$

$\Delta, \rho$, and $\sigma$ are from $(0, \infty)$.

Based on esimate (2.18), we show that, for small $\Delta, \rho$, and $\sigma$, the solutions of Problems 2 and 3 are close, and we estimate this proximity.

Indeed, according to (2.18), the following inequality holds for every pair $(\beta, y) \in \mathscr{L}^{(\rho)} \times X^{(\sigma)} \subset$ $\mathscr{L} \times X^{(0)}$ :

$$
\left|J(\beta, y)-\widetilde{J}_{\Gamma}(\beta, y)\right| \leqslant \zeta^{\Delta}(\rho, \sigma),
$$

since the pair $(\beta, y) \in \mathscr{L} \times X^{(0)}$ is the closest pair in $\mathscr{L}^{(\rho)} \times X^{(\sigma)}$ to itself and, therefore, satisfies the inequalities $\|\beta-\beta\| \leqslant \rho$ and $\|y-y\| \leqslant \sigma$.

Hence, the following inequality holds for every pair $(\beta, y) \in \mathscr{L}^{(\rho)} \times X^{(\sigma)}$ :

$$
\widetilde{J}_{\Gamma}(\beta, y)-\zeta^{\Delta}(\rho, \sigma) \leqslant J(\beta, y) \leqslant \max _{(\alpha, x) \in \mathscr{L} \times X^{(0)}} J(\alpha, x),
$$


which implies

$$
\max _{(\beta, y) \in \mathscr{L}^{(\rho)} \times X^{(0)}} \widetilde{J}_{\Gamma}(\beta, y)-\zeta^{\Delta}(\rho, \sigma) \leqslant \max _{(\alpha, x) \in \mathscr{L} \times X^{(0)}} J(x, \alpha) .
$$

On the other hand, according to (2.18), the inequality

$$
J(\alpha, x) \leqslant \widetilde{J}_{\Gamma}(\beta, y)+\zeta^{\Delta}(\rho, \sigma)
$$

is true for every $(\alpha, x) \in \mathscr{L} \times X^{(0)}$ and $(\beta, y) \in \mathscr{L}^{(\rho)} \times X^{(\sigma)}$ such that $\|\alpha-\beta\| \leqslant \rho$ and $\|x-y\| \leqslant \sigma$.

Hence, for every pair $(\alpha, x) \in \mathscr{L} \times X^{(0)}$, the inequality

$$
J(\alpha, x) \leqslant \max _{(\beta, y) \in \mathscr{L}^{(\rho)} \times X^{(\sigma)}} \widetilde{J}_{\Gamma}(\beta, y)+\zeta^{\Delta}(\rho, \sigma)
$$

holds, which, in turn, implies

$$
\max _{(\alpha, x) \in \mathscr{L} \times X^{(0)}} J(\alpha, x) \leqslant \max _{(\beta, y) \in \mathscr{L}^{(\rho)} \times X^{(\sigma)}} \widetilde{J}_{\Gamma}(\beta, y)+\zeta^{\Delta}(\rho, \sigma) .
$$

Inequalities (2.19) and (2.20) imply

$$
\max _{(\beta, y) \in \mathscr{L}^{(\rho)} \times X^{(0)}} \widetilde{J}_{\Gamma}(\beta, y)-\zeta^{\Delta}(\rho, \sigma) \leqslant \max _{(\alpha, x) \in \mathscr{L} \times X^{(0)}} J(\alpha, x) \leqslant \max _{(\beta, y) \in \mathscr{L}^{(\rho)} \times X^{(\sigma)}} \widetilde{J}_{\Gamma}(\beta, y)+\zeta^{\Delta}(\rho, \sigma),
$$

i.e., we have the estimate

$$
\left|\max _{(\alpha, x) \in \mathscr{L} \times X^{(0)}} J(\alpha, x)-\max _{(\beta, y) \in \mathscr{L}^{(\rho)} \times X^{(0)}} \widetilde{J}_{\Gamma}(\beta, y)\right| \leqslant \zeta^{\Delta}(\rho, \sigma) .
$$

Let us say a pair $\left(\beta^{*}, y^{*}\right) \in \mathscr{L}^{(\rho)} \times X^{(\sigma)}$ is the optimal in the Problem 3, i.e.,

$$
\widetilde{J}_{\Gamma}\left(\beta^{*}, y^{*}\right)=\max _{(\beta, y) \in \mathscr{L}^{(\rho)} \times X^{(\sigma)}} \widetilde{J}_{\Gamma}(\beta, y) .
$$

Then we have the estimate

$$
\left|\max _{(\alpha, x) \in \mathscr{L} \times X^{(0)}} J(\alpha, x)-\widetilde{J}_{\Gamma}\left(\beta^{*}, y^{*}\right)\right| \leqslant \zeta^{\Delta}(\rho, \sigma) .
$$

In addition, as shown above, the pair $\left(\beta^{*}, y^{*}\right)$, like any pair $(\beta, y) \in \mathscr{L}^{(\rho)} \times X^{(\sigma)}$, satisfies the inequality

$$
\left|\widetilde{J}_{\Gamma}\left(\beta^{*}, y^{*}\right)-J\left(\beta^{*}, y^{*}\right)\right| \leqslant \zeta^{\Delta}(\rho, \sigma) .
$$

From (2.21) and (2.22), we obtain

$$
\left|\max _{(\alpha, x) \in \mathscr{L} \times X^{(0)}} J(\alpha, x)-J\left(\beta^{*}, y^{*}\right)\right| \leqslant 2 \zeta^{\Delta}(\rho, \sigma) .
$$

Inequality (2.23) states that every optimal pair $\left(\beta^{*}, y^{*}\right) \in \mathscr{L}^{(\rho)} \times X^{(\sigma)}$ for Problem 3 is $2 \zeta^{\Delta}(\rho, \sigma)$ optimal for Problem 2.

Taking into account the quadratic dependence of the function $\zeta^{\Delta}(\rho, \sigma)$ from the function $\varkappa^{\Delta}(\rho, \sigma)$ and the equality $\lim _{\Delta \downarrow 0, \rho \downarrow 0, \sigma \downarrow 0} \varkappa^{\Delta}(\rho, \sigma)=0$, we obtain $\lim _{\Delta \downarrow 0, \rho \downarrow 0, \sigma \downarrow 0} \zeta^{\Delta}(\rho, \sigma)=0$. Hence, for a predetermined $\varepsilon>0$, one can choose $\Delta=\Delta(\Gamma), \rho$, and $\sigma$ from $(0, \infty)$ so that the following inequality is true:

$$
\zeta^{\Delta}(\rho, \sigma) \leqslant \varepsilon
$$

Using $\rho$ and $\sigma$ satisfying (2.24), we can find a pair $\left(\beta^{*}, y^{*}\right) \in \mathscr{L}^{(\rho)} \times X^{(\sigma)}$ optimal for Problem 3 . As a result, the following statement is true. 
Theorem 3. Assume that the control system (1.1) in $\mathbb{R}^{2}$ satisfies the conditions $\boldsymbol{A}$ and $\boldsymbol{B}$ and, together with the partition $\Gamma=\left\{t_{0}, t_{1}, \ldots, t_{i}, \ldots, t_{N}=\vartheta\right\}\left(\Delta=\Delta(\Gamma)=N^{-1}\left(\vartheta-t_{0}\right)\right)$, the condition $\boldsymbol{C}$. Assume that, in Problems 2 and 3 , along with the conditions $\boldsymbol{A}, \boldsymbol{B}$, and $\boldsymbol{C}$, the conditions $\boldsymbol{D}$ and $\boldsymbol{E}$ are satisfied for the sets $X^{(0)}, X^{(\vartheta)}, \Phi_{k}=\Phi\left(\eta_{k}\right), X_{\alpha}\left(t_{i}, t_{0}, x\right)$, and $\widetilde{X}_{\beta}^{\Gamma}\left(t_{i}, t_{0}, y\right)$, where $(\alpha, x) \in \mathscr{L} \times X^{(0)},(\beta, y) \in \mathscr{L}^{(\rho)} \times X^{(\sigma)}, t_{i} \in \Gamma$, and $\eta_{k} \in \mathcal{T}$.

Then every optimal pair $\left(\beta^{*}, y^{*}\right) \in \mathscr{L}^{(\rho)} \times X^{(\sigma)}$ in Problem 3 is a $2 \zeta^{(\Delta)}(\rho, \sigma)$-optimal pair in Problem 2.

\section{Example}

In this section, we consider a nonlinear control system in $\mathbb{R}^{2}$ on the time interval $\left[t_{0}, \vartheta\right]=[0,1]$ depending on parameter $\alpha$ :

$$
\left\{\begin{array}{l}
\dot{x}_{1}=-x_{2} \cdot \frac{1}{2}\left(7+\frac{1}{4} \cos \left(x_{2}\right)+\frac{1}{2} \sin \left(\alpha_{1} t\right)\right)+\hat{a}(x) \frac{\|x\|}{1+\|x\|} u_{1}+0.1 \alpha_{1}, \\
\dot{x}_{2}=x_{1} \cdot \frac{1}{2}\left(7+\frac{1}{4} \cos \left(x_{1}\right)+\frac{1}{2} \sin \left(\alpha_{2} t\right)\right)+\hat{a}(x) \frac{\|x\|}{1+\|x\|} u_{2}+0.1 \alpha_{2}, \\
x(0) \in X^{(0)}
\end{array}\right.
$$

where

$$
\begin{gathered}
\hat{a}(x)=\left\{\begin{array}{c}
0.01 \quad \text { for } \quad\|x\|<1, \\
\frac{0.01}{\|x\|} \quad \text { for } \quad\|x\| \geqslant 1,
\end{array}\right. \\
\alpha=\left(\alpha_{1}, \alpha_{2}\right) \in I=\left[\frac{2}{3}, \frac{4}{3}\right] \times\left[-\frac{1}{3}, \frac{1}{3}\right], \\
u=\left(u_{1}, u_{2}\right) \in P=\left\{\bar{u}=\left(\bar{u}_{1}, \bar{u}_{2}\right):\|\bar{u}\| \leqslant 1\right\} .
\end{gathered}
$$

One problem of targeting the integral funnels of system (3.1) is formulated and solved in a soft setting close to Problem 3 from the previous section.

In this setting, the set $X^{(0)}$ of initial positions of system (3.1) is a closed set in $\mathbb{R}^{2}$ bounded by the Cassini oval

$$
\left(x^{2}+y^{2}\right)^{2}-2 c^{2}\left(x^{2}-y^{2}\right)^{2} \leqslant a^{4}-c^{4},
$$

where $a=4.4$ and $c=4$.

Along with $X^{(0)}$ in $\mathbb{R}^{2}$, the following two sets are also given:

(1) the rectangle $\Phi(t), t \in[0,1]$, with the initial set $\Phi\left(t_{0}\right)=[-9,-3] \times[-10,-6]$ rotating in one direction around its center $(-6,-8)$ over time 0.01 at the angle $1^{\circ}$;

(2) the ellipse

$$
X^{(\vartheta)}=\left\{\left(x_{1}, x_{2}\right) \in \mathbb{R}^{2}:\left(\frac{2}{5}\right)^{2}\left(x_{1}-12\right)^{2}+\left(\frac{2}{15}\right)^{2}\left(x_{2}+5\right)^{2} \leqslant 1\right\} .
$$

There are given the rectangle $\Phi(t), t \in[0,1]$, changing its orientation over time $t$, that we treat as a zone of dangerous stay during the entire period of time $[0,1]$, and the ellipse $X^{(\vartheta)}$ that we treat as a target set for the system (3.1) at the terminal time $\vartheta=1$.

We have the following two goals for the control system (3.1): 
(1) assuming that the reachable sets $X_{\alpha}\left(t, t_{0}, X^{(0)}\right), \alpha \in \mathscr{L}, t \in\left[t_{0}, \vartheta\right]$, can intersect the sets $\Phi(t)$, we must strive to ensure that the total intersection area $\Phi\left(\eta_{i}\right) \bigcap X_{\alpha}\left(\eta_{i}, t_{0}, X^{(0)}\right), \eta_{i} \in \mathscr{T}$, will be as small as possible; here $\mathscr{T}$ is some finite set in $\left[t_{0}, \vartheta\right]$;

(2) we must strive to ensure that the area of the intersection $X^{(\vartheta)} \cap X_{\alpha}\left(\vartheta, t_{0}, X^{(0)}\right)$ is as much as possible.

Let us formalize our targeting problem.

Introduce the notation

$$
\begin{gathered}
J^{(1)}(\alpha)=\sum_{\eta_{k} \in \mathscr{T}} s\left(\Phi\left(\eta_{k}\right) \backslash X_{\alpha}\left(\eta_{k}, t_{0}, X^{(0)}\right)\right), \\
J^{(2)}(\alpha)=\underset{\alpha \in \mathscr{L}}{s}\left(X^{(\vartheta)} \bigcap X_{\alpha}\left(\vartheta, t_{0}, X^{(0)}\right)\right), \\
J(\alpha)=\lambda_{1} J^{(1)}(\alpha)+\lambda_{2} J^{(2)}(\alpha),
\end{gathered}
$$

where $\lambda_{1}$ and $\lambda_{2}$ are from $[0,1], \lambda_{1}+\lambda_{2}=1$.

Problem 4 on targeting integral funnels of system (3.1) (soft setting). It is required to find $\alpha^{*} \in \mathscr{L}$ such that

$$
J\left(\alpha^{*}\right)=\max _{\alpha \in \mathscr{L}} J(\alpha)
$$

Remark 5. Problem 4 is close in setting to Problem 2 from Section 2 and differs from it by considering the sets $X_{\alpha}\left(t_{k}, t_{0}, X^{(0)}\right), \alpha \in \mathscr{L}$, with initial set $X^{(0)}$ instead of the sets $X_{\alpha}\left(t_{k}, t_{0}, x\right)$, $(\alpha, x) \in \mathscr{L} \times X^{(0)}$. This limitation will not affect the key estimates that we use in Problem 4 .

Since we cannot solve Problem 4 exactly, we formulate an approximation problem in which, instead of the set $\mathscr{L}$, there is a $\rho$-net $\mathscr{L}^{(\rho)}$. The partition $\Gamma$ is used as the interval $\left[t_{0}, \vartheta\right]$ and, instead of ideal reachable sets $X_{\alpha}\left(t, t_{0}, X^{(0)}\right)$, their approximations $\tilde{X}_{\alpha^{(r)}}^{\Gamma}\left(t_{i}, t_{0}, X^{(0)}\right), \alpha^{(r)} \in \mathscr{L}^{(\rho)}$, $t_{i} \in \Gamma$, are used.

Let us introduce the notation

$$
\begin{gathered}
\tilde{J}_{\Gamma}^{(1)}\left(\alpha^{(r)}\right)=\sum_{\eta_{i} \in \mathscr{T}} s\left(\Phi\left(\eta_{i}\right) \backslash \tilde{X}_{\alpha^{(r)}}^{\Gamma}\left(\eta_{i}, t_{0}, X^{(0)}\right)\right), \\
\tilde{J}_{\Gamma}^{(2)}\left(\alpha^{(r)}\right)=s\left(X^{(\vartheta)} \bigcap \tilde{X}_{\alpha^{(r)}}^{\Gamma}\left(\vartheta, t_{0}, X^{(0)}\right)\right) ;
\end{gathered}
$$

recall that $s(X)$ is the area of a set $X \subset \mathbb{R}^{2}$.

Let us clarify how we define the partition $\Gamma$ in the approximation problem and the $\rho$-net $\mathscr{L}^{(\rho)}$. Assume that

$$
\Gamma=\left\{t_{0}=0, t_{1}, \ldots, t_{i}, \ldots, t_{N}=\vartheta=1\right\},
$$

where $t_{i+1}-t_{i}=\Delta_{i}=\Delta=0.01$ and $N=100$;

$$
\mathscr{L}^{(\rho)}=\mathscr{L}_{1}^{(\rho)} \times \mathscr{L}_{2}^{(\rho)},
$$

where

$$
\begin{gathered}
\rho=\frac{\sqrt{2}}{15}, \\
\mathscr{L}_{1}^{(\rho)}=\left\{\alpha_{1}^{(l)} \in\left[\frac{2}{3}, \frac{4}{3}\right], \alpha_{1}^{(0)}=\frac{2}{3}, \alpha_{1}^{(l)}=l_{1}^{(0)}+\frac{l}{15}, l=\overline{1,10}\right\}, \\
\mathscr{L}_{2}^{(\rho)}=\left\{\alpha_{2}^{(k)} \in\left[-\frac{1}{3}, \frac{1}{3}\right], \alpha_{2}^{(0)}=-\frac{1}{3}, \alpha_{2}^{(k)}=l_{2}^{(0)}+\frac{k}{15}, k=\overline{1,10}\right\} ;
\end{gathered}
$$


in addition, points of the set $\mathscr{L}^{(\rho)}$ are parameterized by the parameter $r=\overline{1,121}$ and are denoted by $\alpha^{(r)}$.

Assume that

$$
\tilde{J}_{\Gamma}\left(\alpha^{(r)}\right)=\lambda_{1} \tilde{J}_{\Gamma}^{(1)}\left(\alpha^{(r)}\right)+\lambda_{2} \tilde{J}_{\Gamma}^{(2)}\left(\alpha^{(r)}\right),
$$

where $\lambda_{1}$ and $\lambda_{2}$ are defined above. Let us formulate an approximation problem.

Problem $4^{(a)}$. It is required to find $\alpha^{\left(r^{*}\right)} \in \mathscr{L}^{(\rho)}$ such that

$$
\tilde{J}_{\Gamma}\left(\alpha^{\left(r^{*}\right)}\right)=\max _{\alpha^{(r)} \in \mathscr{L}^{(\rho)}} \tilde{J}_{\Gamma}\left(\alpha^{(r)}\right) .
$$

It is important for us not only to calculate the optimal result $\tilde{J}_{\Gamma}\left(\alpha^{\left(r^{*}\right)}\right)$ in Problem $4^{(a)}$, but also find out how accurately it approximates the optimal result $J\left(\alpha^{*}\right)$ in Problem 4 . In other words, we are also interested in an upper estimate of the quantity $\left|J\left(\alpha^{*}\right)-\tilde{J}_{\Gamma}\left(\alpha^{\left(r^{*}\right)}\right)\right|$. Note that this estimate is completely analogous to estimate (2.18) from Section 2 with the only difference that here $\sigma=0$.

Let us calculate the numerical characteristics in Problem $4^{(a)}$ involved in an estimate of type (2.18). Some of them will turn out to be quite significant in size. This is connected both with the dynamics of system (3.1) and with the roughness of the approximations $\Gamma$ and $\mathscr{L}^{(\rho)}$ of the sets $\left[t_{0}, \vartheta\right]$ and $\mathscr{L}$.

The right-hand side of system (3.1) has the form

$$
f_{\alpha}(t, x, u)=\left(\begin{array}{c}
-\frac{x_{2}}{2} \cdot\left(7+\frac{1}{4} \cos \left(x_{2}\right)+\frac{1}{2} \sin \left(\alpha_{1} t\right)\right) \\
\frac{x_{1}}{2} \cdot\left(7+\frac{1}{4} \cos \left(x_{1}\right)+\frac{1}{2} \sin \left(\alpha_{2} t\right)\right)
\end{array}\right)+\hat{a}(x) \cdot \frac{\|x\|}{1+\|x\|} u+0.1 \alpha .
$$

Let us estimate from above the value $\left\|f_{\alpha}(t, x, u)\right\|$ :

$$
\begin{gathered}
\left\|f_{\alpha}(t, x, u)\right\| \\
\leqslant \sqrt{\frac{x_{2}^{2}}{4}\left(7+\frac{1}{4} \cos \left(x_{2}\right)+\frac{1}{2} \sin \left(\alpha_{1} t\right)\right)^{2}+\frac{x_{1}^{2}}{4}\left(7+\frac{1}{4} \cos \left(x_{1}\right)+\frac{1}{2} \sin \left(\alpha_{2} t\right)\right)^{2}} \\
+\hat{a}(x) \frac{\|x\|}{1+\|x\|}\|u\|+0.1\|\alpha\| \\
\leqslant \frac{31}{16}\|x\|+0.01 \cdot 1+0.1 \sqrt{\left(\frac{4}{3}\right)^{2}+\left(\frac{1}{3}\right)^{2}}<1.9375\|x\|+0.1475,
\end{gathered}
$$

i.e.,

$$
\left\|f_{\alpha}(t, x, u)\right\| \leqslant 1.9375\|x\|+0.1475 .
$$

Hence, under the condition B for system (3.1), we can set $\gamma=1.9375$.

Using the Cauchy-Bunyakovsky inequality and an inequality of the type $2 a b \leqslant a^{2}+b^{2}$, we estimate the variation of $\|x(t)\|^{2}$ along the trajectory $x=x(t)$ of system (3.1):

$$
\begin{gathered}
\frac{d\|x\|^{2}}{d t}=2\langle x, \dot{x}\rangle=2\left(x_{1} \dot{x}_{1}+x_{2} \dot{x}_{2}\right) \\
=-x_{1} x_{2}\left(7+\frac{1}{4} \cos x_{2}+\frac{1}{2} \sin \left(\alpha_{1} t\right)\right)+2 x_{1} \hat{\alpha}(x) \frac{\|x\|}{1+\|x\|} u_{1}+0.2 \alpha_{1} x_{1} \\
+x_{1} x_{2}\left(7+\frac{1}{4} \cos x_{1}+\frac{1}{2} \sin \left(\alpha_{2} t\right)\right)+2 x_{2} \hat{\alpha}(x) \frac{\|x\|}{1+\|x\|} u_{2}+0.2 \alpha_{2} x_{2} \\
=\frac{x_{1} x_{2}}{4}\left(\cos x_{1}-\cos x_{2}+2 \sin \left(\alpha_{2} t\right)-2 \sin \left(\alpha_{1} t\right)\right)+2\left(x_{1} u_{1}+x_{2} u_{2}\right) \hat{\alpha}(x) \frac{\|x\|}{1+\|x\|}+0.2\left(\alpha_{1} x_{2}+\alpha_{2} x_{2}\right)
\end{gathered}
$$




$$
\begin{gathered}
\leqslant \frac{6}{4}\left|x_{1} x_{2}\right|+2\|x\| \cdot\|u\| \hat{a}(x) \frac{\|x\|}{1+\|x\|}+0.2\|\alpha\| \cdot\|x\| \\
\leqslant \frac{3}{4}\|x\|^{2}+2 \hat{\alpha}(x)\|x\|+0.2\|x\| \sqrt{\left(\frac{4}{3}\right)^{2}+\left(\frac{1}{3}\right)^{2}} \\
\leqslant \frac{3}{4}\|x\|^{2}+0.295\|x\| \leqslant \frac{3}{4}\|x\|^{2}+\frac{3}{10}\|x\| .
\end{gathered}
$$

Given the equality

$$
\frac{d\|x\|^{2}}{d t}=2\|x\| \frac{d\|x\|}{d t}
$$

from the inequality

$$
\frac{d\|x\|^{2}}{d t} \leqslant \frac{3}{4}\|x\|^{2}+\frac{3}{10}\|x\|
$$

we get

$$
\frac{d\|x\|}{d t} \leqslant \frac{3}{8}\|x\|+\frac{3}{20} \quad \text { for } \quad\|x\| \neq 0 .
$$

From this inequality, we easily deduce the estimate

$$
\|x(t)\| \leqslant\left\|x\left(t_{0}\right)\right\| \cdot e^{3 / 8 \cdot\left(t-t_{0}\right)}+\frac{3 \cdot 8}{20 \cdot 3}\left(e^{3 / 8 \cdot\left(t-t_{0}\right)}-1\right), \quad t \in\left[t_{0}, \vartheta\right]=[0,1] .
$$

Hence,

$$
\max _{t \in\left[t_{0}, \vartheta\right]}\|x(t)\| \leqslant\left\|x\left(t_{0}\right)\right\| e^{3 / 8}+\frac{2}{5} \cdot\left(e^{3 / 8}-1\right) .
$$

Taking into account the equation for the Cassini oval, we obtain

$$
\max _{x\left(t_{0}\right) \in X^{(0)}}\left\|x\left(t_{0}\right)\right\|=\sqrt{a^{2}+c^{2}}=\sqrt{4^{2}+4.4^{2}} \approx 5.946 .
$$

From (3.3) and (3.4), we conclude that the following estimate holds for the trajectories $x(t)$, $x^{(0)} \in X^{(0)}$ of system $(3.1)$ :

$$
\max _{t \in\left[t_{0}, \vartheta\right]}\|x(t)\| \leqslant 8.833 .
$$

As constraints and a closed domain $D$ in the space of positions $(t, x)$ containing all possible motions $(t, x(t))$ of the control system together with some of their neighborhood (with respect to the phase variable), we can take the set

$$
D=\left\{(t, x): t \in\left[t_{0}, \vartheta\right],\|x\| \leqslant 8.833+\varepsilon\right\},
$$

where we set $\varepsilon=0.167$. In this case, we find that

$$
D=\{(t, x): t \in[0,1],\|x\| \leqslant 9\} .
$$

Estimate (3.2) and inclusion $(t, x) \in D$ imply

$$
\left\|f_{\alpha}(t, x, u)\right\| \leqslant 1.9375 \cdot\|x\|+0.1475 \leqslant 1.9375 \cdot 9+0.1475=17.585
$$

for $\alpha \in \mathscr{L}$ and $u \in P$.

It follows the inequality

$$
\max \left\{\|x(t)\|: t \in\left[t_{0}, \vartheta\right], x\left(t_{0}\right) \in X^{(0)}\right\} \leqslant 8.833 .
$$


The following inequality is valid for $(t, x, u)$ and $(t, y, u)$ from $D \times P$ and $\alpha \in \mathscr{L}$ :

$$
\begin{gathered}
\left\|f_{\alpha}(t, x, u)-f_{\alpha}(t, y, u)\right\| \\
\leqslant\left\|\left(\begin{array}{l}
\left(y_{2}-x_{2}\right) \\
\cdot \frac{1}{2}\left(7+\frac{1}{2} \sin \left(\alpha_{1} t\right)\right) \\
\left(x_{1}-y_{1}\right)
\end{array}\right)\right\|+\left\|\left(\begin{array}{l}
\frac{1}{2}\left(y_{2} \cos y_{2}-x_{2} \cos x_{2}\right) \\
\frac{1}{2}\left(x_{1} \cos x_{1}-y_{1} \sin \left(\alpha_{2} t\right)\right)
\end{array}\right)\right\| \\
+\left|\hat{\alpha}(x) \cdot \frac{\|x\|}{1+\|x\|}-\hat{\alpha}(y) \cdot \frac{\|y\|}{1+\|y\|}\right| \cdot\|u\| .
\end{gathered}
$$

Let us estimate from above each of the three terms on the right-hand side of this inequality.

We have

$$
\left\|\left(\begin{array}{l}
\left(y_{2}-x_{2}\right) \cdot \frac{1}{2}\left(7+\frac{1}{2} \sin \left(\alpha_{1} t\right)\right) \\
\left(x_{1}-y_{1}\right) \cdot \frac{1}{2}\left(7+\frac{1}{2} \sin \left(\alpha_{2} t\right)\right)
\end{array}\right)\right\| \leqslant \varphi(t)\|x-y\|,
$$

where

$$
\varphi(t)=\left\{\begin{array}{lll}
\frac{1}{2}+\frac{1}{2} \sin \left(\frac{4}{3} t\right) & \text { for } \quad t<\frac{3 \pi}{8}, \\
1 & \text { for } \quad t \geqslant \frac{3 \pi}{8} .
\end{array}\right.
$$

Further, taking into account the inequality

$$
\begin{gathered}
\left\|\frac{d\left(x_{k} \cos \left(x_{k}\right)\right)}{d x_{k}}\right\|=\left\|\cos \left(x_{k}\right)-x_{k} \sin \left(x_{k}\right)\right\| \leqslant \sqrt{1+x_{k}^{2}} \leqslant \sqrt{1+9^{2}} \approx 9.055, \\
\left(t, x_{k}\right) \in D, \quad k=1,2,
\end{gathered}
$$

we get

$$
\left\|\left(\begin{array}{l}
\frac{1}{8}\left(y_{2} \cos y_{2}-x_{2} \cos x_{2}\right) \\
\frac{1}{8}\left(x_{1} \cos x_{1}-y_{1} \cos y_{1}\right)
\end{array}\right)\right\| \leqslant \frac{9.055}{8}\|x-y\| .
$$

Let us now estimate the third term. To do this, we introduce

$$
R=\hat{\alpha}(x) \cdot \frac{\|x\|}{1+\|x\|}-\hat{\alpha}(y) \cdot \frac{\|y\|}{1+\|y\|}
$$

and estimate $|R|$ :

$$
|R|=\left|\frac{\hat{\alpha}(x)\|x\|(1+\|y\|)-\hat{\alpha}(y)\|y\|(1+\|x\|)}{(1+\|x\|) \cdot(1+\|y\|)}\right| \leqslant|\hat{\alpha}(x)\|x\|-\hat{\alpha}(y)\|y\|+\|x\|\|y\|(\hat{\alpha}(x)-\hat{\alpha}(y))| .
$$

We consider four cases for further estimation of $|R|$.

Case 1. $\|x\|<1$ and $\|y\|<1$. Then

$$
\begin{gathered}
\hat{\alpha}(x)=\hat{\alpha}(y)=0.01, \\
|R| \leqslant 0.01\|\mid x\|-\|y\| .
\end{gathered}
$$

Case 2. $\|x\| \geqslant 1$ and $\|y\| \geqslant 1$. Then

$$
\begin{gathered}
\hat{\alpha}(x)=\frac{0.01}{\|x\|}, \quad \hat{\alpha}(y)=\frac{0.01}{\|y\|}, \\
|R| \leqslant|0.01(\|y\|-\|x\|)|=0.01\||x\|-\| y \|| .
\end{gathered}
$$


Case 3. $\|x\|<1$ and $\|y\| \geqslant 1$. Then

$$
\begin{gathered}
\hat{\alpha}(x)=0.01, \quad \hat{\alpha}(y)=\frac{0.01}{\|y\|} \\
|R| \leqslant\left|0.01\|x\|-\frac{0.01}{\|y\|} \cdot\|y\|+\|x\| \cdot\|y\|\left(0.01-\frac{0.01}{\|y\|}\right)\right|=0.01 \cdot|1-\|x\| \cdot\|y\|| .
\end{gathered}
$$

Consider two subcases.

(a) $\|x\| \cdot\|y\| \geqslant 1$. Then

$$
|R| \leqslant 0.01|1-\|x\| \cdot\|y\|| \leqslant 0.01|1-\|y\|| \leqslant 0.01\||| x\|-\|y\| \mid<0.01\|x-y\| .
$$

(b) $\|x\| \cdot\|y\|<1$. Then

$$
|R| \leqslant 0.01|1-\|x\|\|y\|| \leqslant 0.01|1-\|x\|| \leqslant 0.01\||y\|-\| x\|\mid \leqslant 0.01\| y-x \| .
$$

Case 4. $\|x\| \geqslant 1$ and $\|y\|<1$. Since this case is similar to Case 3 , we have the inequality $|R| \leqslant 0.01\|x-y\|$.

Thus, in all cases, we have the inequality $|R| \leqslant 0.01\|x-y\|$.

Taking this inequality into account, we obtain an estimate for the third term:

$$
|R| \cdot\|u\| \leqslant 0.01 \cdot\|x-y\| .
$$

As a result, for $(t, x, u)$ and $(t, y, u)$ from $D \times P$ and $\alpha \in \mathscr{L}$, we get

$$
\left\|f_{\alpha}(t, x, u)-f_{\alpha}(t, y, u)\right\| \leqslant L(t)\|x-y\|,
$$

where $L(t)=\varphi(t)+1.142$, and we can take $L=2.142$.

Let us now estimate from above the value

$$
\left\|f_{\alpha}(t, x, u)-f_{\beta}(\tau, x, u)\right\|,
$$

where $(t, x, u)$ and $(\tau, x, u)$ from $D \times P$ and $\alpha$ and $\beta$ are from $\mathscr{L}$ :

$$
\begin{gathered}
\left\|f_{\alpha}(t, x, u)-f_{\beta}(\tau, x, u)\right\| \leqslant\left\|\left(\begin{array}{c}
-\frac{1}{4} x_{2} \cdot\left(\sin \left(\alpha_{1} t\right)-\sin \left(\beta_{1} \tau\right)\right) \\
\frac{1}{4} x_{1} \cdot\left(\sin \left(\alpha_{2} t\right)-\sin \left(\beta_{2} \tau\right)\right)
\end{array}\right)\right\|+0.1\left\|\left(\begin{array}{c}
\alpha_{1}-\beta_{1} \\
\alpha_{2}-\beta_{2}
\end{array}\right)\right\| \\
=\frac{1}{4} \sqrt{x_{2}^{2} \cdot\left(\sin \left(\alpha_{1} t\right)-\sin \left(\beta_{1} \tau\right)\right)^{2}+x_{1}^{2} \cdot\left(\sin \left(\alpha_{2} t\right)-\sin \left(\beta_{2} \tau\right)\right)^{2}}+0.1\|\alpha-\beta\| \\
\leqslant \frac{1}{4}\|x\| \sqrt{\left(\alpha_{1} t-\beta_{1} \tau\right)^{2}+\left(\alpha_{2} t-\beta_{2} \tau\right)^{2}}+0.1\|\alpha-\beta\| \\
\leqslant \frac{1}{4}\|x\|\left(\left|\alpha_{1} t-\beta_{1} \tau\right|+\left|\alpha_{2} t-\beta_{2} \tau\right|\right)+0.1\|\alpha-\beta\| \\
=\frac{1}{4}\|x\|\left(\left|\alpha_{1} t-\alpha_{1} \tau+\alpha_{1} \tau-\beta_{1} \tau\right|+\left|\alpha_{2} t-\alpha_{2} \tau+\alpha_{2} \tau-\beta_{2} \tau\right|\right)+0.1\|\alpha-\beta\| \\
\leqslant \frac{1}{4}\|x\|\left(\alpha_{1}|t-\tau|+\tau\left|\alpha_{1}-\beta_{1}\right|+\alpha_{2}|t-\tau|+\tau\left|\alpha_{2}-\beta_{2}\right|\right)+0.1\|\alpha-\beta\| \\
\leqslant \frac{1}{4}\|x\|\left(\left(\alpha_{1}+\alpha_{2}\right)|t-\tau|+\tau\left(\left|\alpha_{1}-\beta_{1}\right|+\left|\alpha_{2}-\beta_{2}\right|\right)\right)+0.1\|\alpha-\beta\| \\
\leqslant \frac{1}{4}\|x\|\left(\frac{5}{3}|t-\tau|+\tau \sqrt{2}\|\alpha-\beta\|\right)+0.1\|\alpha-\beta\|
\end{gathered}
$$




$$
\begin{gathered}
\leqslant \frac{1}{4} \max _{(t, x) \in D}\|x\| \cdot\left(\frac{5}{3}|t-\tau|+\sqrt{2}\|\alpha-\beta\|\right)+0.1\|\alpha-\beta\| \\
\leqslant \frac{1}{4} \max _{(t, x) \in D}\|x\| \cdot \max \left(\frac{5}{3}, \sqrt{2}+\frac{0.2}{\max _{(t, x) \in D}\|x\|}\right) \cdot(|t-\tau|+\|\alpha-\beta\|) \\
=\frac{1}{4} \cdot 9 \cdot \frac{5}{3} \cdot(|t-\tau|+\|\alpha-\beta\|)=\frac{15}{4} \cdot(|t-\tau|+\|\alpha-\beta\|) .
\end{gathered}
$$

As a result, we obtain the following estimate for $(t, x, u)$ and $(\tau, x, u)$ from $D \times P$ and $\alpha$ and $\beta$ from $\mathscr{L}$ :

$$
\left\|f_{\alpha}(t, x, u)-f_{\beta}(\tau, x, u)\right\| \leqslant \frac{15}{4}(|t-\tau|+\|\alpha-\beta\|),
$$

from which it follows that, in the problem under consideration, we can take

$$
\omega^{*}(\xi)=\frac{15}{4} \xi, \quad \xi \in(0, \infty) .
$$

So, we have calculated the main characteristics involved in this problem in an estimate of the type of estimate (2.18): $K, L(t), t \in[0,1], L \in(0, \infty)$, and $\omega^{*}(\xi), \xi \in(0, \infty)$. Let us supplement them with several more characteristics participating in this estimate. Namely, the performed calculations show that the lengths of the boundaries of the sets $X^{(0)}, X^{(\vartheta)}, \Phi\left(t_{i}\right), t_{i} \in \Gamma$, and $\tilde{X}_{\alpha^{(r)}}^{\Gamma}\left(t_{i}, t_{0}, X^{(0)}\right), t_{i} \in \Gamma, \alpha^{(r)} \in \mathscr{L}^{(\rho)}$, can be etsimated by the number $l^{*}=32$. We also assume that $\Delta=\Delta(\Gamma)=\Delta_{i}=t_{i+1}-t_{i}=1 / N=0.01$, where $N=100, N_{*}=N, \rho=1 / 15$, and $\sigma=0$ as noted above.

Having determined all the main numerical characteristics in the considered problem, we proceed to calculating the main estimate of the type of estimate (2.18).

The following relations are valid:

$$
\begin{gathered}
\varkappa^{\Delta}(\rho, \sigma)=e^{\sum_{k=0}^{N-1} L\left(t_{k}\right) \Delta_{k}} \cdot\left(\left(\vartheta-t_{0}\right) \cdot \omega^{*}(\rho)+2\left(\vartheta-t_{0}\right) \omega^{*}(\Delta)+2 K \sum_{k=0}^{N-1} L\left(t_{k}\right) \Delta_{k}+\sigma\right) \\
\approx e^{1.926} \cdot\left(\frac{15}{4} \rho+2 \cdot \frac{15}{4} \Delta+2 \cdot 17.585 \Delta \cdot 1.926\right) \approx 516.3 \cdot \Delta+25.73 \cdot \rho \\
\zeta^{\Delta}(\rho, \sigma)=\left(N_{*}+1\right) \cdot\left(l^{*} \cdot \varkappa^{\Delta}(\rho, \sigma)+\pi \varkappa^{\Delta}(\rho, \sigma)^{2}\right) \\
\approx(N+1) \cdot\left(32 \cdot(516.3 \cdot \Delta+25.73 \cdot \rho)+3.142 \cdot(516.3 \cdot \Delta+25.73 \cdot \rho)^{2}\right) \approx 37242.74 .
\end{gathered}
$$

Hence, we obtain the following estimate of the mismatch of the optimal results $J\left(\alpha^{*}\right)$ and $\tilde{J}_{\Gamma}\left(\alpha_{r}^{*}\right)$ in Problems 4 and $4^{(a)}$ :

$$
\left|J\left(\alpha^{*}\right)-\widetilde{J}_{\Gamma}\left(\alpha^{r^{*}}\right)\right| \leqslant \zeta^{\Delta}(\rho, \sigma) \approx 37242.74
$$

Remark 6. Estimating the mismatch between the optimal results in Problems 4 and $4^{(a)}$, we found that estimate (3.5) is very rough. The roughness of estimate (3.5) is due to several factors:

(1) the dynamics of system (3.1);

(2) the presence of exponential quantities in the derivation of an estimate, which is standard for control problems with a Lipschitz right-hand side of the control system with a phase variable;

(3) the roughness of our discrete approximations $\mathscr{L}^{(\rho)}$ and $\Gamma$ of the compact set $\mathscr{L}$ and the interval $[0,1]$ due to the limited capabilities of computer technology. 
Note that, although estimate (3.5) is rough, it was obtained within the framework of the theory developed in Sections 1 and 2 and does not reflect the real value of the mismatch $\left|J\left(\alpha^{*}\right)-\widetilde{J}_{\Gamma}\left(\alpha^{r^{*}}\right)\right|$, which is much smaller.

Nevertheless, the question arises, how and by what means can estimate (3.5) be improved. One way to improve this is to establish more accurate approximations $\mathscr{L}^{(\rho)}$ and $\Gamma$ for the compact set $\mathscr{L}$ and the time interval $\left[t_{0}, \vartheta\right]=[0,1]$. In addition, if in the setting of Problems 4 and $4^{(a)}$, the number $N_{*}$ is small, then it also improves estimate (3.5). Then

As an example, let us set $\rho=1 / 150, N_{*}=1, N=1000$, and therefore $\Delta=\Delta(\Gamma)=0.001$.

$$
\begin{gathered}
\varkappa^{\Delta}(\rho, \sigma)=e^{\sum_{k=0}^{N-1} L\left(\eta_{k}\right) \Delta_{k}} \cdot\left(\left(\vartheta-t_{0}\right) \cdot \omega^{*}(\rho)+2\left(\vartheta-t_{0}\right) \omega^{*}(\Delta)+2 K \Delta \sum_{k=0}^{N-1} L\left(\eta_{k}\right) \Delta_{k}\right) \\
\approx e^{1.928}\left(\frac{15}{4} \rho+2 \cdot \frac{15}{4} \Delta+2 \cdot 17.585 \cdot \Delta \cdot 1.928\right) \approx 517.8 \cdot \Delta+25.78 \cdot \rho \\
\zeta^{\Delta}(\rho, \sigma)=\left(N_{*}+1\right) \cdot\left(l^{*} \cdot \varkappa^{\Delta}(\rho, \sigma)+\pi \varkappa^{\Delta}(\rho, \sigma)^{2}\right) \\
\approx 2 \cdot\left(32 \cdot(517.8 \Delta+25.78 \rho)+3.142(517.8 \cdot \Delta+25.78 \cdot \rho)^{2}\right) \approx 47.13 .
\end{gathered}
$$

We see that the decrease in the values $\rho$ and $\Delta$ by a factor of 10 and the number $N_{*}$ by a factor of 100 led to a significant improvement in the value of $\zeta^{\Delta}(\rho, \sigma)$.

Note also that if the compact set $\mathscr{L}$ is finite by the statement of Problem $4^{(a)}$, then we can treat it as a finite approximation $\mathscr{L}^{(\rho)}$ of itself with the value $\rho=0$.

In this case, with the same $N_{*}$ and $N$ as in the previous example, we get the estimate

$$
\left|J\left(\alpha^{*}\right)-\widetilde{J}_{\Gamma}\left(\alpha^{r^{*}}\right)\right| \leqslant \zeta^{\Delta}(\rho, \sigma) \approx 34.82 .
$$

For the example under consideration, we considered three variants of Problem 2 on targeting integral funnels (in a soft setting). Moreover, the peculiarity of our consideration is that we do not vary the starting point $x^{(0)}$ in the set $X^{(0)}$ and, instead of the sets $\widetilde{X}_{\alpha}^{\Gamma}\left(t_{i}, t_{0}, x^{(0)}\right)$, consider the reachable sets $\widetilde{X}_{\alpha}^{\Gamma}\left(t_{i}, t_{0}, X^{(0)}\right), \alpha \in \mathscr{L}$.

Each of the variants is determined by the choice of a pair of numbers $\lambda_{1}$ and $\lambda_{2}\left(\lambda_{1}, \lambda_{2} \in[0,1]\right.$, $\left.\lambda_{1}+\lambda_{2}=1\right)$ :

Variant 1. $\lambda_{1}=0.1$ and $\lambda_{2}=0.9$;

Variant 2. $\lambda_{1}=0.5$ and $\lambda_{2}=0.5$;

Variant 3. $\lambda_{1}=0.9$ and $\lambda_{2}=0.1$.

For each of the variants, in the set $\mathscr{L}^{(\rho)} \subset \mathscr{L}$, the optimal point $\alpha^{*}=\left(\alpha_{1}^{*}, \alpha_{2}^{*}\right)$ is calculated:

Variant 1. $\alpha_{1}^{*}=0.66667$ and $\alpha_{2}^{*}=0.33333$;

Variant 2. $\alpha_{1}^{*}=0.80000$ and $\alpha_{2}^{*}=0.33333$;

Variant 3. $\alpha_{1}^{*}=1.26670$ and $\alpha_{2}^{*}=0.33333$.

Each of the three options is illustrated with six figures (Fig. 9-Fig. 14, Fig. 15-Fig. 20, Fig. 21Fig. 26) that correspond to the times $t_{i}=0 ; 0.2 ; 0.4 ; 0.6 ; 0.8 ; 1.0$ of the partition $\Gamma$. The figures show the sets $\widetilde{X}_{\alpha^{*}}^{\Gamma}\left(t_{i}, t_{0}, X^{(0)}\right)$ and $\Phi\left(t_{i}\right)$ corresponding to these numbers and the target set $M$.

Also, each of the three variants indicates the optimal result obtained in the course of an approximate solution of Problem 2:

Variant 1. $J\left(\alpha^{*}, X^{(0)}\right)=38.4361$;

Variant 2. $J\left(\alpha^{*}, X^{(0)}\right)=743.9625$;

Variant 3. $J\left(\alpha^{*}, X^{(0)}\right)=2450$. 
$\underline{\text { Variant } 1}$

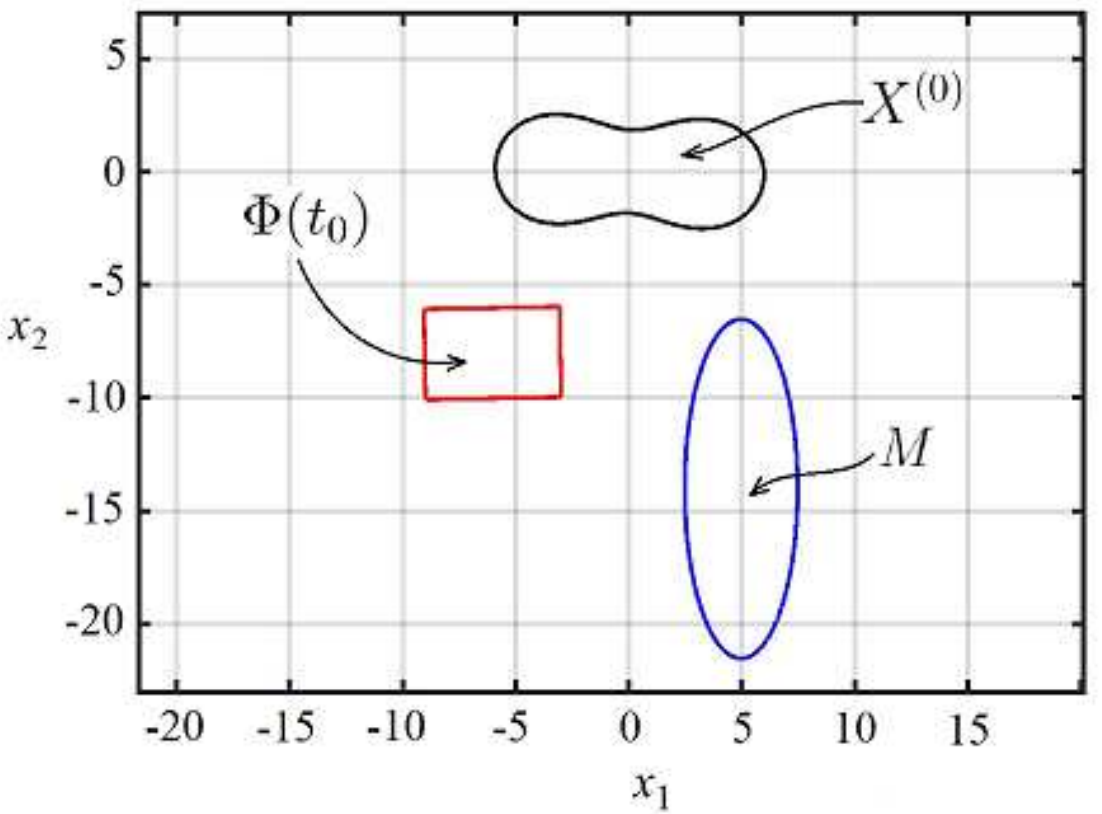

Figure 9. The sets $M, \Phi\left(t_{i}\right)$, and $\widetilde{X}_{\alpha}^{\Gamma}\left(t_{i}, t_{0}, X^{(0)}\right)$ for $\alpha_{1}^{*}=0.66667, \alpha_{2}^{*}=0.33333$, and $t_{i}=0$.

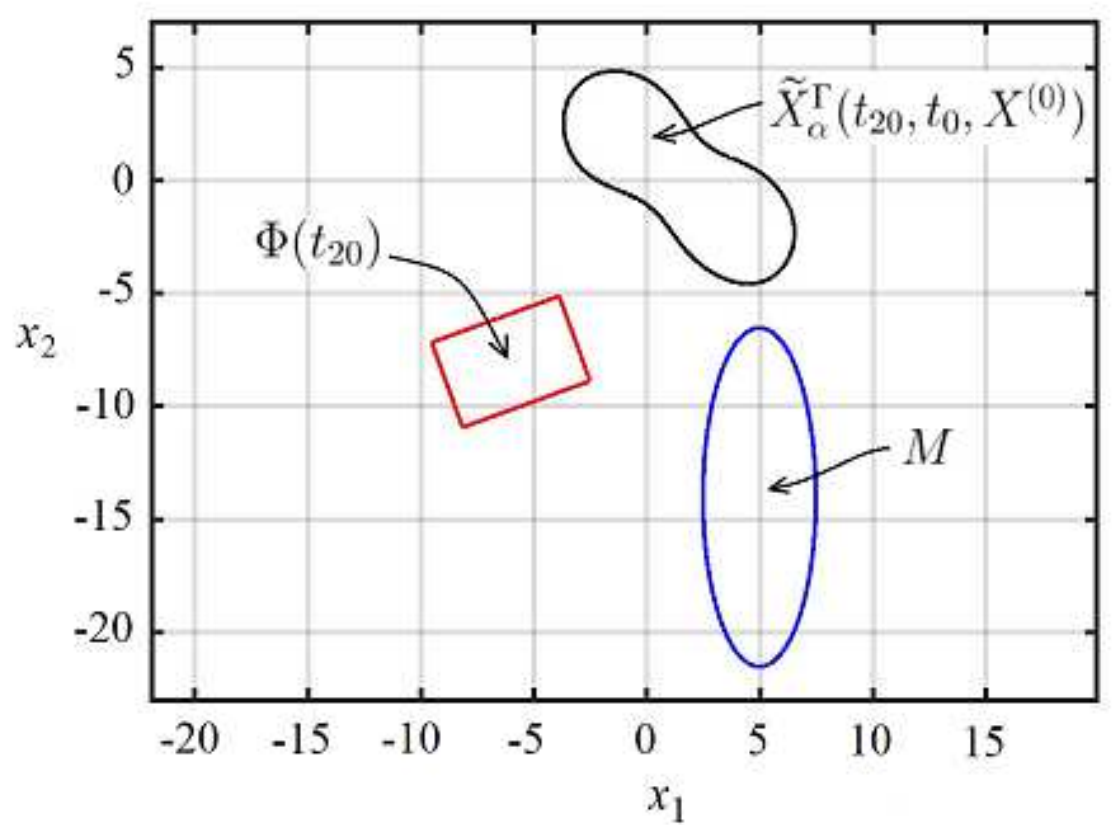

Figure 10. The sets $M, \Phi\left(t_{i}\right)$, and $\widetilde{X}_{\alpha}^{\Gamma}\left(t_{i}, t_{0}, X^{(0)}\right)$ for $\alpha_{1}^{*}=0.66667, \alpha_{2}^{*}=0.33333$, and $t_{i}=0.2$. 


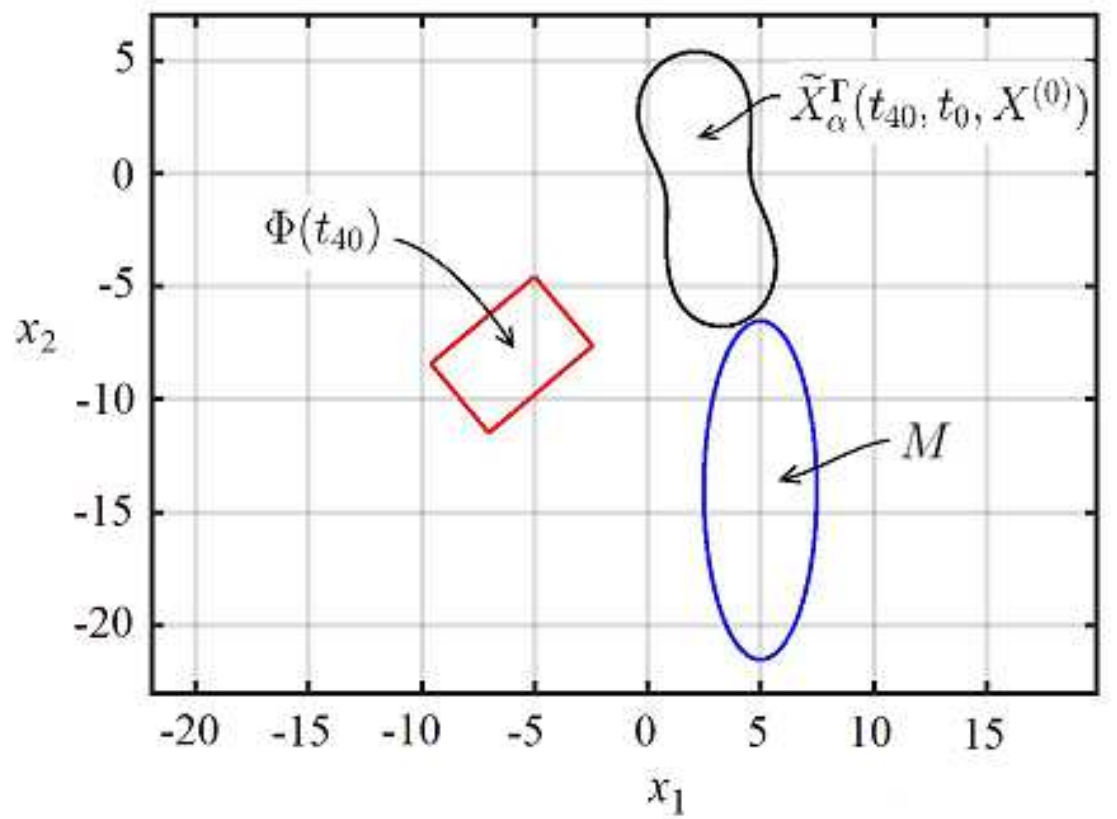

Figure 11. The sets $M, \Phi\left(t_{i}\right)$, and $\widetilde{X}_{\alpha}^{\Gamma}\left(t_{i}, t_{0}, X^{(0)}\right)$ for $\alpha_{1}^{*}=0.66667, \alpha_{2}^{*}=0.33333$, and $t_{i}=0.4$.

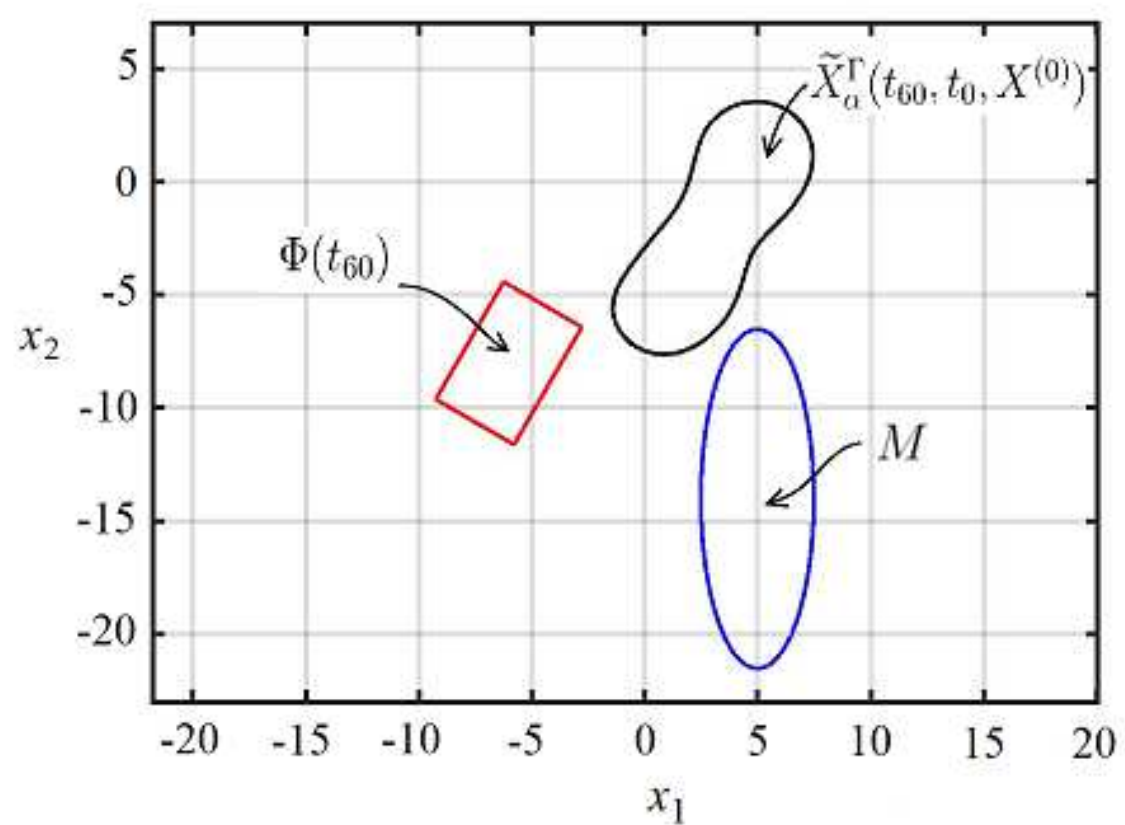

Figure 12. The sets $M, \Phi\left(t_{i}\right)$, and $\widetilde{X}_{\alpha}^{\Gamma}\left(t_{i}, t_{0}, X^{(0)}\right)$ for $\alpha_{1}^{*}=0.66667, \alpha_{2}^{*}=0.33333$, and $t_{i}=0.6$. 


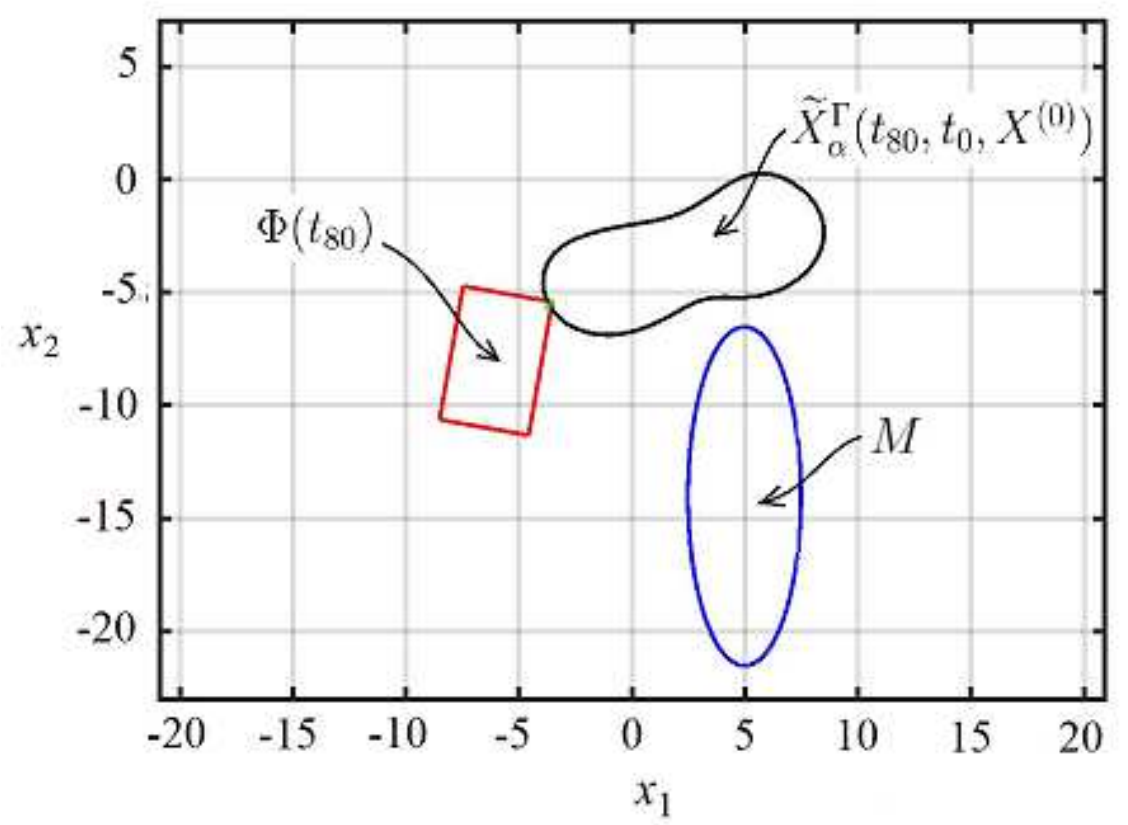

Figure 13. The sets $M, \Phi\left(t_{i}\right)$, and $\widetilde{X}_{\alpha}^{\Gamma}\left(t_{i}, t_{0}, X^{(0)}\right)$ for $\alpha_{1}^{*}=0.66667, \alpha_{2}^{*}=0.33333$, and $t_{i}=0.8$.

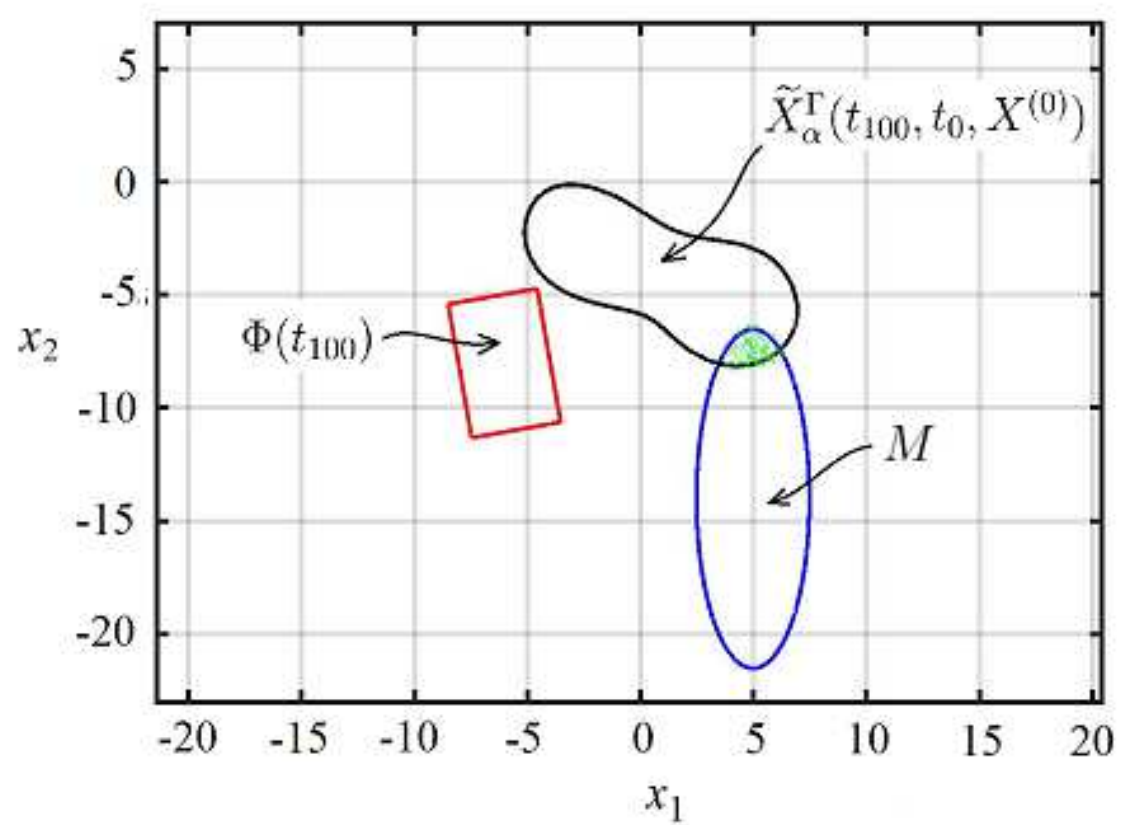

Figure 14. The sets $M, \Phi\left(t_{i}\right)$, and $\widetilde{X}_{\alpha}^{\Gamma}\left(t_{i}, t_{0}, X^{(0)}\right)$ for $\alpha_{1}^{*}=0.66667, \alpha_{2}^{*}=0.33333$, and $t_{i}=1$. 
$\underline{\text { Variant } 2}$

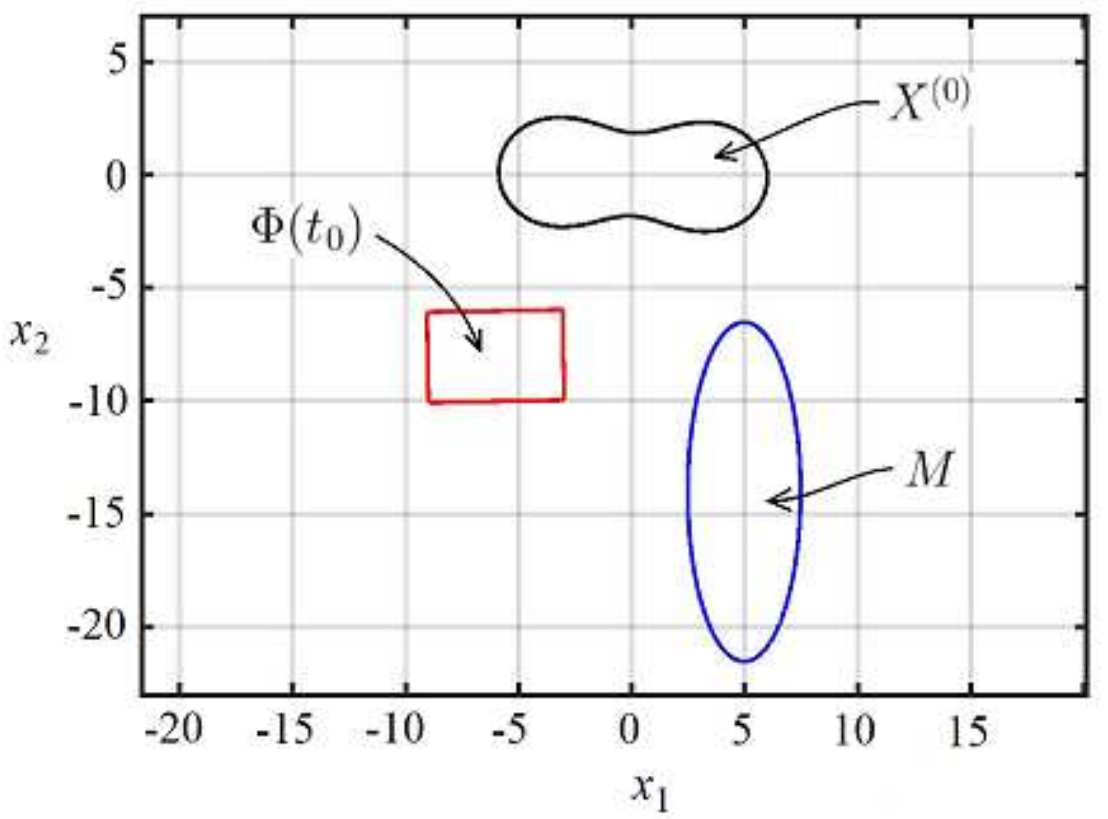

Figure 15. The sets $M, \Phi\left(t_{i}\right)$, and $\widetilde{X}_{\alpha}^{\Gamma}\left(t_{i}, t_{0}, X^{(0)}\right)$ for $\alpha_{1}^{*}=0.8, \alpha_{2}^{*}=0.33333$, and $t_{i}=0$.

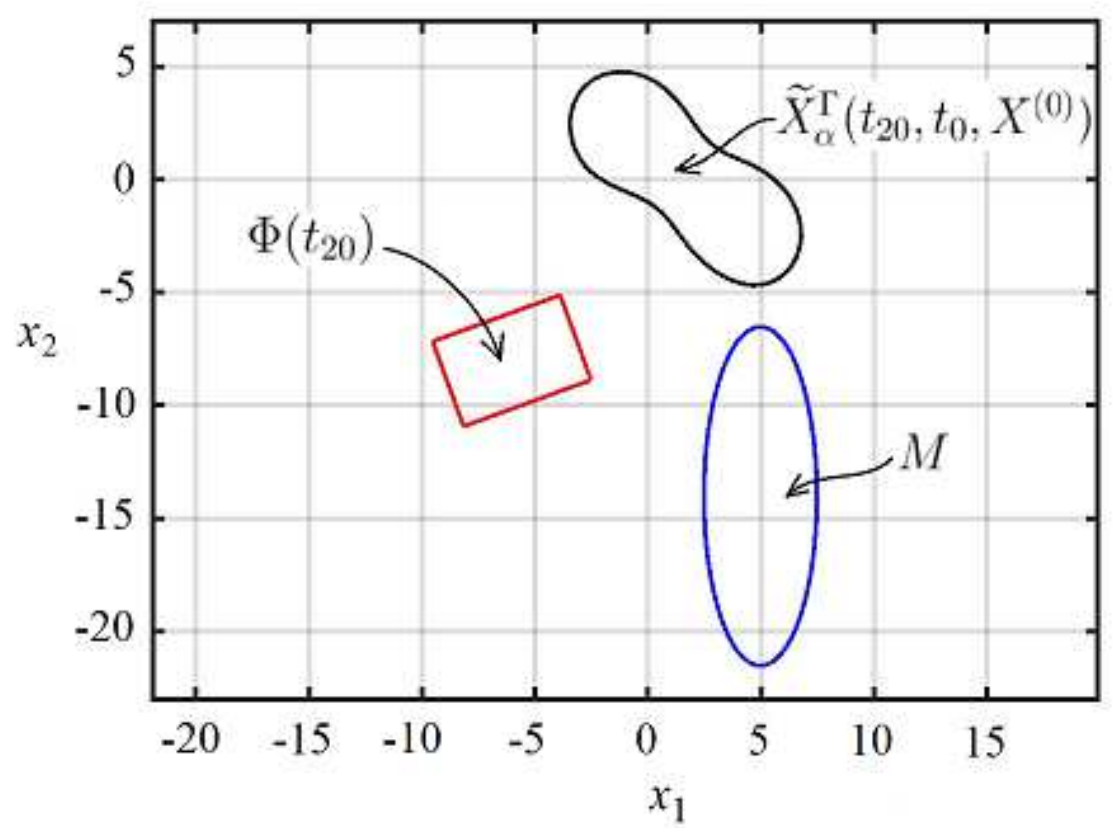

Figure 16. The sets $M, \Phi\left(t_{i}\right)$, and $\widetilde{X}_{\alpha}^{\Gamma}\left(t_{i}, t_{0}, X^{(0)}\right)$ for $\alpha_{1}^{*}=0.8, \alpha_{2}^{*}=0.33333$, and $t_{i}=0.2$. 


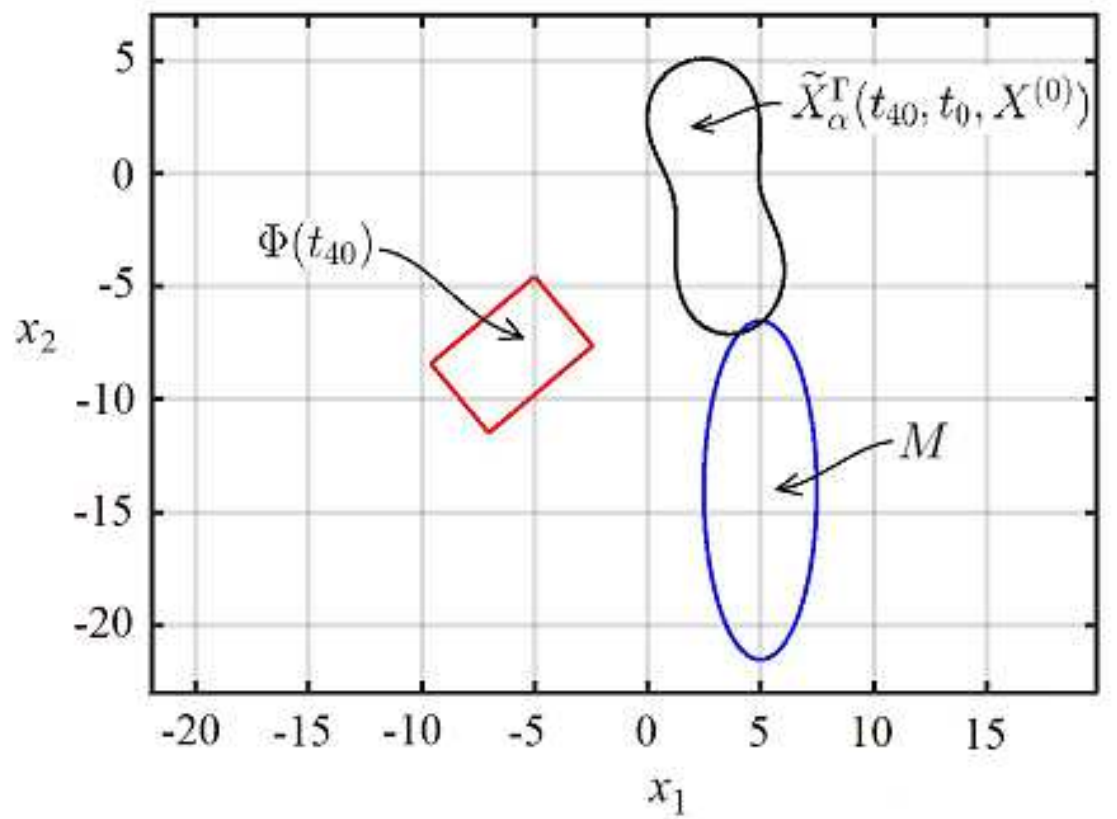

Figure 17. The sets $M, \Phi\left(t_{i}\right)$, and $\widetilde{X}_{\alpha}^{\Gamma}\left(t_{i}, t_{0}, X^{(0)}\right)$ for $\alpha_{1}^{*}=0.8, \alpha_{2}^{*}=0.33333$, and $t_{i}=0.4$.

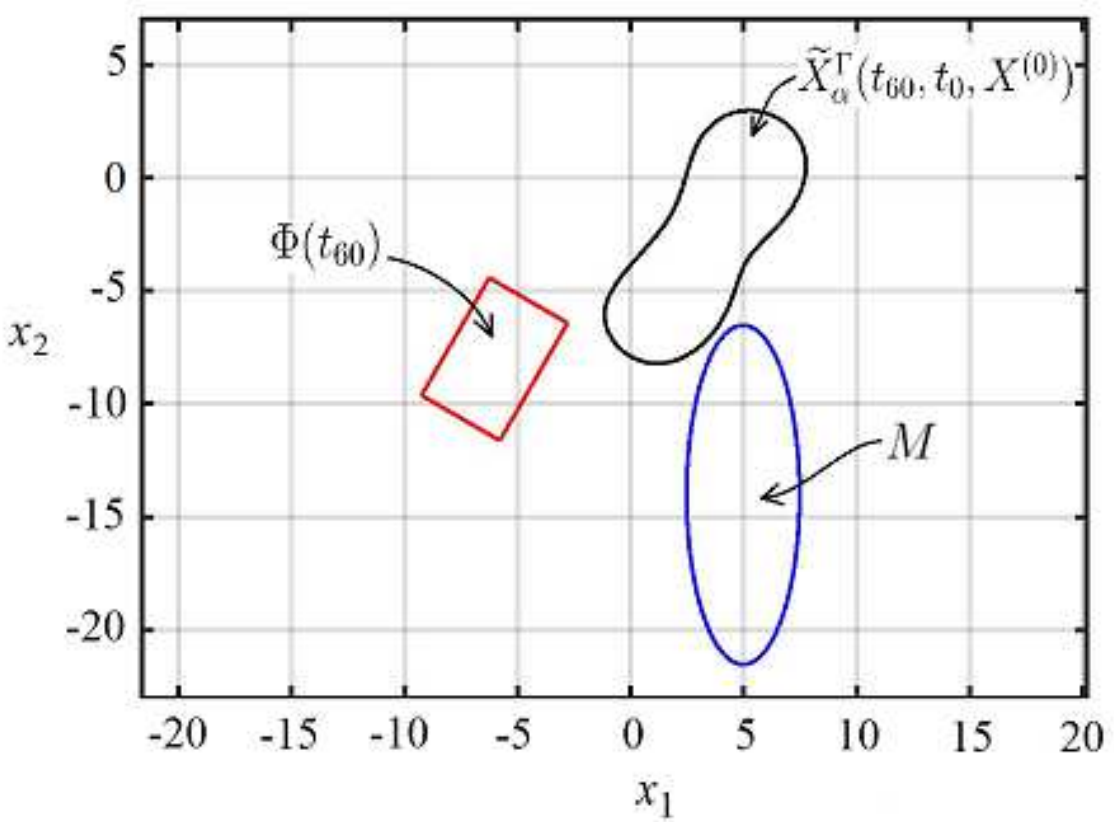

Figure 18. The sets $M, \Phi\left(t_{i}\right)$, and $\widetilde{X}_{\alpha}^{\Gamma}\left(t_{i}, t_{0}, X^{(0)}\right)$ for $\alpha_{1}^{*}=0.8, \alpha_{2}^{*}=0.33333$, and $t_{i}=0.6$. 


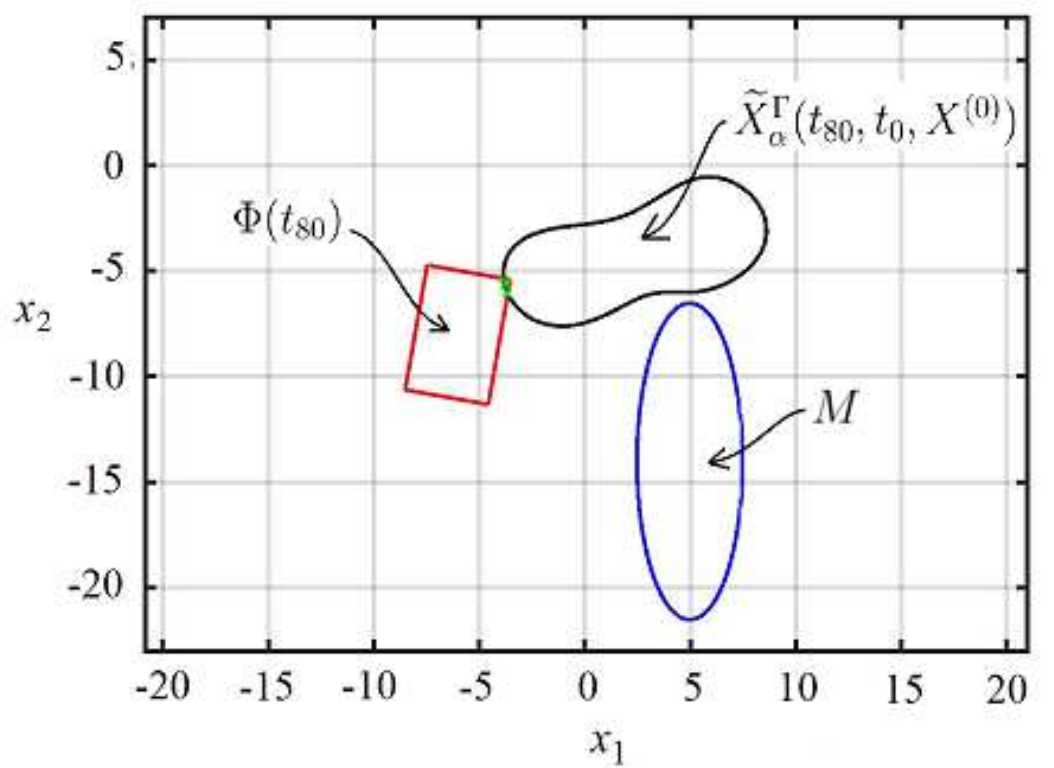

Figure 19. The sets $M, \Phi\left(t_{i}\right)$, and $\widetilde{X}_{\alpha}^{\Gamma}\left(t_{i}, t_{0}, X^{(0)}\right)$ for $\alpha_{1}^{*}=0.8, \alpha_{2}^{*}=0.33333$, and $t_{i}=0.8$.

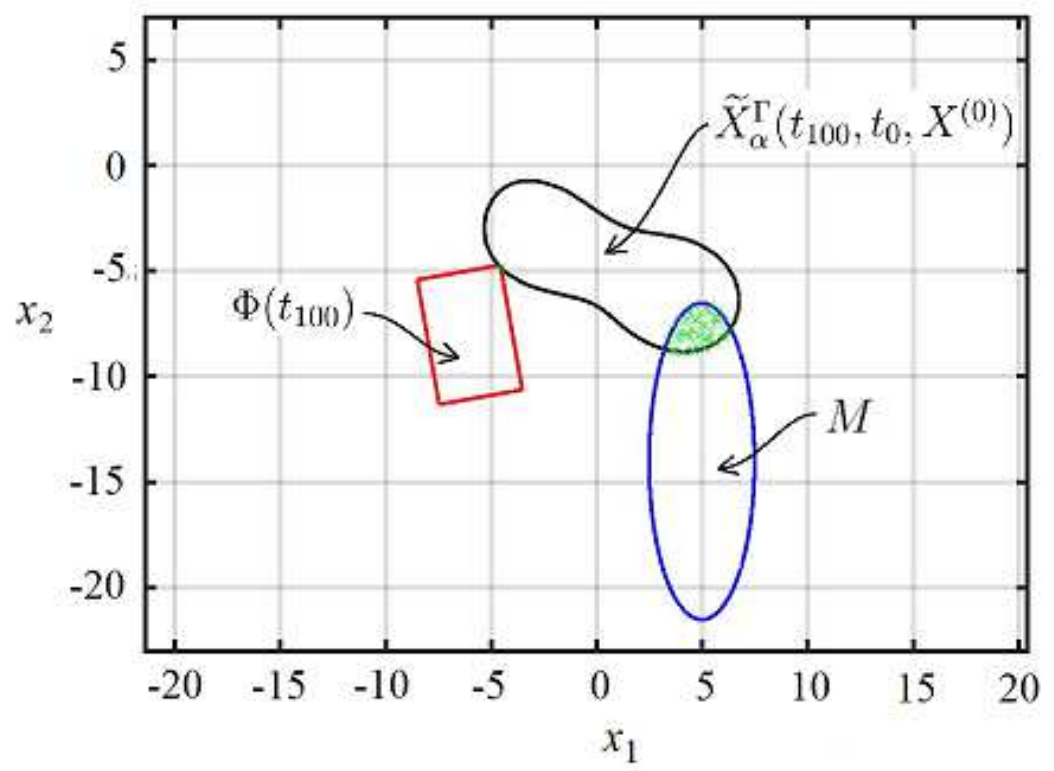

Figure 20. The sets $M, \Phi\left(t_{i}\right)$, and $\widetilde{X}_{\alpha}^{\Gamma}\left(t_{i}, t_{0}, X^{(0)}\right)$ for $\alpha_{1}^{*}=0.8, \alpha_{2}^{*}=0.33333$, and $t_{i}=1$. 
$\underline{\text { Variant } 3}$

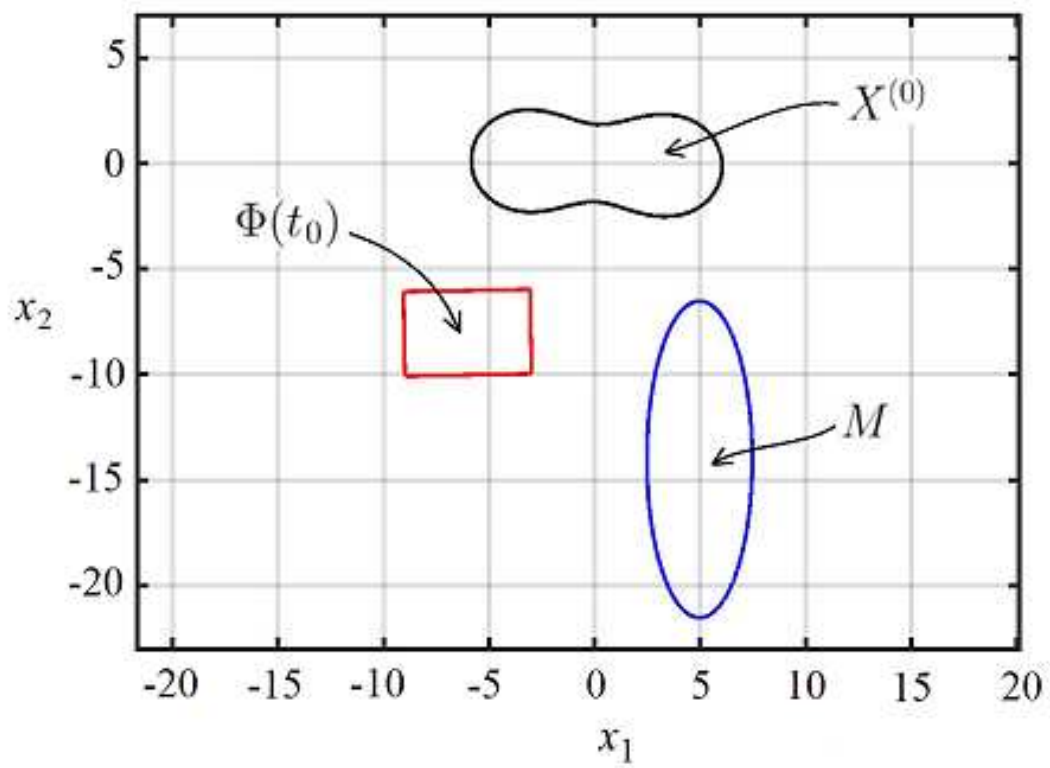

Figure 21. The sets $M, \Phi\left(t_{i}\right)$, and $\widetilde{X}_{\alpha}^{\Gamma}\left(t_{i}, t_{0}, X^{(0)}\right)$ for $\alpha_{1}^{*}=1.2667, \alpha_{2}^{*}=0.33333$, and $t_{i}=0$.

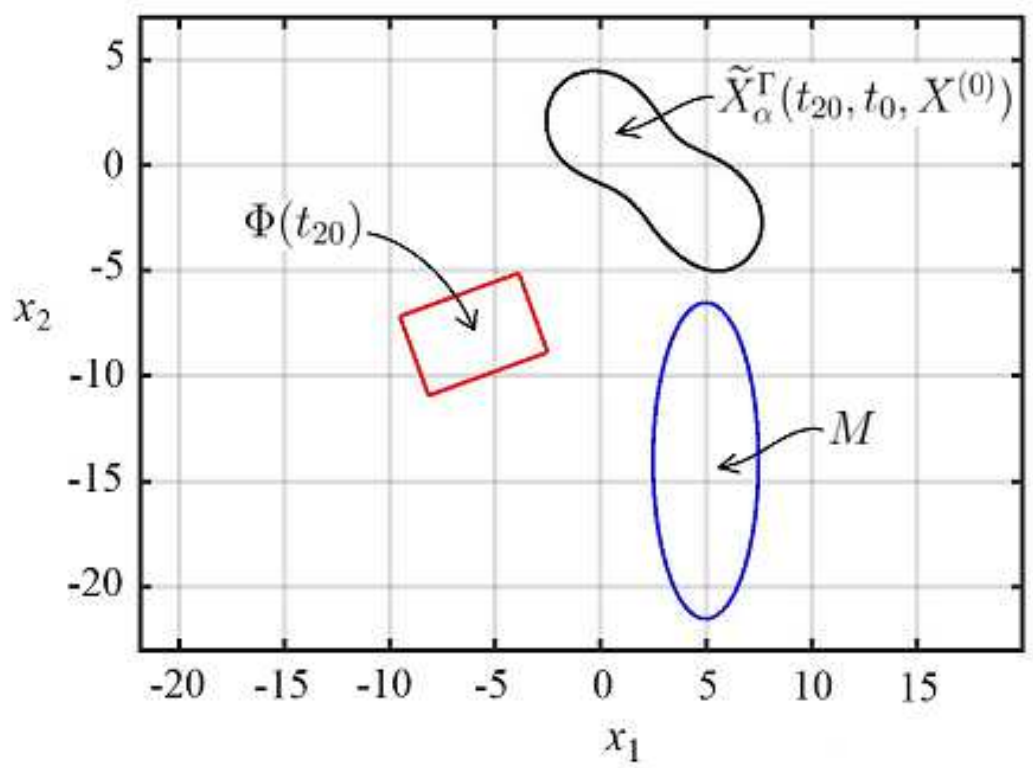

Figure 22. The sets $M, \Phi\left(t_{i}\right)$, and $\widetilde{X}_{\alpha}^{\Gamma}\left(t_{i}, t_{0}, X^{(0)}\right)$ for $\alpha_{1}^{*}=1.2667, \alpha_{2}^{*}=0.33333$, and $t_{i}=0.2$. 


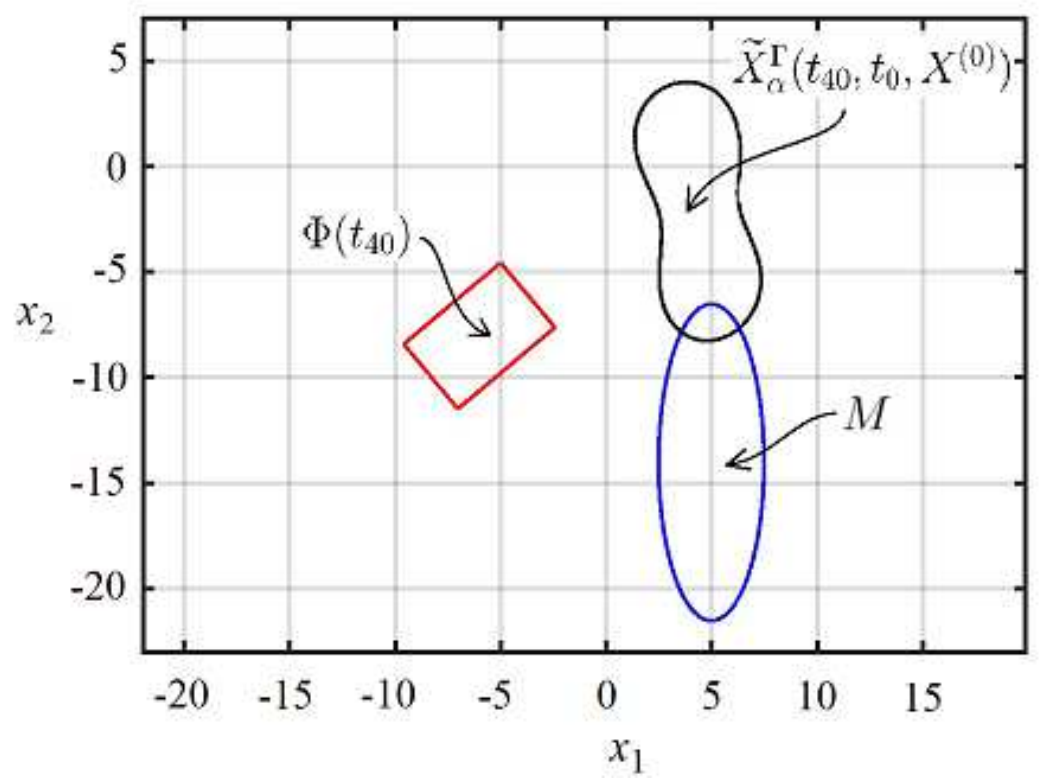

Figure 23. The sets $M, \Phi\left(t_{i}\right)$, and $\widetilde{X}_{\alpha}^{\Gamma}\left(t_{i}, t_{0}, X^{(0)}\right)$ for $\alpha_{1}^{*}=1.2667, \alpha_{2}^{*}=0.33333$, and $t_{i}=0.4$.

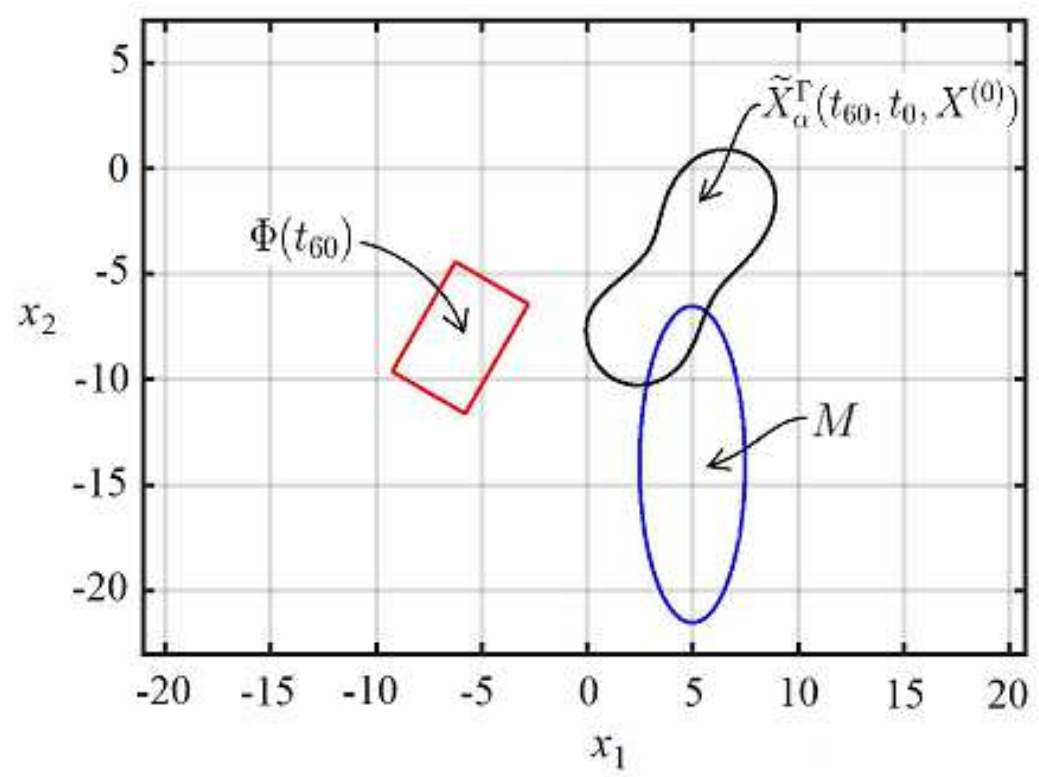

Figure 24. The sets $M, \Phi\left(t_{i}\right)$, and $\widetilde{X}_{\alpha}^{\Gamma}\left(t_{i}, t_{0}, X^{(0)}\right)$ for $\alpha_{1}^{*}=1.2667, \alpha_{2}^{*}=0.33333$, and $t_{i}=0.6$. 


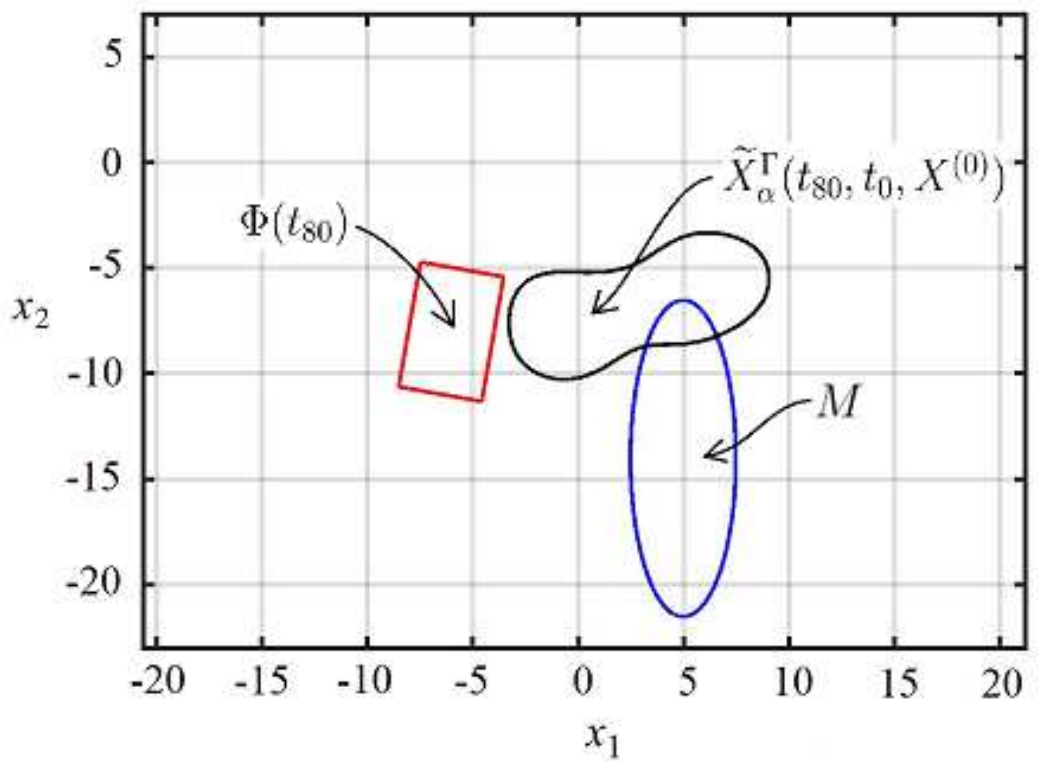

Figure 25. The sets $M, \Phi\left(t_{i}\right)$, and $\widetilde{X}_{\alpha}^{\Gamma}\left(t_{i}, t_{0}, X^{(0)}\right)$ for $\alpha_{1}^{*}=1.2667, \alpha_{2}^{*}=0.33333$, and $t_{i}=0.8$.

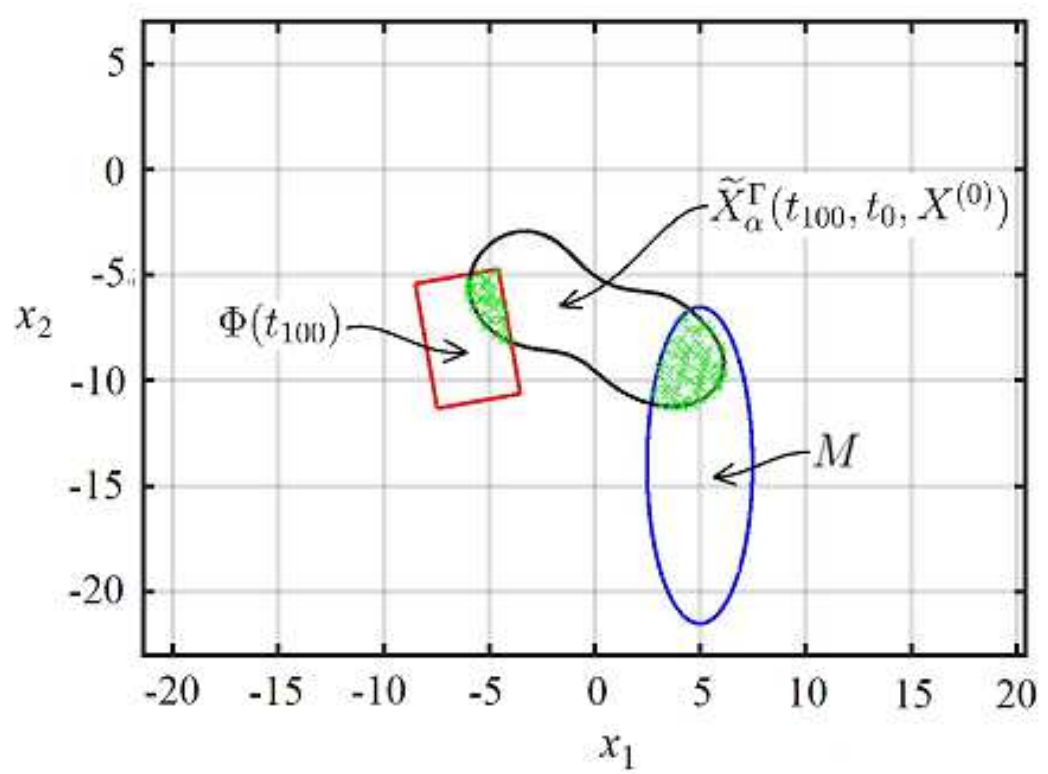

Figure 26. The sets $M, \Phi\left(t_{i}\right)$, and $\widetilde{X}_{\alpha}^{\Gamma}\left(t_{i}, t_{0}, X^{(0)}\right)$ for $\alpha_{1}^{*}=1.2667, \alpha_{2}^{*}=0.33333$, and $t_{i}=1$. 


\section{REFERENCES}

1. Anan'evskii I. M. Control of a nonlinear vibratory system of the fourth order with unknown parameters. Autom. Remote Control, 2001. Vol. 62, No. 3. P. 343-355. DOI: 10.1023/A:1002832924913

2. Anan'evskii I. M. Control synthesis for linear systems by methods of stability theory of motion. Differential Equations, 2003. Vol. 39, No. 1. P. 1-10. DOI: 10.1023/A:1025170521270

3. Beznos A. V., Grishin A. A., Lensky A. V., Okhotsimsky D. E., Formalsky A. M. Pendulum control using a flywheel. In: Spetspraktikum po teoreticheskoi i prikladnoi mehanike [Special workshop on theoretical and applied mechanics]. V.V. Aleksandrov, Yu.V. Bolotov (eds.). Moscow: MSU Press, 2019. P. 170-195.

4. Bogachev V.I., Smoljanov O.G. Deistvitel'nyi i funktsional'nyi analiz: universitetskii kurs [Real and Functional Analysis: University Course]. Moscow-Izhevsk: Research Center "Regular and Chaotic Dynamics", Institute for Computer Research, 2009. 724 p. (in Russian)

5. Chernousko F. L. State Estimation for Dynamic Systems. CRC Press: Boca Raton, 1994. 320 p.

6. Chernousko F. L., Melikyan A. A. Igrovye zadachi upravlenija i poiska [Game Control and Search Problems]. Moscow: Nauka, 1978. 270 p. (in Russian)

7. Ershov A. A., Ushakov V.N. An approach problem for a control system with an unknown parameter. Sb. Math., 2017. Vol. 208. No. 9. P. 1312-1352. DOI: 10.1070/SM8761

8. Filippova T.F. Construction of set-valued estimates of reachable sets for some nonlinear dynamical systems with impulsive control. Proc. Steklov Inst. Math., 2010. Vol. 269, Suppl. 1. P. S95-S102. DOI: $10.1134 /$ S008154381006009X

9. Gusev M.I. Estimates of reachable sets of multidimensional control systems with nonlinear interconnections. Proc. Steklov Inst. Math., 2010. Vol. 269, Suppl. 1. P. S134-S146. DOI: $10.1134 /$ S008154381006012X

10. Krasovsky N. N. Upravlenie dinamicheskoi sistemoi: Zadacha o minimume garantirovannogo rezul'tata [Control of a Dynamical System: Problem on the Minimum of Guaranteed Result]. Moscow: Nauka, 1985. 520 p. (in Russian)

11. Krasovsky N.N., Subbotin A.I. Pozitsionnye differentsial'nye igry [Positional Differential Games]. Moscow: Fizmatlit, 1974. 456 p. (in Russian)

12. Kurzhansky A. B. Izbrannye trudy [Selected Works]. Moscow: MSU Press, 2009. 756 p. (in Russian)

13. Kurzhanski A. B., Valyi I. Ellipsoidal Calculus for Estimation and Control. Systems Control Found. Appl. Basel: Birkhäuser, 1997. 321 p.

14. Lee E. B., Markus L. Foundation of Optimal Control Theory. New York-London-Sydney: John Wiley \& Sons, 1967. 576 p.

15. Leichtweiß K. Konvexe Mengen. Hochschultext. Berlin: Springer-Verlag, 1979. 330 p. (in German)

16. Lempio F., Veliov V. M. Discrete approximation of differential inclusions. Bayreuth. Math. Schr., 1998. Vol. 54. P. 149-232.

17. Nikol'skii M.S. On the approximation of the reachable set of a differential inclusion. Vestnik Moskov. Univ. Ser. XV Vychisl. Mat. Kibernet, 1987. No. 4. P. 31-34.

18. Nikol'skii M.S. An inner estimate of the attainability set of Brockett's nonlinear integrator. Differential Equations, 2000. Vol. 36, No. 11. P. 1647-1651. DOI: 10.1007/BF02757366

19. Polyak B. T., Khlebnikov M. V., Shcherbakov P.S. Upravleniye lineynymi sistemami pri vneshnih vozmushcheniyah: Tehnika lineynyh matrichnyh neravenstv [Control of linear systems under external disturbances: Technique of linear matrix inequalities]. Moscow: LENAND, 2014. 560 p. (in Russian)

20. Ushakov V.N., Matviychuk A.R., Ushakov A.V. Approximations of attainability sets and of integral funnels of differential inclusions. Vestn. Udmurtsk. Univ. Mat. Mekh. Komp. Nauki, 2011. No. 4. P. 23-39. (in Russian) URL: http://vst.ics.org.ru/journal/article/1816/

21. Vdovin S. A., Taras'yev A. M., Ushakov V. N. Construction of the attainability set of a Brockett integrator. J. Appl. Math. Mech., 2004. Vol. 68, No. 5. P. 631-646. DOI: 10.1016/j.jappmathmech.2004.09.001 